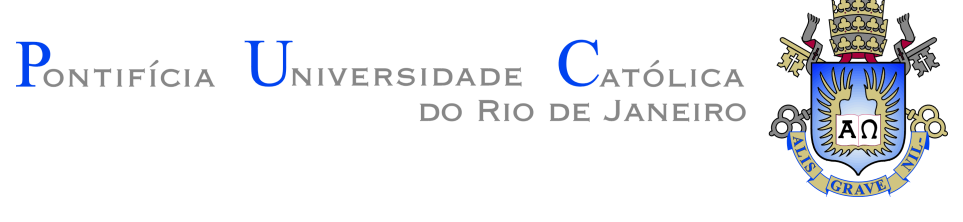

André Xavier Leitão

Topology Optimization of Geometrically Nonlinear Structures Based on an Energy Interpolation Scheme

Dissertation presented to the Programa de Pós-Graduação em Engenharia Mecânica of PUC-Rio in partial fulfillment of the requirements for the degree of Mestre em Engenharia Mecânica.

Advisor: Prof. Anderson Pereira

Rio de Janeiro

May 2019 


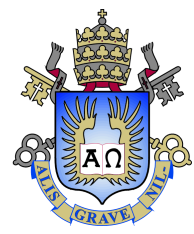

André Xavier Leitão

\section{Topology Optimization of Geometrically Nonlinear Structures Based on an Energy Interpolation Scheme}

Dissertation presented to the Programa de Pós-Graduação em Engenharia Mecânica of PUC-Rio in partial fulfillment of the requirements for the degree of Mestre em Engenharia Mecânica. Approved by the Examination Committee.

Prof. Anderson Pereira

Advisor

Departamento de Engenharia Mecânica - PUC-Rio

Prof. Ivan Fábio Mota de Menezes

Departamento de Engenharia Mecânica - PUC-Rio

Prof. Francisco José da Cunha Pires Soeiro Universidade do Estado do Rio de Janeiro - UERJ 
All rights reserved.

\section{André Xavier Leitão}

The author holds a bachelor's degree in Mechanical Engineering from Pontifícia Universidade Católica do Rio de Janeiro, PUC-Rio, in 2015.

Bibliographic data

Leitão, André Xavier

Topology Optimization of Geometrically Nonlinear Structures Based on an Energy Interpolation Scheme / André Xavier Leitão; advisor: Anderson Pereira - 2019.

104 f.: il. color.; $30 \mathrm{~cm}$

Dissertação de Mestrado - Pontifícia Universidade Católica do Rio de Janeiro, Departamento de Engenharia Mecânica, 2019.

Inclui bibliografia

1. Engenharia Mecânica - Teses. Otimização topológica; Não-linearidade geométrica; Elementos finitos; Método de interpolação; Instabilidade numérica. I. Pereira, Anderson. II. Pontifícia Universidade Católica do Rio de Janeiro. Departamento de Engenharia Mecânica. III. Título. 
To my family, for their support and encouragement. And to my grandparents Lourdinha and Francisco (in memoriam). 


\section{Acknowledgments}

First of all, I thank God for the opportunity to attend this Master's program, allowing me to grow not only professionally, but also as a person. I am grateful for all the help that came from the spiritual plane, which in difficult times gave me the courage and the strength to move on.

Here on Earth, I thanks Professor Ivan Menezes for presenting the basic concepts and main ideas of optimization and my advisor, Professor Anderson Pereira, who was always present during my thesis. Several times we talked, sometimes late at night, and I was able to clarify ideas, ask questions and... learn. I am grateful for the time attending classes, where I was able to develop my knowledge in areas of interest and be in contact with people who stimulate study and academic research, which I cite Professors Carlos Alberto de Almeida, José Luiz de França Freire and Jaime Tupiassú Pinho de Castro. I also appreciate the staff of the Mechanical department (DEM) for the assistance and support to provide the necessary conditions to the Laboratório de Computação Avançada (LCA), so I could work on this thesis.

I have to mention my friends who supported me in this thesis, whether through a council, a friendly word or simply listening to my concerns, especially, Edson Sabino, Eduardo Cota, João Carlos Virgolino, Juan Hidalgo, Miguel Suárez, Rodrigo Bianchi, Thiago Almeida and Waldy Zuniga, besides others that I lived during the Master's.

My family plays an important role in my life, and without them, everything will be harder. My mom Anna and dad José are always there assisting me. My brother Bruno probably has its ears burning, after listening to my complaints and fears several times during this work. But he is always there for me. My grandma, Daisy, is everything to me. She did so much for me, since I was born, that some of them I can not even remember. During this master's, it was not different and I will always be thankful for everything she has done to me.

For last, I would like to express my gratitude for the financial support provided by CNPq (Conselho Nacional de Desenvolvimento Científico e Tecnológico), especially in times of economic and political uncertainties. 


\section{Abstract}

Leitão, André Xavier; Pereira, Anderson (Advisor). Topology Optimization of Geometrically Nonlinear Structures Based on an Energy Interpolation Scheme. Rio de Janeiro, 2019. 104p. Dissertação de Mestrado - Departamento de Engenharia Mecânica, Pontifícia Universidade Católica do Rio de Janeiro.

In many engineering problems, e.g., design of flexible biomedical prostheses or energy absorption devices, structures undergo large displacements. In those problems, the structural response must take into account the geometric nonlinearity. However, topology optimization algorithms regarding nonlinearities, and based on the finite element method, typically suffer from numerical instabilities caused by excessive distortions of low-density regions within the design domain. In particular, the stiffness matrix may be no longer positive definite, which can jeopardize the convergence of the optimization process. This thesis aims to study an interpolation scheme between linear and nonlinear finite element formultation to alleviate this convergence issue. At each step of the optimization, the nonlinear state equation is solved by the Newton-Raphson procedure to determine the equilibrium configuration. Making use of the gradient information computed from the adjoint method, the Method of Moving Asymptotes is employed to update the design variables. Through several benchmark problems considering large displacements, it is demonstrated the effectiveness and efficiency of this interpolation scheme. More specifically, the optimized designs are in agreement with those obtained in the literature and exhibit correct load-level dependence. The investigated interpolation scheme plays a crucial role in the solution of nonlinear problems with high load levels, allowing the optimization routine to converge and to obtain the optimal material arrangement.

\section{Keywords}

Topology Optimization; End-compliance Minimization; Geometrical Nonlinearity; Nonlinear Solution; Finite Elements; Interpolation Scheme; Numerical Instabilities; Low-Density Elements; Sensitivity Analysis. 


\section{Resumo}

Leitão, André Xavier; Pereira, Anderson (Orientador). Otimização Topológica de Estruturas Geometricamente Nãolineares Baseada em um Esquema de Interpolação de Energia. Rio de Janeiro, 2019. 104p. Dissertação de Mestrado — Departamento de Engenharia Mecânica, Pontifícia Universidade Católica do Rio de Janeiro.

Em muitos problemas de engenharia, e.g., no projeto de próteses biomédicas flexíveis ou em dispositivos de absorção de energia, estruturas sofrem grandes deslocamentos. Nestes casos, a não linearidade geométrica deve ser levada em conta na resposta estrutural. Contudo, algoritmos de otimização topológica considerando não linearidades, e modelados segundo o método de elementos finitos, sofrem instabilidades numéricas causadas por distorções excessivas nas regiões de baixa densidade dentro do domínio de projeto. Em particular, a matriz de rigidez pode não ser positiva definida comprometendo a convergência do processo de otimização. Esta dissertação visa estudar um esquema de interpolação entre as formulações lineares e não lineares de elementos finitos para aliviar tais distorções. Em cada etapa da otimização, para determinar a configuração de equilíbrio, o sistema de equações não-lineares é resolvido pelo procedimento de Newton-Raphson. Utilizando-se das informações dos gradientes calculadas através do método adjunto, o Método das Assíntotas Móveis é empregado para atualizar as variáveis de projeto. Por meio de problemas de referência considerando grandes deslocamentos, são demonstradas a eficácia e a eficiência deste esquema de interpolação. Mais especificamente, as topologias otimizadas estão de acordo com aquelas obtidas na literatura e exibem a dependência esperada em relação ao nível de carga. O esquema de interpolação em estudo desempenha papel crucial na solução de problemas não lineares em níveis elevados de carga, permitindo que a rotina de otimização convirja e se obtenha a distribuição de material ótima.

\section{Palavras-chave}

Otimização Topológica; Minimização da Flexibilidade; Não-linearidade Geométrica; Solução Não-linear; Elementos Finitos; Método de Interpolação; Instabilidade Numérica; Elementos de Baixa Densidade; Análise de Sensibilidade. 


\section{Contents}

1 Introduction $\quad 20$

1.1 Motivation 22

1.2 Objective 24

1.3 Literature Review 24

$\begin{array}{lll}1.4 & \text { Outline } & 28\end{array}$

2 A Brief Review on the Finite Element Method 29

$\begin{array}{lll}2.1 \text { Introduction } & 29\end{array}$

2.2 Mechanics of Deformable Bodies 29

2.2.1 Strain Measures 31

2.2.2 Stress Measures 33

2.3 Finite Element Formulation in Nonlinear Problems 34

2.3.1 Linearization of Incremental Equations 36

2.3.2 Element Isoparametric Formulation 37

2.3.2.1 Quadrilateral Elements 38

2.3.3 Matrix Form 41

2.4 Numerical Integration 43

3 Topology Optimization $\quad 45$

3.1 Introduction 45

3.2 Material Representation $\quad 46$

3.2.1 Solid Isotropic Material with Penalization (SIMP) 46

3.2.2 Continuation Scheme 48

3.3 Complications 48

$\begin{array}{lll}\text { 3.3.1 Gray Scale } & 49\end{array}$

3.3.2 Checkerboard Pattern 49

3.3.3 Mesh-Dependency and Non-uniqueness of Solution 50

3.4 Regularization Techniques $\quad 51$

3.4.1 Restriction Methods $\quad 52$

3.4.1.1 Perimeter Control $\quad 52$

3.4.1.2 Local Gradient Constraint $\quad 52$

3.4.2 Linear Filter $\quad 52$

3.4.3 Nonlinear Projection Techniques $\quad 55$

3.4.3.1 Exponential Projection Function $\quad 55$

3.4.3.2 Hyperbolic Tangent (tanh) Projection Function 56

4 Topology Optimization Under Geometric Nonlinearity 58

$\begin{array}{lll}4.1 & \text { Introduction } & 58\end{array}$

4.2 Optimization Problem 58

4.3 Modeling of Void Regions $\quad 59$

4.3.1 Tangent Stiffness Matrices and Internal Force Vectors 61

4.3.2 C-shaped Benchmark Problem 62

4.4 Sensitivity Analysis of the Objective 65

4.5 Nonlinear Solution Schemes 67 
$\begin{array}{ll}\text { 4.5.1 Unified Library } & 70\end{array}$

4.5.1.1 Load Control Method $\quad 72$

4.5.1.2 Arc-Length Method 73

4.5.1.3 Generalized Displacement Control Method 76

$\begin{array}{lll}4.6 & \text { Flowchart } & 78\end{array}$

5 Results $\quad 80$

$\begin{array}{lll}5.1 & \text { Introduction } & 80\end{array}$

5.2 Cantilever Beam $\quad 80$

5.2.1 Comparison with Literature 83

5.3 Clamped Beam $\quad 85$

5.3.1 Comparison with Literature $\quad 86$

6 Conclusions $\quad 88$

6.1 Suggestions for Future Works $\quad 89$

$\begin{array}{lr}\text { Bibliography } & 91\end{array}$

A Verification of the Nonlinear Solution Scheme $\quad 97$

B Validation of Sensitivity of Objective Function 101

C Articles from this Thesis 103 


\section{List of Figures}

1.1 Optimization methods (Bendsøe \& Sigmund, 2003). 20

1.2 Illustrative scheme for a topology optimization project (adapted from VTT subproject (SIMPRO)).

1.3 Optimization process of a rib structure in the front part of the wing of Airbus A380: (a) design domain; (b) optimized layout; (c) actual structure; (d) manufactured wing structure (Bendsøe \& Sigmund, 2007).

1.4 Body support structure to help a disabled person lift his arm (adapted from Holtzer (2017)).

2.1 Schematic motion and deformation of a solid in space.

2.2 Mapping of a square in local coordinate system to an arbitrary straight-sided quadrilateral in global coordinate system.

2.3 Representation of a 4-to-9-node quadrilater
2.4 Gauss integration points of Q4 element.

3.1 Representation of a topology optimization problem of design domain $\Omega$ (adapted from Talischi et al. (2012b)).

3.2 Influence of different penalization exponents in SIMP method.

3.3 Schematization of continuation method (adapted from Lahuerta (2012)).

3.4 The MBB beam with gray scale. Result obtained without continuation or any penalization method.

3.5 Checkerboard pattern demonstrated in the MBB beam.

3.6 Dependency of the optimal topology on mesh-refinement for the MBB beam for different mesh discretizations (adapted from Bends $\varnothing$ e \& Sigmund (2003)).

3.7 Two-dimensional linear density filter: (a) application in a regular quadrilateral element mesh; (b) conical form alike to a birthday party hat.

3.8 Regularized exponential function for several magnitudes of $\beta_{1}$. $\quad 56$

3.9 Hyperbolic tangent function for several magnitudes of $\beta_{2}$. $\quad 57$

4.1 C-shape. $\quad 62$

4.2 C-shape geometry when modeling only solid elements. 63

4.3 C-shape linear analysis when modeling solid (light blue) and void (white) elements in a fictitious domain representation.

4.4 C-shape nonlinear analysis. Deformation and displacement field for the indicated cases.

$\begin{array}{lll}4.5 & \text { Representative equilibrium path. } & 67\end{array}$

4.6 Incremental-iterative procedure. 68

$\begin{array}{lll}4.7 & \text { Load control method. } & 72\end{array}$

$\begin{array}{lll}4.8 & \text { Arc-length method. } & 74\end{array}$

4.9 Flowchart for geometric nonlinear topology optimization. $\quad 79$ 
5.1 Cantilever beam domain. $\quad 80$

5.2 Optimal topologies and corresponded compliance values for the indicated loads obtained using the interpolation scheme.

5.3 Element's behavior at the end of the optimization process for an applied force of $300 \mathrm{kN}$.

5.4 Clamped beam domain.

A.1 Lee frame. "DETAIL A" shows the point where the load $\boldsymbol{q}$ is applied (point B).

A.2 Lee frame equilibrium path: responses for the implemented code and the APDL for Q4 elements.

A.3 Lee frame equilibrium path: responses for the implemented code and the APDL for Q8 elements.

A.4 Deformation at the points indicated in Figure A.3: (a) linear regime; (b) load limit point in traction; (c) first displacement limit point; (d) second displacement limit point; (e) load limit point in compression; (f) final configuration.

B.1 Tested beam: design domain, boundary conditions, load and element numbering.

C.1 Article published in the X CONEM: title, abstract and keywords. 103

C.2 Article accepted at the 25th COBEM: title, abstract and keywords. 104 


\section{List of Tables}

2.1 Interpolation functions for quadrilateral elements (adapted from Bathe (2014)).

2.2 Gauss integration over Q4 element (adapted from Bathe (2014)). 44

4.1 Versions of arc-length method. 74

4.2 Input data for the Newton-Raphson procedure. 78

5.1 Cantilever beam optimal topologies and compliance values for several load levels considering linear and nonlinear analyses.

5.2 Comparison of optimized topologies for the cantilever beam for three different loads.

5.3 Clamped beam optimal topologies and compliance values for two load levels considering linear analysis and the interpolation scheme. 86

5.4 Comparison of optimized topologies for the clamped beam for a load of $230 \mathrm{kN}$.

B.1 Comparison between objective function derivatives calculated analytically and via finite differences for a load of $15 \mathrm{kN}$.

B.2 Comparison between objective function derivatives calculated analytically and via finite differences for a load of $100 \mathrm{kN}$. 


\section{List of Abbreviations}

APDL - ANSYS ${ }^{\circledR}$ Parametric Design Language

ALM - Arc-Length Method

FEM - Finite Element Method

GDCM - Generalized Displacement Control Method

GSP - Generalized Stiffness Parameter

LBB - Ladyzenskaja-Babuska-Brezzi (condition)

LCM - Load Control Method

mSVK - modified St. Venant-Kirchoff (material model)

MBB - Messerschmitt-Bölkow-Blohm

MMA - Method of Moving Asymptotes

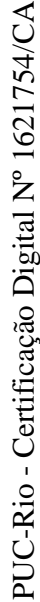

$\mathrm{nH}$ - neo-Hookean (material model)

NRP - Newton-Raphson Procedure

OC - Optimality Criteria

Q4 - 4-node quadrilateral element

Q8 - 8-node quadrilateral element

SIMP - Solid Isotropic Material with Penalization

SVK - St. Venant-Kirchoff (material model)

TOM - Topology Optimization Method

TL - Total Lagrangian (formulation)

UL - Updated Lagrangian (formulation)

VEM - Virtual Element Method 


\section{List of Symbols}

\section{Math Operators}

$\nabla \quad$ gradient operator in the current configuration of the body

$\nabla_{0} \quad$ gradient operator in the reference configuration of the body

dot product

: contraction tensor operator

det determinant

sign signal of a variable

$\backslash$ relative complement of two sets

$\cap \quad$ assembly operator

$D(\bullet) \quad$ directional derivative

\section{Continuum Mechanics}

$\boldsymbol{x}$

position of a continuum particle (current configuration)

$\boldsymbol{X}$ position of a continuum particle (reference configuration)

$\boldsymbol{u} \quad$ displacement vector

$\chi \quad$ deformation mapping

$\boldsymbol{F} \quad$ deformation gradient tensor

$J \quad$ Jacobian of transformation: $J=\operatorname{det}(\boldsymbol{F})$

I identity matrix

C right Cauchy-Green deformation tensor

$\boldsymbol{b} \quad$ left Cauchy-Green deformation tensor

$\boldsymbol{E} \quad$ Green-Lagrangian strain tensor

$e$

Almansi-Eulerian strain tensor

$\delta \quad$ Kronecker delta

$\sigma$

Cauchy stress tensor

$\boldsymbol{P} \quad$ 1st Piola-Kirchhoff stress tensor

$\boldsymbol{S} \quad$ 2nd Piola-Kirchhoff stress tensor

$\boldsymbol{\tau} \quad$ Kirchhoff stress tensor 


\section{Finite Element Analysis and Numerical Integration}

(仑)

$(\bullet)$

(•)

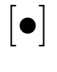

$\varrho$

$\boldsymbol{f}^{B}$

$\boldsymbol{f}_{0}^{B}$

$t$

$t_{0}$

${ }^{0} \Omega$

${ }^{t} \Omega$

${ }^{0} \Gamma$

${ }^{t} \Gamma$

$\boldsymbol{J}_{0}$

$\mathcal{C}^{0}$

$D^{0}$

K

$\boldsymbol{K}^{L}$

$\boldsymbol{K}^{D}$

$\boldsymbol{K}^{S}$

$\boldsymbol{B}_{(\bullet)}$

$\left(\xi_{1}, \xi_{2}\right)$

$h$

$n$

$N G$

$\omega$ vector representation of matrices

virtual quantity of tensor

rate of tensors

direction of linearization

magnitude of linearization

vector of force per unit deformed volume

vector of force per unit undeformed volume

traction vector written in spatial coordinates

traction vector written in material coordinates

reference configuration of a body

current configuration of a body

region where $\boldsymbol{t}$ is applied

region where $\boldsymbol{t}_{0}$ is applied

Jacobian matrix

Elasticity tensor (without any penalization)

matrix representation of $\mathcal{C}^{0}$

tangent stiffness matrix

linear stiffness matrix

"initial displacement" stiffness matrix

initial stress stiffness matrix

strain-displacement matrices

isoparametric coordinate for two-dimensional problems

shape (or Lagrangian interpolation) function

nodal points

number of integration points in the Gauss quadrature

weight of integration points 


\section{Material Properties and Geometry}

$\begin{array}{ll}L & \text { length } \\ H & \text { height } \\ d & \text { depth (thickness) } \\ E_{0} & \text { Young's modulus } \\ \nu_{0} & \text { Poisson's coefficient }\end{array}$

\section{Topology Optimization}

$\Omega \quad$ (fixed) design domain

$\partial \Omega \quad$ boundaries of $\Omega$

$\Omega_{s} \quad$ region occupied by solid (or structural) elements in $\Omega$

$\Omega_{v} \quad$ region occupied by void elements in $\Omega$

$N_{e} \quad$ number of elements in $\Omega$

Ersatz parameter

density

$\boldsymbol{z} \quad$ design variable

$\boldsymbol{y} \quad$ "filtered" design variable

$\tilde{\boldsymbol{x}} \quad(\mathrm{SIMP})$ penalized design variable

$p \quad$ penalization exponent

$\Delta p \quad$ increment of penalization exponent in the continuation scheme

$\bar{z} \quad$ location of the design variable

$w_{i} \quad$ weight distribution function for the $i$ th design variable in the filtering

$d_{i} \quad$ distance from the $i$ th element

$r_{\text {min }} \quad$ filter radius

$\boldsymbol{M} \quad$ matrix of weighted values in the filtering

$g(\boldsymbol{y}) \quad$ nonlinear interpolation function

$\beta_{1} \quad$ projection parameter of the exponential projection function

$\beta_{2} \quad$ projection parameter of the tanh projection function

$\eta \quad$ threshold parameter of the tanh projection function

$\boldsymbol{\psi} \quad$ adjoint vector

$F_{c} \quad$ objective ("end-compliance") 


\section{Topology Optimization (continuation)}

$V_{0} \quad$ initial amount of volume

$V_{f} \quad$ final amount of volume

$V_{\text {frac }} \quad$ specified volume fraction

$\mathrm{CONV}$ nonlinear solution method convergence tolerance

TOL topology optimization convergence tolerance

Iter $_{\max }$ number of allowed iterations for each penalization exponent

\section{Interpolation Scheme}

$\phi \quad$ stored elastic energy density function

$\phi_{L} \quad$ stored elastic energy density function under small deformations

$\alpha \quad$ interpolation function sharpness parameter

$\bar{\rho} \quad$ interpolation function threshold parameter

interpolation factor of the eth element

$\boldsymbol{K}_{e}^{L} \quad$ element stiffness matrix under small deformations

$\boldsymbol{K}_{e}^{N L} \quad$ element stiffness matrix under large deformations

$\boldsymbol{f}_{e}^{L} \quad$ element internal force vector under small deformations

$\boldsymbol{f}_{e}^{N L} \quad$ element internal force vector under large deformations

\section{Nonlinear Solution Schemes - Unified Library}

notation for the incremental-iterative analysis: $s$ represents the incremental step and $i$ the iteration

$\boldsymbol{q} \quad$ applied force vector

$\boldsymbol{l} \quad$ external force vector

$f \quad$ internal force vector

$\boldsymbol{r} \quad$ residual or unbalanced force vector

${ }_{i}^{s} \Delta \boldsymbol{l} \quad$ incremental update external force vector

${ }_{i}^{s} \Delta \boldsymbol{u} \quad$ incremental update displacement vector

${ }_{i}^{s} \delta \boldsymbol{l} \quad$ iterative update external force vector

${ }_{i}^{s} \delta \boldsymbol{u} \quad$ iterative update displacement vector

${ }_{i}^{s} \delta \boldsymbol{u}_{l} \quad$ portion of ${ }_{i}^{s} \delta \boldsymbol{u}$ associated to $\boldsymbol{l}$

${ }_{i}^{s} \delta \boldsymbol{u}_{r} \quad$ portion of ${ }_{i}^{s} \delta \boldsymbol{u}$ associated to $\boldsymbol{r}$ 
Nonlinear Solution Schemes - Unified Library (continuation)

$\bar{l} \quad$ reference force vector

${ }_{i}^{s} \boldsymbol{a},{ }_{i}^{s} b,{ }_{i}^{s} c$ constraint equation control variables of unified library

${ }_{i}^{s} \delta \lambda \quad$ load factor or load parameter

$s \overline{\Delta \lambda} \quad$ prescribed initial load factor

${ }^{s} \overline{\Delta R} \quad$ prescribed initial arc-length

$\overline{\delta \lambda} \quad$ prescribed initial control factor

$\beta \quad$ non-negative real parameter defining the version of ALM 
The mind, once expanded to the dimensions of larger ideas, never returns to its original size.

Oliver Wendell Holmes, Sr. 


\section{Introduction}

Structural optimization can be divided into three categories: sizing, shape and topology. Concerning sizing optimization, the goal is to find the optimum value for a certain parameter, the design variable, typically the thickness of a plate or the cross section areas of a truss structure. The main feature of the sizing problem is that the design domain is known a priori and is fixed throughout the optimization process (Bendsøe \& Sigmund, 2003). In shape optimization, the boundaries are changed to achieve the optimum geometry. The domain is modified at each iteration and the connectivities remains the same. An application of shape optimization is on the reduction of stress concentration in equipments subjected to cyclic loading to predict crack initialization and propagation that eventually led to failure of the structure.

In topology optimization the number, location and shape of holes and their connectivities are determined during the optimization. The key is to find where to introduce and remove material according to the constraints of the problem. Different from the other methods, topology optimization can be used in conceptual phases to meet specific applications and conditions of a project. Figure 1.1 shows a typical example of each type of optimization method.

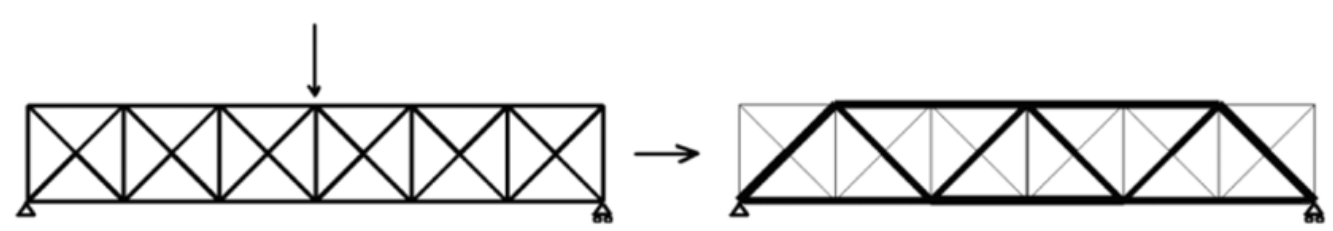

Sizing Optimization

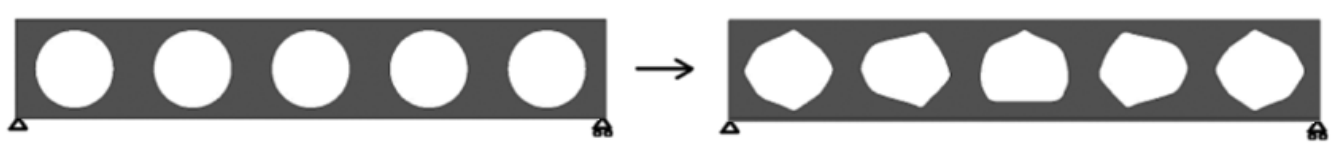

Shape Optimization

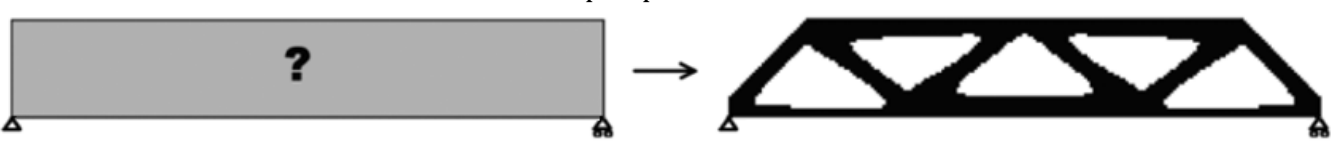

Topology Optimization

Figure 1.1: Optimization methods (Bendsøe \& Sigmund, 2003). 
The Topology Optimization Method (TOM) is a powerful tool to determine the better distribution of material within a fix design domain. Introduced by Bendsøe \& Kikuchi (1988), it combines optimization algorithms, e.g., Method of Moving Asymptotes (MMA) with Finite Element Analysis. The domain is discretized by finite elements, and considering the sensitivities regarding the proposed objective function and its restrictions, it is possible to obtain the optimal material arrangement. Figure 1.2 illustrates the complete optimization project for a diesel-generating set common base frame, where the topology optimization plays an important role on its weight reduction. From the design domain, it is defined which part of the component is fixed and which one is free to be optimized. Then, the component is modeled by finite elements and, applying a specific software, the topology optimization is conducted leading to the preliminary result. Next, the topology is interpreted regarding its final application. At this stage, it is usual to apply shape optimization to modify the boundaries of the structure, important, for instance, to reduce stress concentration. In shape optimization, the nodal coordinates are modified while the stresses in the component are constrained by stresses limits, e.g., yield stress. The optimized structure is validated when the stresses are below these limits for a certain tolerance. Otherwise, the project is reviewed until specific criteria are met.

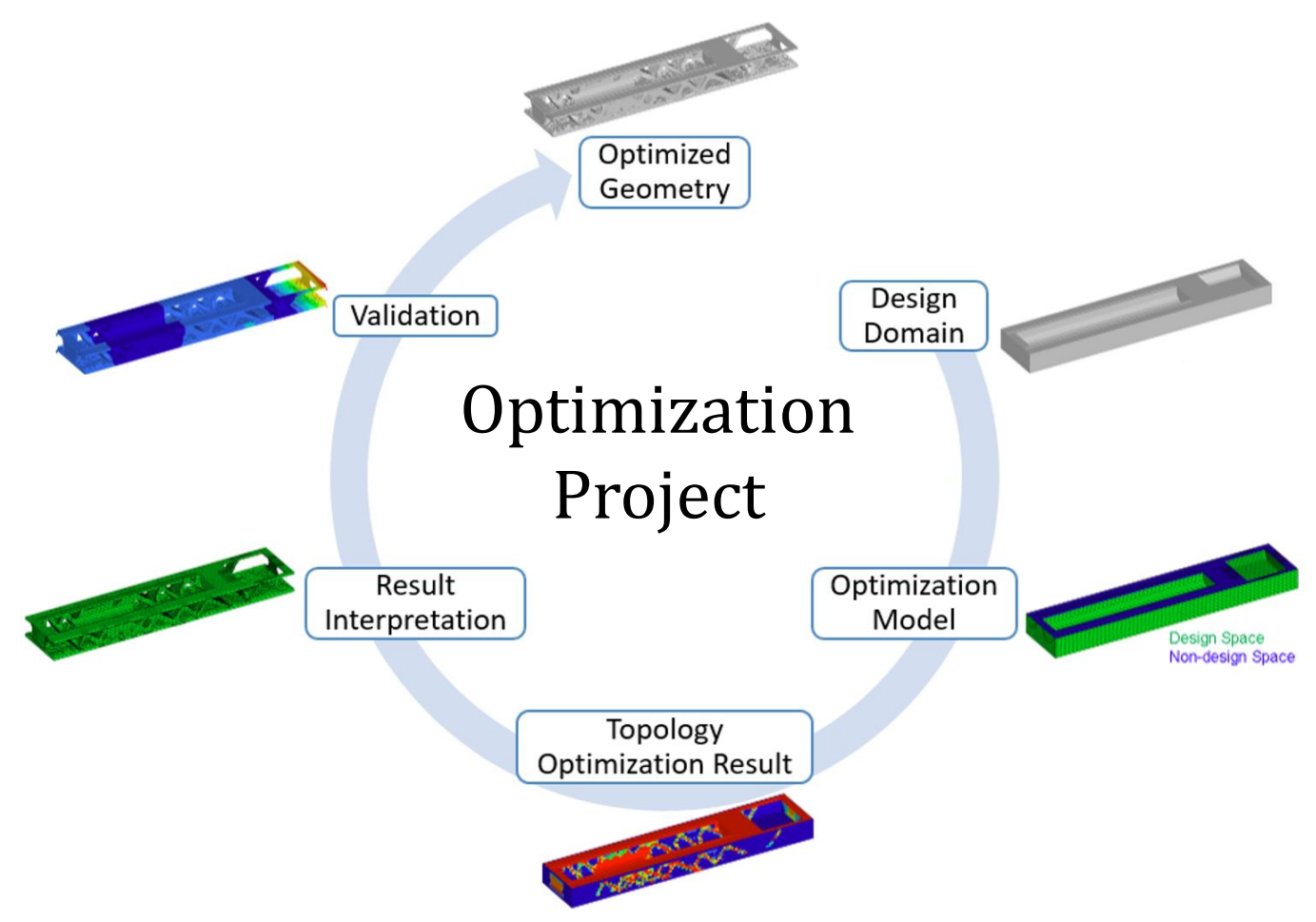

Figure 1.2: Illustrative scheme for a topology optimization project (adapted from VTT subproject (SIMPRO)). 
When the finite element analysis considers large displacements, the tangent stiffness matrix may become indefinite or even negative definite loss of ellipticity on the material law (see Ball, 1976). This phenomenon happens in low-density (also called low-stiffness or void) elements due to large displacements of their nodal coordinates and is known to be "artificial" since these elements should not influence the structural response (solid elements). Nonetheless, such finite displacements impose difficulties or even result in nonconvergence of the equilibrium iterations in the Newton-Raphson solution or similar scheme.

A easier way to comprehend this phenomenon is observing that large displacements can distort and flip void elements into themselves, resulting in negative volume. This is not evaluated by the classical Continuum Mechanics theory, in which the strain measures, based on the deformation gradient, has only physical meaning when a body is not turned inside out. Therefore, this thesis studies a technique to reduce the distortions of low-density regions and allows the nonlinear structural topology algorithm to converge.

\section{1}

\section{Motivation}

Nonlinear topology design has several applications in the design of components and equipments with large displacements or large deformations. The aeronautical industry due to the intrinsic need to design light structures is an area in which components are commonly projected using topology optimization. An example is the design of a rib structure in the fixed leading edge of an airplane, as showed in Figure 1.3.

Another example is the project of structures based on energy absorption. After a collision, a vehicle is subjected to a wide range of energy, resulting in large deformations in its bodywork. While designing a car, concerning, say, its weight reduction, the TOM considering the nonlinear theory can be employed and this method must take into account the energy absorbed by the vehicle in case of impact and be capable to protect its occupants (crash-worthiness). Other examples can be cited as flexible prostheses in Figure 1.4, MicroElectro-Mechanical Systems (MEMS), compliant mechanisms, high-pressure tanks subject to buckling and many other industrial applications (see Lahuerta, 2012). 

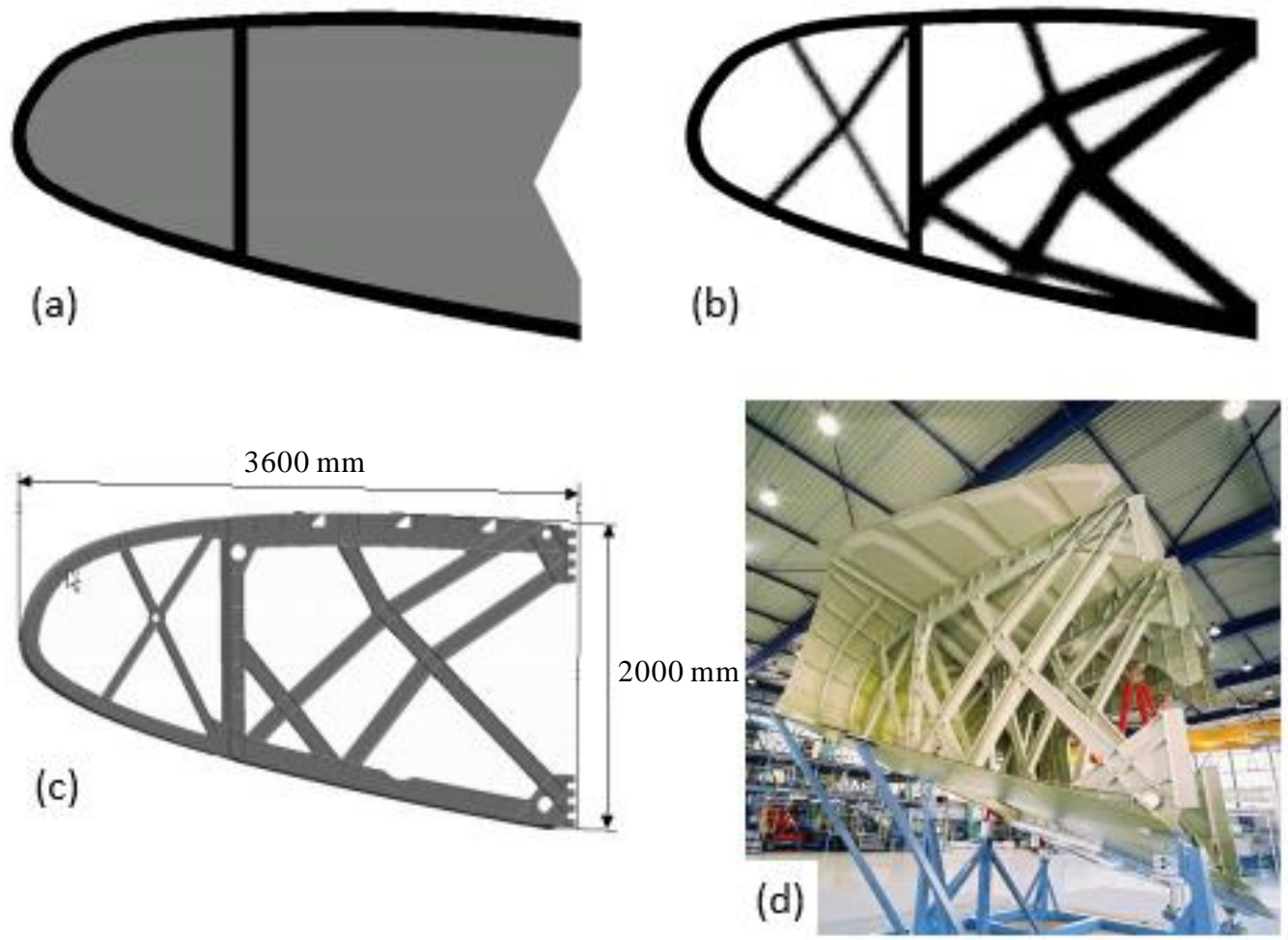

Figure 1.3: Optimization process of a rib structure in the front part of the wing of Airbus A380: (a) design domain; (b) optimized layout; (c) actual structure; (d) manufactured wing structure (Bendsøe \& Sigmund, 2007).

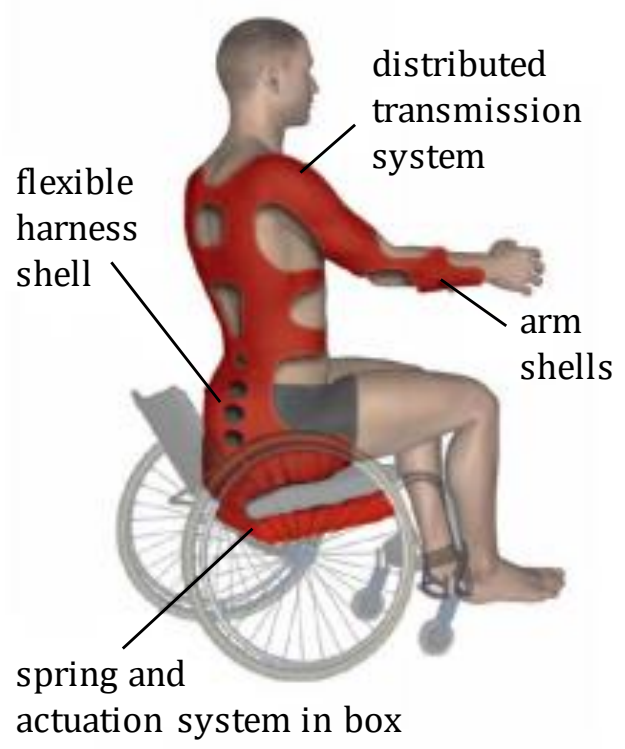

Figure 1.4: Body support structure to help a disabled person lift his arm (adapted from Holtzer (2017)).

Nonlinear topology optimization is a very challenging area. The biggest problem is dealing with difficulties in the convergence of the nonlinear solution 
algorithm. Nonetheless there are also complications in the implementation of the nonlinear finite element method consistently, since this requires either a different formulation of material law, capable of correctly describing the behavior in excessive compression, or the study of specific techniques to bypass large distortions in low-stiffness regions, as considered in this thesis. These complications, in turn, do not occur in analyses considering infinitesimal displacements and circumvent them is essential to achieve coherent responses in designing structures with nonlinear behavior.

\section{2 \\ Objective}

According to the examples mentioned in Section 1.1, nonlinear topology design is a field of wide and varied applications. To alleviate numerical instabilities in the finite element simulations of nonlinear topology optimization, and ensure its convergence, this thesis studies the technique proposed by Wang et al. (2014).

The discussed method can be applied to the traditional St. VenantKirchhoff (SVK) material law without the solution algorithm resulting in non-convergence of the optimization routine. To ensure that the equilibrium path is properly traced, and the structure reaches the maximum load, the incremental-iterative Newton-Raphson Procedure, NRP, is employed. The mesh is discretized by 4-node quadrilateral, Q4, elements. The optimization algorithm should be robust, such as OC (Bendsøe \& Sigmund, 2003) or MMA (Svanberg, 1987), and the continuation method and a nonlinear projection technique are employed to reduce the gray scale (intermediate densities) in the final structure.

\section{3}

\section{Literature Review}

Most of the works in literature are focused on linear elastic behavior, meaning that the structure has small displacements and also small deformations. This assumption is applicable to a variety of problems. However, a linear assumption is not always coherent, and those are the cases when studying space antennas or compliant mechanisms (Bruns \& Tortorelli, 2001). Topology optimization is a useful tool in the conceptual design phase, which allows the designer to obtain the optimum result at an early stage of the design process. For these reasons, there is a growing need to develop a powerful method for topology optimization, including nonlinearities. In the last two decades, several essays have appeared in the area of nonlinear optimization considering structures 
submitted to large displacements and/or large deformations to solve, mainly, the "end-compliance" minimization problem. Some of these are: Jog (1996), Bruns \& Tortorelli (1998, 2001, 2003), Buhl et al. (2000), Kemmler et al. (2005), Lahuerta et al. (2013), Wang et al. (2014), Luo et al. (2015) and Wallin et al. (2018). Dealing with both geometric and material nonlinearities, to name a few, there are the works by Jung \& Gea (2004), Huang \& Xie (2008), Lee \& Park (2012) and Luo \& Tong (2016).

The first study to consider nonlinear regime in topology optimization was that of Jog (1996). His article was centered on presenting the formulation for large displacements considering mechanical and thermal loads, although the author did not show significant differences in the results from linear and nonlinear topologies. Bruns \& Tortorelli (1998) and Bruns \& Tortorelli (2001) formulated a hyperelastic material law. In the first, a modified St. VenantKirchhoff (mSVK) material law was proposed to make the elements stiffer for large compressive deformations in void areas. In the latter, the SVK model was used, but no strategy to deal with the convergence problem was demonstrated. As consequence, all the solutions are limited to certain load level because the deformation cannot be too extreme.

Buhl et al. (2000) cited explicitly the difficulty to solve the nonlinear equilibrium equation in topology optimization. Considering that convergence is guaranteed when nodal displacement is below a defined tolerance and proved that numerical instabilities happen in nodes surrounded by void elements, the authors suggested to remove these degrees of freedom on the convergence criterion defined for the Newton-Raphson procedure. This idea though may reduce the solution accuracy and even yields in incorrect topologies.

Removing void elements from the finite element mesh reduces the size of the problem since the design domain is reduced. However this strategy has serious drawbacks: (a) the sensitivity analysis cannot be conducted in void elements; (b) in the course of the optimization, elements may reappear in the mesh and be disconnected from the others; (c) re-meshing may be necessary due to the change in the design domain. Bruns \& Tortorelli (2003) proposed a technique to remove and reintroduce elements at each optimization iteration, which proved to be very efficient in reducing convergence difficulties, despite of this strategy seems to have high costs. Recently, Luo \& Tong (2016) came up with a new strategy without the need of re-meshing and avoiding disconnected structures. The discussed algorithm was capable of solving the nonlinear compliance minimization problem. The applied technique is very promising, nevertheless needs further investigation.

During the optimization process, local unstable structures could appear 
in the design domain (e.g., local or global buckling). The Newton-Raphson method may fail in determining this equilibrium points when the tangent stiffness matrix in close to zero or negative. If such is the case, a possibility is to analyze whether the structural stiffness decreases or not in course of the optimization analysis. This approach was investigated by Kemmler et al. (2005). The stiffness matrix was verified on each load increment to determine if the optimum structure was stable. In unstable points, the Newton-Raphson procedure was replaced by the arc-length method.

The work of Lahuerta et al. (2013) used the neo-Hookean (nH) material law proposed by Simo-Ciarlet in structures with geometric nonlinearities. The St. Venant-Kirchhoff material law is known to provide a physically inconsistent response to large compressions resulting in convergence problems in areas of low-density materials (see Lahuerta, 2012). For meshes discretized by Q4 elements, which are more sensitive to mesh distortion (see Long et al., 2009), the nH models presented different results compared to the SVK-based formulation in high load levels. Even though making use of hyperelastic models, numerical instabilities were not completely solved, and the difficulties associated to large displacements optimization problem were treated invoking a relaxation function.

Wang et al. (2014) suggested an energy interpolation scheme. The idea was to interpolate the strain energy density between the large deformation formulation and the small deformation formulation. Assuming, ideally, void regions have very small density, these areas can be modeled in any desired way so they will not influence the solid elements. Hence, the structural part was discretized according to the nonlinear assumption and the low-stiffness regions were modeled based on the linear deformation premises. Based on this idea, Wallin et al. (2018) investigated what happened when the secant stiffness matrix was applied to the nonlinear solution scheme and compared the results to the tangent stiffness matrix. In both cases, numerical instabilities in lowstiffness regions were reduced, even at high load levels.

Pajot (2006) discussed another interpolation approach where the element residual force vector is directly interpolated, by a homotopic parameter, between the geometrically linear and nonlinear elastic residuals. One of the difficulties regarded to this method is the choice of the homotopic parameter, which varies from problem to problem. Wang et al. (2014) had investigated this approach and commented that it was still susceptible to numerical instabilities, such as the negative determinant of the deformation gradient $J$.

Another interpolation was formulated by Luo et al. (2015), in which a model of hyperelastic material is added to the design domain. This idea has 
the great advantage of being easy to incorporate into commercial softwares, as recently done by Chen et al. (2018). Therefore, two distinct meshes were superimposed: one discretized by the elastic material of St. Venant-Kirchoff (other models can be considered) and another - the additive mesh - defined by the hyperelastic material, in which the Yeoh model was selected. To make this technique coherent, the design variables (densities) of the additive mesh must assume small values. In the literature of reference, some researchers, for example Lahuerta et al. (2013), commented that the simple use of a hyperelastic material model - other than the SVK - did not completely solve the numerical instabilities in void regions. Yet, by controlling the maximal von Mises strain, Luo et al. (2015) were able to control the parameter $c_{2}$ in Yeoh model at each iteration of the optimization process. The authors reported reduction of numerical instabilities and that such interpolation technique was effective to alleviate excessive distortions in low-density and intermediatedensity elements. An in deeper look at this technique is needed, as it has not been proven that the approximation of the remodeled structure is sufficiently accurate.

A few papers discussed the use of optimization algorithms other than MMA or OC: Gea \& Luo (2001) and Jung \& Gea (2004) studied the minimization of mean compliance using the generalized convex approximation algorithm for a micro-structure-based design domain; Gomes \& Senne (2014) formulated a new algorithm named Sequential Piecewise Linear Programming (SPLP) on an intention of combine the SLP method, that has cheap iterations but many steps to achieve convergence, and the SQP method, that takes smaller number of iterations although is slower than the SLP method.

Besides those previously mentioned studies, other articles investigated the problem for different biases. Huang \& Xie (2008) proposed an improved Bi-directional Evolutionary Optimization (BESO) method to maximize the structural stiffness under prescribed displacements. Kawamoto (2009) replaced the Newton-Raphson procedure by the Levenberg-Marquardt method. Yoon \& Kim (2005a) formulated a technique where all the finite elements within the design domain are kept solid: Element Connectivity Parameterization (ECP). In this method, the elements are connected to each other through zero-length elastic links. These links are the design variable to be penalized on the optimization procedure. The optimized structures are comparable to those in the literature (e.g., Buhl et al., 2000; Gomes \& Senne, 2014). Yoon et al. (2011) employed the ECP formulation to prescribed load-displacement curves in different structures, including an example of snap-through phenomenon. On the essay of van Dijk et al. (2014) another interpolation method called 
Element Deformation Scaling, EDS, was proposed. By defining new examples and did not show the value of the objective function, most of the results are incomparable with other references. Despite these methods having fewer iterations in Newton-Raphson and optimization procedures when compared to the SIMP method (Section 3.2.1), both ECP and EDS are still susceptible to convergence problems in nonlinear topology optimization cases.

\section{4}

\section{Outline}

The organization of this thesis is as follows:

Chapter 2 forges some concepts of Continuum Mechanics, as well as a brief review on the finite element method, dedicated to geometric nonlinear problems;

Chapter 3 discuss and develop the main features of a density-based topology optimization approach including SIMP, continuation scheme, gray scale, checkerboard pattern and mesh-dependency and non-uniqueness of solution. Regularization techniques are also presented in this chapter;

Chapter 4 formulates the topology optimization under geometric nonlinearity for the "end-compliance" problem. Here the interpolation scheme to deal with convergence difficulties is discussed. The sensitivity analysis and the nonlinear methods of load control, arc-length and generalized displacement control are also formulated in this chapter;

Chapter 5 shows the results for traditional examples used in nonlinear topology optimization analyses: cantilever and clamped beams;

Chapter 6 express the final considerations, as well as proposes issues to be investigated in future works. 


\section{2 \\ A Brief Review on the Finite Element Method}

\section{1}

\section{Introduction}

Finite Element Method (FEM) is widely used and diffused in engineering analyses. Its basis was fostered in solving problems of structural mechanics. Later it was recognized that this technique could be applied in the solution of other classes of problems. Finite element procedures can be employed to study problems of solids and structures, heat transfer and fluids, mainly when the case being analyzed has no analytical solution or when this solution is complicated to be determined (which is the case of a multi-degree-of-freedom problem).

The formulation presented here is the standard theory, based on displacements, which is more effective to deal with most of practical problems, except those discretized by plate or shell elements and incompressible media. For these classes of problems, the mixed interpolation approach is more acceptable (Bathe, 2014). Only the isoparametric formulation will be discussed in this chapter.

In the first part of this chapter, a brief review on Continuum Mechanics is presented. Then, the geometric nonlinear finite element theory will be discussed, as well as the isoparametric formulation for plane elements and their corresponding interpolation functions. At last, the Gauss numerical integration is introduced.

\section{2}

\section{Mechanics of Deformable Bodies}

A body in three-dimensional Euclidean space, like the one indicated in Figure 2.1, is composed by a set of particles. For a given geometry and loading, this body will undergo macroscopic deformations. If the applied load are timedependent, then the deformation will be a function of time as well. If the loads are applied slowly, so the deformation is only load-dependent.

At time $t=0$, the body has the configuration ${ }^{0} \Omega$ and it is composed by the particles $P$ and $Q$, occupying positions described, respectively, by the 
vectors $\boldsymbol{X}$ and $\boldsymbol{X}+d \boldsymbol{X}$. The vector $\boldsymbol{X}$ has coordinates $\left(X_{1}, X_{2}, X_{3}\right)$, called material coordinates. The material coordinate system, indicated in Figure 2.1, will be used in the examples throughout this thesis unless stated otherwise.

After the deformation, at a time $t$, this body has the configuration ${ }^{t} \Omega$, in which the particles $p$ and $q$ are defined by $\boldsymbol{x}$ and $\boldsymbol{x}+d \boldsymbol{x}$, and $\boldsymbol{x}$ has coordinates $\left(x_{1}, x_{2}, x_{3}\right)$, known as spatial coordinates. The relation describing the motion is

$$
\boldsymbol{x}=\boldsymbol{X}+\boldsymbol{u},
$$

where $\boldsymbol{u}$ denotes the displacement.

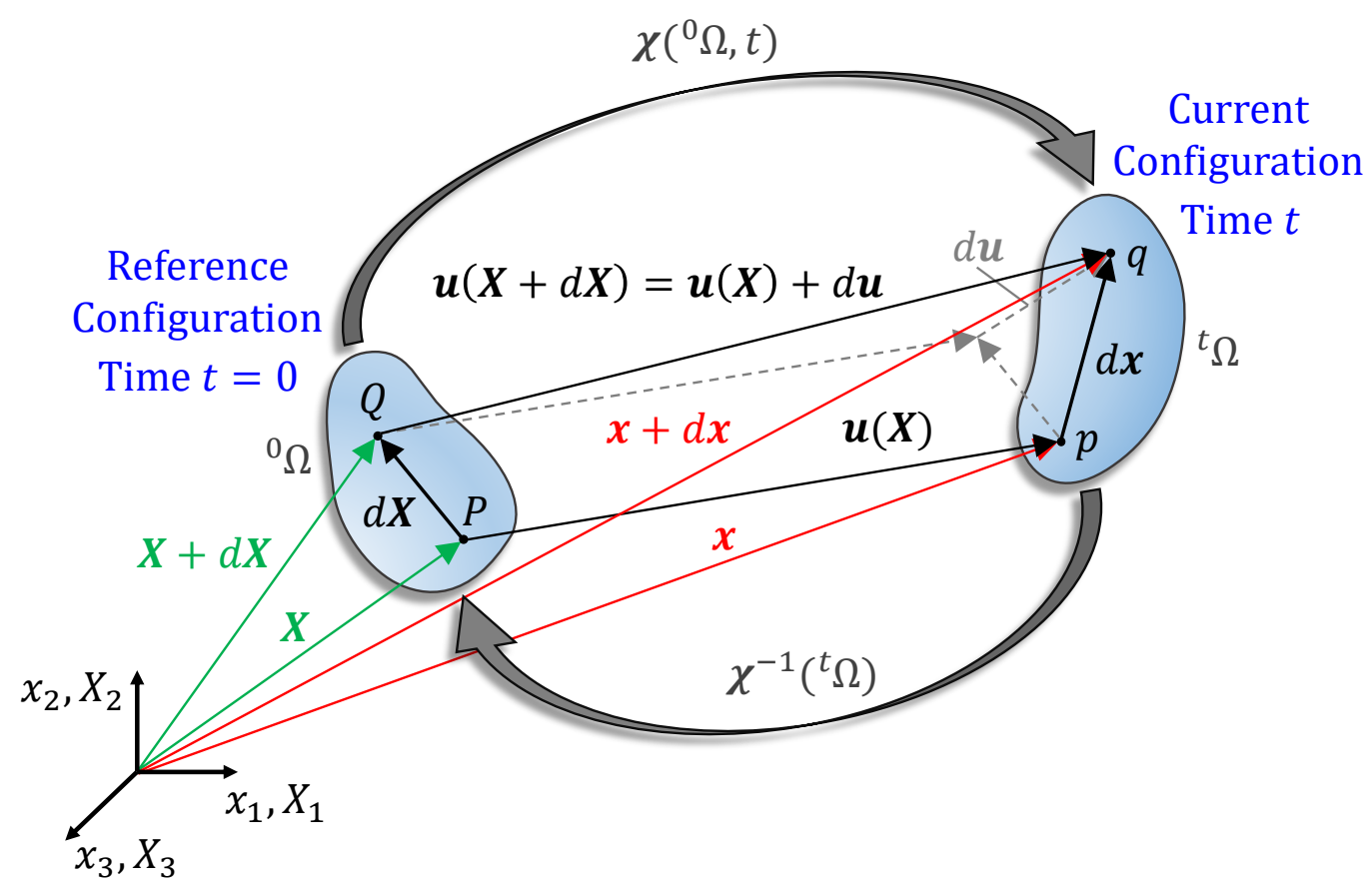

Figure 2.1: Schematic motion and deformation of a solid in space.

When the motion is described from the reference or initial configuration to the current or deformed configuration, the classical Continuum Mechanics define the motion as the Lagrangian or material description. However, the relation in Equation (2.1) is reversible and one can observe this motion looking back for the initial position: from the current or deformed configuration to the reference one. That is the Eulerian or spatial description. The Eulerian description appears in fluid mechanics because it is more adequated to study a fixed region in space, i.e., a control volume. In solid mechanics, as the initial position is known, the Lagrangian description is the natural choice, and, since this thesis is about structural optimization, this is the description addressed in following discussion. 
The deformation mapping $\boldsymbol{\chi}$ maps the body from the reference to current configuration, i.e., the deformation mapping $\chi\left({ }^{0} \Omega, t\right)$ takes every position vector $\boldsymbol{X}$ belonging to the initial configuration ${ }^{0} \Omega$ and places these points in the deformed configuration as $\boldsymbol{x}=\boldsymbol{\chi}\left({ }^{0} \Omega\right)$. The deformation gradient $\boldsymbol{F}$ gives the relation of a material line $d \boldsymbol{X}$ to a spatial line $d \boldsymbol{x}$ (consisting of the same material as $d \boldsymbol{X}$ )

$$
d \boldsymbol{x}=\boldsymbol{F} d \boldsymbol{X}
$$

where

$$
\boldsymbol{F}=\nabla_{0} \boldsymbol{x}=\frac{\partial \boldsymbol{x}}{\partial \boldsymbol{X}}
$$

or, in index notation,

$$
F_{i J}=\frac{\partial x_{i}}{\partial X_{J}}=x_{i, J}
$$

The determinant of $\boldsymbol{F}$ is $J=\operatorname{det}(\boldsymbol{F})$, which is important to quantify the volume ratio of current to initial configuration. The gradient deformation is a 2 nd-order tensor, thus is possible to write its inverse, $\boldsymbol{F}^{-\mathbf{1}}$, to describe the inverse transformation $d \boldsymbol{X}=\boldsymbol{F}^{-1} d \boldsymbol{x}$ :

$$
F_{J i}^{-1}=\frac{\partial X_{J}}{\partial x_{i}}=X_{J, i}
$$

Those interested in a more in-depth reading on Continuum Mechanics can consult, among several traditional readings, Mase \& Mase (1999) and Reddy (2008).

\subsection{1}

\section{Strain Measures}

The geometric changes in a continuous medium can be quantified in a variety of ways. Here, the most traditional strain measures will be discussed.

Look again to the particles $P$ and $Q$, separated by $d \boldsymbol{X}$, in the reference configuration and to the particles $p$ and $q$, separated by $d \boldsymbol{x}$, in the current configuration. To measure the deformation induced by certain motion, it is often to use the quadratic distance between two particles. In each configuration, this distance is defined as 


$$
\begin{gathered}
(d S)^{2}=d \boldsymbol{X} \cdot d \boldsymbol{X} \\
(d s)^{2}=d \boldsymbol{x} \cdot d \boldsymbol{x}=d \boldsymbol{X} \cdot\left(\boldsymbol{F}^{T} \boldsymbol{F}\right) d \boldsymbol{X}
\end{gathered}
$$

where the term in parenthesis is called right Cauchy-Green deformation tensor,

$$
\boldsymbol{C}=\boldsymbol{F}^{T} \boldsymbol{F} \quad \text { or } \quad C_{I J}=F_{k I} F_{k J}
$$

which is a symmetric 2nd-order tensor. Analogously, the left Cauchy-Green deformation or Finger tensor, $\boldsymbol{b}$, is

$$
\boldsymbol{b}=\boldsymbol{F} \boldsymbol{F}^{T} \quad \text { or } \quad b_{i j}=F_{i K} F_{j K}
$$

One common strain measure is based on the difference in squared lengths. For the reference configuration, this relation can be easily derived using index notation and Kronecker delta:

$$
\begin{aligned}
\|d \boldsymbol{x}\|^{2}-\|d \boldsymbol{X}\|^{2} & =\left(F_{k I} d X_{I}\right)\left(F_{k J} d X_{J}\right)-d X_{I} d X_{J} \\
& =F_{k I} F_{k J} d X_{I} d X_{J}-\delta_{I J} d X_{I} d X_{J} \\
& =\left(C_{I J}-\delta_{I J}\right) d X_{I} d X_{J} .
\end{aligned}
$$

The term in parenthesis is

$$
2 \boldsymbol{E}=\boldsymbol{C}-\boldsymbol{I} \quad \text { or } \quad 2 E_{I J}=C_{I J}-\delta_{I J}
$$

where $\boldsymbol{E}$ is the Green-Lagrangian strain tensor and $\boldsymbol{I}$ is the identity matrix. The idea described in Equation (2.10) can be also derived for spatial variables:

$$
\begin{aligned}
\|d \boldsymbol{x}\|^{2}-\|d \boldsymbol{X}\|^{2} & =d x_{i} d x_{j}-\left(F_{K i}^{-1} d x_{i}\right)\left(F_{K j}^{-1} d x_{j}\right) \\
& =\delta_{i j} d x_{i} d x_{j}-F_{K i}^{-1} F_{K j}^{-1} d x_{i} d x_{j} \\
& =\left(\delta_{i j}-b_{i j}^{-1}\right) d x_{i} d x_{j},
\end{aligned}
$$

The expression in the parenthesis is 


$$
2 \boldsymbol{e}=\boldsymbol{I}-\boldsymbol{b}^{-1} \quad \text { or } \quad 2 e_{i j}=\delta_{i j}-b_{i j}^{-1}
$$

in which, $\boldsymbol{e}$ the Almansi-Eulerian strain tensor.

Both Green and Almansi tensors are independent from rigid body motion. They can be written in terms of displacements by differentiating the Equation (2.1) and applying the definition of $\boldsymbol{C}$ and $\boldsymbol{b}$ in Equations (2.11) and (2.13). This will result in the expressions,

$$
\begin{aligned}
& \boldsymbol{E}=\frac{1}{2}\left[\nabla_{0} \boldsymbol{u}+\left(\nabla_{0} \boldsymbol{u}\right)^{T}+\left(\nabla_{0} \boldsymbol{u}\right)^{T} \nabla_{0} \boldsymbol{u}\right] \text { or } \\
& E_{I J}=\frac{1}{2}\left(u_{I, J}+u_{J, I}+u_{k, I} u_{k, J}\right) \\
& \boldsymbol{e}=\frac{1}{2}\left[\nabla \boldsymbol{u}+(\nabla \boldsymbol{u})^{T}-(\nabla \boldsymbol{u})^{T} \nabla \boldsymbol{u}\right] \text { or } e_{i j}=\frac{1}{2}\left(u_{i, j}+u_{j, i}-u_{K, i} u_{K, j}\right),
\end{aligned}
$$

where the subscript $\nabla_{0}$ denotes that the differentiation is with respect to the material coordinates $\boldsymbol{X}$.

In Equations (2.14) and (2.15) is very easy to identify the portion neglected by infinitesimal displacement theory, i.e., the high-order terms. Thus, the infinitesimal strain measure is

$$
\boldsymbol{\varepsilon}=\frac{1}{2}\left[\nabla_{0} \boldsymbol{u}+\nabla_{0} \boldsymbol{u}^{T}\right] \quad \text { or } \quad \varepsilon_{i j}=\frac{1}{2}\left(u_{i, j}+u_{j, i}\right)
$$

The rate of deformation of Green-Lagrangian strain tensor is

$$
\dot{\boldsymbol{E}}=\frac{1}{2}\left(\dot{\boldsymbol{F}}^{T} \boldsymbol{F}+\boldsymbol{F}^{\boldsymbol{T}} \dot{\boldsymbol{F}}\right)
$$

\subsection{2}

\section{Stress Measures}

After addressing the strain tensors, it is important to introduce the stress measures. The Cauchy stress $\boldsymbol{\sigma}$ is a 2 nd-order symmetric tensor, so $\sigma_{i j}=\sigma_{j i}$ is true. It relates a force in the current configuration to an area in the deformed state. A more formal definition for the Cauchy tensor, relating it to the traction vector, can be found in Mase \& Mase (1999). Other stress measures are introduced in the study of large deformations. The first and the second Piola-Kirchhoff stress tensors are: 


$$
\begin{array}{ccc}
\boldsymbol{P}=J \boldsymbol{\sigma} \boldsymbol{F}^{-T} & \text { or } & P_{i I}=J \sigma_{i j} F_{I j}^{-1} \\
\boldsymbol{S}=\boldsymbol{F}^{-1} \boldsymbol{P} & \text { or } & S_{I J}=F_{I k}^{-1} P_{k J} .
\end{array}
$$

The first Piola-Kirchhoff stress tensor $\boldsymbol{P}$ relates forces in the current configuration with the corresponding areas in the reference configuration. So, it can be interpreted as the current force per unit of undeformed area. It belongs neither to the reference nor to the current configuration and, thus, it is consider a mixed tensor. In general, this tensor is unsymmetric. In turn, the second Piola-Kirchhoff stress tensor $\boldsymbol{S}$ is contrived to produce a totally material symmetric tensor. This stress measure relates forces to areas in the reference configuration.

Sometimes it can be inconvenient to calculate the stresses as a function of $\operatorname{det}(J)$, since it also depends on the deformation. So, another stress measure, that has a similar transformation relation with Equation (2.19), can be introduced:

$$
\boldsymbol{\tau}=J \boldsymbol{\sigma}=\boldsymbol{F} \boldsymbol{S} \boldsymbol{F}^{T} \quad \text { or } \quad \tau_{i j}=J \sigma_{i j}=F_{i K} S_{K L} F_{j L},
$$

for $\boldsymbol{\tau}$ being the Kirchhoff stress. It is worth to mention that under small deformation theory all these four stress measures are equivalent.

\section{3}

\section{Finite Element Formulation in Nonlinear Problems}

There are, essentially, three sources of nonlinearities in solid mechanics: geometric, from material and kinematic. In kinematic (or boundary) nonlinearity the boundary conditions change during the motion of the body under consideration. One example refers to two bodies in contact, in which the displacements on the contact boundary are limited so they cannot penetrate each other. Therefore, this source is also referred to as "contact problems". In materiallynonlinear-only case, the nonlinearity come from the fact that the stress-strain relation is not linear. Geometric nonlinearity, studied in this work, arises from the fact that the relations displacement/strains and rotation/strains are nonlinear.

The basic problem in nonlinear analysis is to find the equilibrium state of a body corresponding to the external force vector $\boldsymbol{l}$,

$$
l-f=0,
$$


in which $\boldsymbol{f}$ is the vector of internal force vector. This relation express the equilibrium of the system accounting all nonlinearities.

When the analysis depends on the time or the material conditions and properties or the (nonlinear) path, as in this thesis, the condition in Equation (2.21) must be satisfied for the complete history of loads (or prescribed displacements). Then, an incremental-iterative procedure, discussed in Section 4.5, is applied in order to guarantee the equilibrium at every step of the solution until the maximum load (or displacement) is reached.

In the analysis of nonlinear finite elements, a common difficulty is how to describe the equilibrium of a body in the current configuration, since it is previously unknown. Thus, it is necessary to establish the relation between the equilibrium of the body in the current and in the reference configuration. This is possible by using an incremental formulation and the principle of virtual work. This principle states that if a body is in equilibrium, for any small deformation, the internal virtual work is equal to the external virtual work, that is,

$$
\int_{t_{\Omega}} \boldsymbol{\sigma}: \overline{\boldsymbol{E}} \mathrm{d} v=\int_{t_{\Omega}} \boldsymbol{f}^{B} \cdot \overline{\boldsymbol{u}} \mathrm{d} v+\int_{t_{\Gamma}} \boldsymbol{t} \cdot \overline{\boldsymbol{u}} \mathrm{d} a,
$$

where $\overline{\boldsymbol{u}}$ is the virtual displacement vector, $\overline{\boldsymbol{E}}$ is the variation of Lagrangian strain and the double dot symbol ":" is the contraction operator. $\boldsymbol{f}^{B}$ is the applied force per unit deformed volume ${ }^{t} \Omega$ and $\boldsymbol{t}$ is the vector traction (force per unit deformed area) that acts on an element on the surface ${ }^{t} \Gamma$. The terms $\mathrm{d} v$ and $\mathrm{d} a$ represent, respectively, an infinitesimal element of volume and area in the current configuration.

Substituting Equation (2.18) into (2.19) and using the relation (2.17), after few manipulations (Bonet \& Wood, 2008), the Equation (2.22) can be rewritten as

$$
\int_{t_{\Omega}} \boldsymbol{\sigma}: \overline{\boldsymbol{E}} \mathrm{d} V=\int_{0_{\Omega}} \boldsymbol{S}: \overline{\boldsymbol{E}} \mathrm{d} V=\int_{{ }_{0} \Omega} \boldsymbol{f}_{0}^{B} \cdot \overline{\boldsymbol{u}} \mathrm{d} V+\int_{{ }^{0}} \boldsymbol{t}_{0} \cdot \overline{\boldsymbol{u}} \mathrm{d} A
$$

on what $\boldsymbol{f}_{0}^{B}=J \boldsymbol{f}^{B}$ is the body force per unit initial volume ${ }^{0} \Omega$ and $\boldsymbol{t}_{0}$ is the traction vector defined in material coordinates and acting on a region ${ }^{0} \Gamma . \mathrm{d} V$ and $\mathrm{d} A$ correspond, respectively, to the infinitesimal element of volume and area in the reference configuration.

In Equation (2.23) it was assumed that the applied forces are conservative, therefore the applied loads are independent of the deformation. It follows from the work conjugate pair $\{\boldsymbol{S}, \dot{\boldsymbol{E}}\}$ (Bonet \& Wood, 2008) and respects the 
principle of objectivity which, roughly speaking, establishes that the law of material must be invariant to the rigid body motion since it does not induce deformations. Hence, internal forces should not be affected by superposed rigid body motions.

\subsection{1}

\section{Linearization of Incremental Equations}

It is essential to write the incremental finite element equation. To develop a governing linearized equation, it is important to emphasize that, for a body in the current configuration, all the previous states are known and could be applied in the linearization. Nevertheless, in practice, two formulations have been used: the Total Lagrangian (TL) and the Updated Lagrangian (UL). In the last, the solution scheme for all static and kinematic variables are referred to the last configuration calculated. The TL formulation refers to parameters in the initial configuration and it is also named as Lagrangian formulation. Here, only the TL formulation will be discriminated. The loading is also assumed to be deformation-independent, i.e., all applied loads are concentrated, which means the directions and the intensities are constant and independent of the structural response.

To linearize the incremental equations, the concept of directional derivative, $D\left(W_{\text {int }}(\overline{\boldsymbol{u}})\right)[\delta \boldsymbol{u}](\mathrm{Kim}, 2015)$, is applied to the left-hand side of Equation (2.23):

$$
\begin{aligned}
D\left(W_{\text {int }}(\overline{\boldsymbol{u}})\right)[\delta \boldsymbol{u}] & =\int_{0_{\Omega}} D(\overline{\boldsymbol{E}}: \boldsymbol{S})[\delta \boldsymbol{u}] \mathrm{d} V \\
& =\int_{0_{\Omega}} \overline{\boldsymbol{E}}: D(\boldsymbol{S})[\delta \boldsymbol{u}] \mathrm{d} V+\int_{0_{\Omega}} \boldsymbol{S}: D(\boldsymbol{E})[\delta \boldsymbol{u}] \mathrm{d} V \\
& =\int_{0_{\Omega}} \overline{\boldsymbol{E}}: D\left(\left.\frac{\partial \boldsymbol{S}}{\partial \boldsymbol{E}} \frac{\partial \boldsymbol{E}}{\partial \varrho}\right|_{\varrho=0}\right)[\delta \boldsymbol{u}] \mathrm{d} V+\int_{0_{\Omega}} \boldsymbol{S}: D(\overline{\boldsymbol{E}})[\delta \boldsymbol{u}] \mathrm{d} V \\
& =\int_{0_{\Omega}} \overline{\boldsymbol{E}}: \mathcal{C}^{0}: D(\overline{\boldsymbol{E}})[\delta \boldsymbol{u}] \mathrm{d} V+\int_{0_{\Omega}} \boldsymbol{S}: D(\overline{\boldsymbol{E}})[\delta \boldsymbol{u}] \mathrm{d} V . \quad
\end{aligned}
$$

The term $[\delta \boldsymbol{u}]$ represents the direction of the linearization and $\varrho$ its the corresponded magnitude. The elasticity tensor $\mathcal{C}^{0}$ is given by $\partial \boldsymbol{S} / \partial \boldsymbol{E}$ and $\partial \boldsymbol{E} / \partial \varrho$ follows immediately from the definition of directional derivative:

$$
D(\boldsymbol{E}(\overline{\boldsymbol{u}}))[\delta \boldsymbol{u}]=\left.\frac{\partial}{\partial \varrho} \boldsymbol{E}(\overline{\boldsymbol{u}}+\varrho \delta \boldsymbol{u})\right|_{\varrho=0}
$$

The term $\overline{\boldsymbol{E}}$ can be composed into the sum of linear and nonlinear 
portions, respectively,

$$
\begin{gathered}
\overline{\boldsymbol{E}}_{L}=\frac{1}{2}\left(\nabla_{0} \delta \boldsymbol{u}+\nabla_{0} \delta \boldsymbol{u}^{T}+\nabla_{0} \overline{\boldsymbol{u}}^{T} \nabla_{0} \delta \boldsymbol{u}+\nabla_{0} \delta \boldsymbol{u}^{T} \nabla_{0} \overline{\boldsymbol{u}}\right) \\
\overline{\boldsymbol{E}}_{N L}=\frac{1}{2}\left(\nabla_{0} \delta \boldsymbol{u}^{T} \nabla_{0} \delta \boldsymbol{u}\right) .
\end{gathered}
$$

The first term in Equation (2.24) are function of $\overline{\boldsymbol{u}}$ and $\delta \boldsymbol{u}$. This term is linear with respect to $\delta \boldsymbol{u}$ but nonlinear with respect to the virtual displacement $\overline{\boldsymbol{u}}$. Nonlinearity comes from the fact that the stress and strain implicitly depend on $\overline{\boldsymbol{u}}$. Thereby, by neglecting the nonlinear term, $\overline{\boldsymbol{E}}_{N L}$, in the first term of Equation (2.24) and recalling the external forces are deformation-independent, the linearized principle of virtual work is:

$$
\int_{{ }_{0} \Omega} \overline{\boldsymbol{E}}: \mathcal{C}^{0}: D\left(\overline{\boldsymbol{E}}_{L}\right)[\delta \boldsymbol{u}] \mathrm{d} V+\int_{0} \boldsymbol{S}: D\left(\overline{\boldsymbol{E}}_{N L}\right)[\delta \boldsymbol{u}] \mathrm{d} V=\boldsymbol{l}-\boldsymbol{f}
$$

The right-hand side of Equation (2.28) is the residual or unbalanced force vector $\boldsymbol{r}$, in which

$$
\begin{gathered}
\boldsymbol{l}=\int_{{ }_{0} \Omega} \boldsymbol{f}_{0}^{B} \cdot \overline{\boldsymbol{u}} \mathrm{d} V+\int_{{ }^{0} \Gamma} \boldsymbol{t}_{0} \cdot \overline{\boldsymbol{u}} \mathrm{d} A \\
\boldsymbol{f}=\int_{{ }_{0} \Omega} \boldsymbol{S}: D\left(\overline{\boldsymbol{E}}_{L}\right)[\delta \boldsymbol{u}] \mathrm{d} V,
\end{gathered}
$$

or simply,

$$
\boldsymbol{K}[\delta \boldsymbol{u}]=\boldsymbol{r}
$$

where $\boldsymbol{K}$ is the tangent stiffness matrix. Equation (2.31) has to be solved according to an incremental-iterative method. For further detail among the linearization, one can read Bathe (2014) or Kim (2015).

\subsection{2}

\section{Element Isoparametric Formulation}

The idea is to relate the geometry described in two different coordinate systems: one local and other global. This can be done using Lagrangian interpolation or shape functions. The isoparametric formulation has this name because both nodal coordinates and element displacements are interpolated by the same shape functions. 


\subsubsection{1}

\section{Quadrilateral Elements}

The representation of geometry can be considered as a mapping procedure that transforms a square shape in local coordinate system to a regular quadrilateral or distorted shape in global coordinate system, as shown in Figure 2.2 for a twodimensional case. In the local coordinate system are used natural coordinates $\left(\xi_{1}, \xi_{2}\right)$, each one of them varying from -1 to 1 . The global coordinate system is commonly the Cartesian coordinate system, for example $\left(X_{1}, X_{2}\right)$ in the reference configuration.

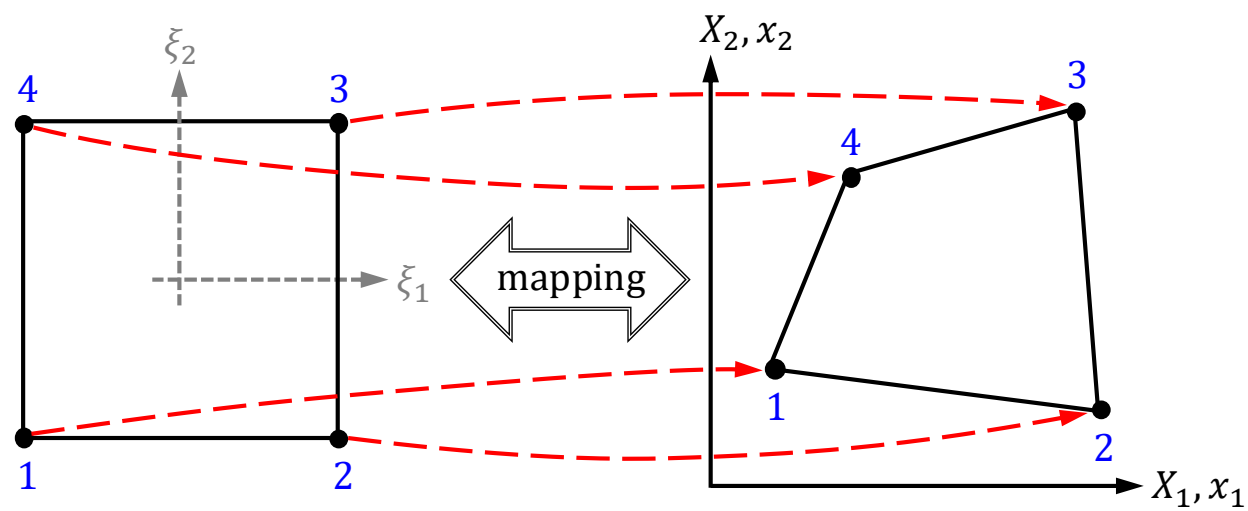

Figure 2.2: Mapping of a square in local coordinate system to an arbitrary straight-sided quadrilateral in global coordinate system.

The element discretization in the reference configuration is interpolated considering the initial geometry described in terms of the points $X_{j}^{i}$. The superscript $i$ represents the nodal points of a certain element and the subscript $j$ represents the degree of freedom. For now on, the two-dimensional case will be considered. So, the element interpolated coordinates $X_{j}$ are

$$
X_{j}=\sum_{i=1}^{n} h_{i}\left(\xi_{1}, \xi_{2}\right) X_{j}^{i}, \quad \text { for } j=1,2
$$

where $h_{i}$ is the interpolation function written in natural coordinates. A similar relation can be denoted for elements in the current configuration. Thus:

$$
x_{j}=\sum_{i=1}^{n} h_{i}\left(\xi_{1}, \xi_{2}\right) x_{j}^{i}, \quad \text { for } j=1,2
$$

The interpolation function $h_{i}$ has a fundamental property: in the natural coordinate system, $h_{i}$ values one at node $i$ and zero in the others. Plus, the summation of all shape functions of an element must be unitary. Considering 
the general 2D element in Figure 2.3, the corresponding interpolation functions are given in Table 2.1 .

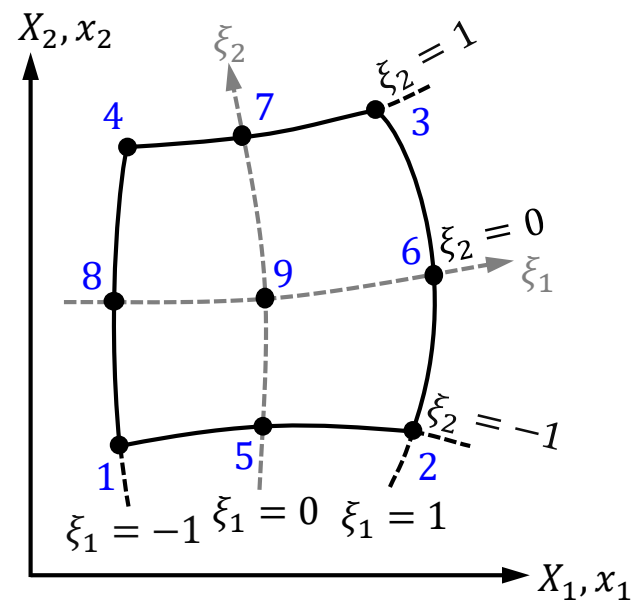

Figure 2.3: Representation of a 4-to-9-node quadrilateral element.

Table 2.1: Interpolation functions for quadrilateral elements (adapted from Bathe (2014)).

Included only if node $i$ is defined

\begin{tabular}{l|c|c|c|c|c|} 
& $i=5$ & $i=6$ & $i=7$ & $i=8$ & $i=9$ \\
\hline$h_{1}=\frac{1}{4}\left(1-\xi_{1}\right)\left(1-\xi_{2}\right)$ & $-\frac{1}{2} h_{5}$ & & & $-\frac{1}{2} h_{8}$ & $-\frac{1}{4} h_{9}$ \\
$h_{2}=\frac{1}{4}\left(1+\xi_{1}\right)\left(1-\xi_{2}\right)$ & $-\frac{1}{2} h_{5}$ & $-\frac{1}{2} h_{6}$ & & & $-\frac{1}{4} h_{9}$ \\
$h_{3}=\frac{1}{4}\left(1+\xi_{1}\right)\left(1+\xi_{2}\right)$ & & $-\frac{1}{2} h_{6}$ & $-\frac{1}{2} h_{7}$ & & $-\frac{1}{4} h_{9}$ \\
$h_{4}=\frac{1}{4}\left(1-\xi_{1}\right)\left(1+\xi_{2}\right)$ & & & $-\frac{1}{2} h_{7}$ & $-\frac{1}{2} h_{8}$ & $-\frac{1}{4} h_{9}$ \\
\hline$h_{5}=\frac{1}{2}\left(1-\xi_{1}^{2}\right)\left(1-\xi_{2}\right)$ & & & $-\frac{1}{2} h_{9}$ \\
\hline$h_{6}=\frac{1}{2}\left(1-\xi_{2}^{2}\right)\left(1+\xi_{1}\right)$ & & & $-\frac{1}{2} h_{9}$ \\
\hline$h_{7}=\frac{1}{2}\left(1-\xi_{1}^{2}\right)\left(1+\xi_{2}\right)$ & & & $-\frac{1}{2} h_{9}$ \\
\hline$h_{8}=\frac{1}{2}\left(1-\xi_{2}^{2}\right)\left(1-\xi_{1}\right)$ & & & \\
\hline$h_{9}=\left(1-\xi_{1}^{2}\right)\left(1-\xi_{2}^{2}\right)$ & & \\
\hline
\end{tabular}

The element displacements are interpolated in the same way as the nodal coordinates. Therefore,

$$
u_{j}=\sum_{i=1}^{n} h_{i}\left(\xi_{1}, \xi_{2}\right) u_{j}^{i}, \quad \text { for } j=1,2
$$

in which $u_{j}^{i}$ is the displacement at node $n$ for the $j$ degrees of freedom. 
In order to calculate the stiffness matrix, it is necessary to determine the strain-displacement relation. It is easy to observe from Equations (2.33) and (2.34) that both coordinates and displacements are functions of type $g=f\left(\xi_{1}, \xi_{2}\right)$, for $g$ representing coordinates $X_{1}$ and $X_{2}$ or displacements $u_{1}$ and $u_{2}$. The chain rule is the appropriate choice to describe the transformation of coordinates. For $X_{j}$-axis, this transformation is of the form

$$
\frac{\partial}{\partial X_{j}}=\frac{\partial}{\partial \xi_{1}} \frac{\partial \xi_{1}}{\partial X_{j}}+\frac{\partial}{\partial \xi_{2}} \frac{\partial \xi_{2}}{\partial X_{j}}
$$

where the terms $\partial \xi_{k} / \partial X_{j}$, for $j, k=1,2$, are unknowns. Observing the chain rule above, to solve the inverse derivatives the following matrix form can be written:

$$
\left[\begin{array}{c}
\frac{\partial}{\partial \xi_{1}} \\
\frac{\partial}{\partial \xi_{2}}
\end{array}\right]=\left[\begin{array}{ll}
\frac{\partial X_{1}}{\partial \xi_{1}} & \frac{\partial X_{2}}{\partial \xi_{1}} \\
\frac{\partial X_{1}}{\partial \xi_{2}} & \frac{\partial X_{2}}{\partial \xi_{2}}
\end{array}\right]\left[\begin{array}{c}
\frac{\partial}{\partial X_{1}} \\
\frac{\partial}{\partial X_{2}}
\end{array}\right]
$$

or most directly,

$$
\frac{\partial}{\partial \boldsymbol{\xi}}=\boldsymbol{J}_{0} \frac{\partial}{\partial \boldsymbol{X}}
$$

where $\boldsymbol{J}_{0}$ is Jacobian matrix, responsible for relating natural coordinate derivatives to the global ones. If the inverse of $\boldsymbol{J}_{0}$ exists, the following operation can be applied to determine the terms in the last column of Equation (2.36):

$$
\frac{\partial}{\partial \boldsymbol{X}}=\boldsymbol{J}_{0}^{-1} \frac{\partial}{\partial \boldsymbol{\xi}}
$$

Proved the non-singularity of the Jacobian matrix, an important characteristic is revealed. The map relating natural coordinates $\left(\xi_{1}, \xi_{2}\right)$ to Cartesian coordinates, in both reference $\left(X_{1}, X_{2}\right)$ and current $\left(x_{1}, x_{2}\right)$ configurations, is unique. In other words, a certain point described in natural coordinates has only one representation in the global coordinate system and vice versa.

When large displacements are allowed to happen in a finite element analysis, the elements can become highly distorted and even flip over themselves. In such case, the Jacobian matrix $\boldsymbol{J}_{0}$ becomes singular and consequently the isoparametric formulation is no longer valid. 


\subsection{3}

\section{Matrix Form}

Equation (2.31), for static problems, can be written in matrix form. The tangent stiffness matrix is represented in left-hand-side of Equation (2.28). The first integrand can be written as

$$
\boldsymbol{K}^{E}=\bigcap_{e=1}^{N_{e}} \int_{\Omega_{e}}\left(\boldsymbol{B}_{L 0}+\boldsymbol{B}_{L 1}\right)^{T} \boldsymbol{D}^{0}\left(\boldsymbol{B}_{L 0}+\boldsymbol{B}_{L 1}\right) \mathrm{d} V,
$$

where $\bigcap$ is the assembly operator acting from the eth finite element to $N_{e}$ (number of elements in the mesh), $\Omega_{e}$ is the element domain, $\boldsymbol{D}^{0}$ is the matrix representation of the Elasticity tensor $\mathcal{C}^{0}$.

Equation (2.39) can be further decomposed by splitting its terms. Then, the following expression arises:

$$
\begin{aligned}
\boldsymbol{K}^{E}= & \underbrace{\bigcap_{\boldsymbol{N}_{e}}^{N_{e}}\left[\int_{\Omega_{e}} \boldsymbol{B}_{L 0}^{T} \boldsymbol{D}^{0} \boldsymbol{B}_{L 0} \mathrm{~d} V\right]}_{\boldsymbol{K}^{L}}+ \\
& \underbrace{}_{\bigcap_{e=1}^{N_{e}}\left[\int_{\Omega_{e}}\left(\boldsymbol{B}_{L 0}^{T} \boldsymbol{D}^{0} \boldsymbol{B}_{L 1}+\boldsymbol{B}_{L 1}^{T} \boldsymbol{D}^{0} \boldsymbol{B}_{L 0}+\boldsymbol{B}_{L 1}^{T} \boldsymbol{D}^{0} \boldsymbol{B}_{L 1}\right) \mathrm{d} V\right]}
\end{aligned}
$$

where the $\boldsymbol{K}^{L}$ in the linear stiffness matrix (the same arising from infinitesimal displacement's problems) and $\boldsymbol{K}^{D}$ is the "initial displacement" stiffness matrix. For the $n$th nodal point, the strain-displacement matrices, depicted above, are

$$
\begin{gathered}
\boldsymbol{B}_{L 0_{n}}=\left[\begin{array}{cc}
h_{n, \xi_{1}} & 0 \\
0 & h_{n, \xi_{2}} \\
h_{n, \xi_{2}} & h_{n, \xi_{1}}
\end{array}\right], \\
\boldsymbol{B}_{L 1_{n}}=\left[\begin{array}{cc}
l_{11} h_{n, \xi_{1}} & l_{21} h_{n, \xi_{1}} \\
l_{12} h_{n, \xi_{2}} & l_{22} h_{n, \xi_{2}} \\
l_{11} h_{n, \xi_{2}}+l_{12} h_{n, \xi_{1}} & l_{21} h_{n, \xi_{2}}+l_{22} h_{n, \xi_{1}}
\end{array}\right]
\end{gathered}
$$

and

$$
l_{i j}=\sum_{k=1}^{n} h_{k, \xi_{j}} u_{i}^{k} \text {, for } i, j=1,2 .
$$


The elasticity tensor depends on which material model is considered. For hyperelastic materials, in which exists a strain energy density function $\phi$, it is defined as the second derivative of $\phi$ with respect to the strain measure, e.g., $\mathcal{C}_{I J K L}^{0}=\partial^{2} \phi / \partial E_{I J} \partial E_{K L}$. However, the SVK model presents a linear relationship between the stress and strain measures. The elasticity tensor is constant and can be written as function of any elastic modulus:

$$
\boldsymbol{S}=\mathcal{C}^{0}: \boldsymbol{E}
$$

The relation of Equation (2.44) can be rewritten in the matrix notation

$$
\hat{\boldsymbol{S}}=\boldsymbol{D}^{0} \hat{\boldsymbol{E}}
$$

where $\hat{\boldsymbol{S}}$ and $\hat{\boldsymbol{E}}$ are, respectively, the vector representation of $\boldsymbol{S}$ and $\boldsymbol{E}$ according to the Voigt notation. For a plane strain state and employing the Young's modulus $E_{0}$ and Poisson's coefficient $\nu_{0}$ the matrix form of $\mathcal{C}^{0}$ is

$$
\boldsymbol{D}^{0}=\frac{E_{0}\left(1-\nu_{0}\right)}{\left(1+\nu_{0}\right)\left(1-2 \nu_{0}\right)}\left[\begin{array}{ccc}
1 & \frac{\nu_{0}}{1-\nu_{0}} & 0 \\
\frac{\nu_{0}}{1-\nu_{0}} & 1 & 0 \\
0 & 0 & \frac{1-2 \nu_{0}}{2\left(1-\nu_{0}\right)}
\end{array}\right]
$$

Returning to Equation (2.28), the second integrand, on the other hand, appears in geometric nonlinear problems. It is defined as:

$$
\boldsymbol{K}^{S}=\bigcap_{e=1}^{N_{e}} \int_{\Omega_{e}} \boldsymbol{B}_{N L}^{T} \tilde{\boldsymbol{S}} \boldsymbol{B}_{N L} \mathrm{~d} V .
$$

Since it depends on the stress measure, $\boldsymbol{K}^{S}$ is called the initial stress stiffness.

$$
\boldsymbol{B}_{N L_{n}}=\left[\begin{array}{cc}
h_{n, \xi_{1}} & 0 \\
h_{n, \xi_{2}} & 0 \\
0 & h_{n, \xi_{1}} \\
0 & h_{n, \xi_{2}}
\end{array}\right]
$$

and 


$$
\tilde{\boldsymbol{S}}=\left[\begin{array}{cccc}
S_{11} & S_{12} & 0 & 0 \\
S_{21} & S_{22} & 0 & 0 \\
0 & 0 & S_{11} & S_{12} \\
0 & 0 & S_{21} & S_{22}
\end{array}\right]
$$

Finally, by combining the terms defined in Equations (2.40) (2.47), the tangent stiffness matrix is

$$
\boldsymbol{K}=\boldsymbol{K}^{L}+\boldsymbol{K}^{D}+\boldsymbol{K}^{S}
$$

The right-hand side of Equation (2.28), representing the internal forces, is given by:

$$
\boldsymbol{f}=\bigcap_{e=1}^{N_{e}} \int_{\Omega_{e}}\left(\boldsymbol{B}_{L 0}+\boldsymbol{B}_{L 1}\right)^{T} \hat{\boldsymbol{S}} \mathrm{d} V .
$$

\section{4}

\section{Numerical Integration}

The finite element formulation requires integration over the element domain $\Omega_{e}$ to compute, for example, the stiffness matrices and vector forces. Most integrals are difficult to be analytically evaluated, thus it is often faster to integrate them numerically. Among many numerical integration methods that have been proposed, the Gauss integration rule is commonly used in the finite element formulation due to its simplicity and accuracy.

Considerer a two-dimensional function $f(x, y)$ defined, by convenience, in the same interval of isoparametric finite elements: $[-1,1]$. This function is approximated by the Gauss integration as

$$
\int_{-1}^{1} \int_{-1}^{1} f(x, y) \mathrm{d} x \mathrm{~d} y \approx \sum_{i=1}^{N G} \sum_{j=1}^{N G} \omega_{i} \omega_{j} f\left(x_{i}, y_{j}\right)
$$

where $N G$ is the number of integration points, $\omega_{i}$ and $\left(x_{i}, y_{i}\right)$ are, respectively, the weight and the coordinate of the $i$ th sampling point.

The main task in Gauss quadrature (applied in isoparametric elements) is to choose the correct order of integration, i.e., to define the value of $N G$. Suppose the function $f(x)=x^{3}+7 x^{2}-5$ integrated in the limits $[a, b]$. Its primitive is 


$$
\int_{a}^{b} f(x) \mathrm{d} x=\left[\frac{1}{4} x^{4}+\frac{7}{3} x^{3}-5 x\right]_{b}^{a} .
$$

The minimum polynomial degree to exactly evaluate this integral ("full" integration) is 4 . So, the order of integration is given by the integral's primitive.

A common association can help defining the correct approximation: for NG sampling points a polynomial of degree $(2 N G-1)$ can be integrated exactly. However, this relation is not valid for triangular and tetrahedral elements, because the integration limits depends on the variables themselves.

Figure 2.4 illustrates the Gauss integration points in a Q4 regular element. Tables 2.2 displays the integration points and the weights for this type of element. And the integrals of Section 2.3.3 are rewritten in function of the natural coordinates $\left(\xi_{1}, \xi_{2}\right)$ :

$$
\int \mathrm{d} V=\int_{-1}^{1} \int_{-1}^{1} t \operatorname{det}\left(\boldsymbol{J}_{0}\right) \mathrm{d} \xi_{1} \mathrm{~d} \xi_{2}
$$

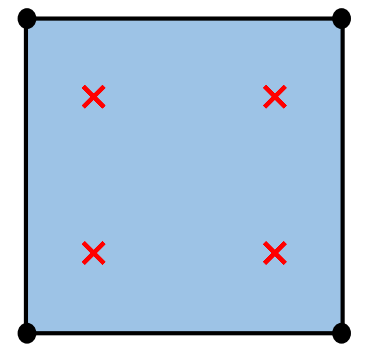

Figure 2.4: Gauss integration points of Q4 element.

Table 2.2: Gauss integration over Q4 element (adapted from Bathe (2014)).

\begin{tabular}{c|c|c|c}
$\begin{array}{c}\text { Integration } \\
\text { Order }(N G)\end{array}$ & $\begin{array}{c}\text { Degree of } \\
\text { Precision }\end{array}$ & Integration Points $\left(\xi_{1}, \xi_{2}\right)$ & Weights $(\omega)$ \\
\hline 1 & 1 & 0.0 & 2.0 \\
\hline 2 & 3 & \pm 0.5773502692 & 1.0 \\
\hline 3 & 5 & \pm 0.7745966692 & 0.5555555556 \\
\hline 4 & 7 & \pm 0.8611363116 & 0.3478546451 \\
& & \pm 0.3399810436 & 0.6521451549
\end{tabular}




\section{3}

\section{Topology Optimization}

\section{1}

\section{Introduction}

The concept of topology optimization was first introduced in the work of Bendsøe \& Kikuchi (1988), as an extension of shape optimization, which is limited to optimize only the boundaries of an initial topology. Shape optimization concerns control of nodal coordinates or the location of control points, as in the case where the boundaries are represented by curves (e.g., splines), to re-design the boundary of a structure.

Consider a certain design domain $\Omega$ as in Figure 3.1. Typically, one defines $\Omega$, where the boundary $\partial \Omega$ is divided into a portion $\Gamma_{D}$ over which the displacements are prescribed and the remainder is either free or subject to imposed traction $\boldsymbol{t}$. The amount of eliminated material in the domain is $\Omega_{v}=\Omega \backslash \Omega_{s}$, where the symbol " $\$ " is the relative complement, i.e., it represents the objects that belong to $\Omega$ and not to $\Omega_{s}$.

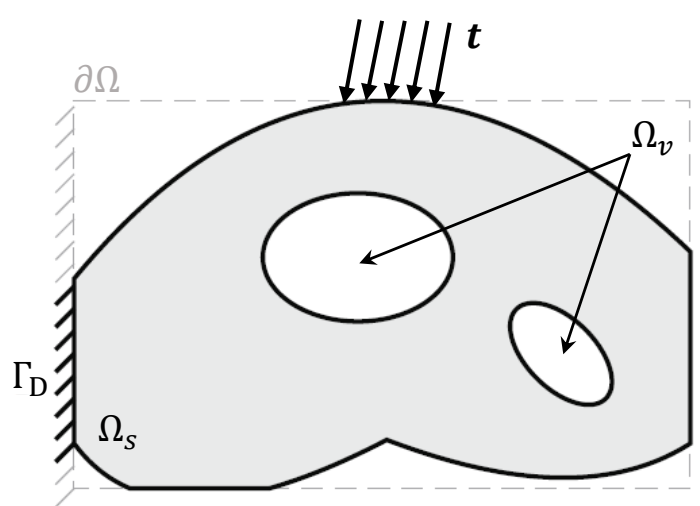

Figure 3.1: Representation of a topology optimization problem of design domain $\Omega$ (adapted from Talischi et al. (2012b)).

The topology optimization defines the optimum material distribution within a design domain with respect to prescribed loads and boundary conditions. The method consists in introducing and removing elements within $\Omega$ to reproduce, respectively, the removal and introduction of small holes, i.e., the material arrangement. The material distribution can be modeled by the 
homogenization method, which introduces composite micro-structure to the design space (Bendsøe \& Kikuchi, 1988), and the density approach (Bendsøe, 1989; Bendsøe \& Sigmund, 2003). In this work, the density-based technique is adopted, in which the geometry is parametrized by a material density function and the displacement field is approximated by finite elements.

In this chapter, the main aspects of this approach will be presented, as the material model based on SIMP and Continuation Scheme. It will be also discussed about regularization techniques used to solve some complications related to it, as gray scales and checkerboards in the final layout, and meshdependency and non-uniqueness of solution.

\section{2}

\section{Material Representation}

A typical approach, by computational means, and the one used in this thesis, is to discretize the design domain $\Omega$ using finite elements. Commonly, in the density approach, each element of the mesh assumes a value that represents the design variable, i.e., the "density", which is zero for voids and one for solid (or structural) elements.

Above, the word density is enclosed in quotation marks, because the design variable does not represent the density (ratio of mass to volume). However, over the years, it has been the most widely accepted interpretation in the literature Bendsøe \& Sigmund (2003).

A well-posed problem must satisfy, mainly, two conditions:

1. solution exists;

2. the solution is unique for the same initial data.

The discrete formulation (if no constraint is added, e.g., perimeter control) violates the condition 2 above. The $0-1$ problem is relaxed and the design variable is now assumed varying in the range $[0,1]$. This relaxation is also important to use gradient-based optimizers, such as MMA and OC.

\subsection{1}

\section{Solid Isotropic Material with Penalization (SIMP)}

The continuous parameterization of the design variables introduce intermediate values (gray regions) in the design domain. These values have no physical meaning, since the model represents an isotropic material (Bendsøe, 1989). The design variables must be penalized to recover the discrete characteristic. A widespread technique in topology optimization applications is the SIMP, Solid Isotropic Material with Penalization (Zhou \& Rozvany, 1991; Bendsøe \& Sigmund, 1999). Considering the density $\rho$, the SIMP method is 


$$
\tilde{\boldsymbol{x}}(\boldsymbol{z})=\epsilon+(1-\epsilon) \rho(\boldsymbol{z})^{p}, \quad \text { for } p \geqslant 1,
$$

in which $\epsilon$ is the Ersatz parameter. Typically, it assumes a small positive number, e.g., $10^{-4}$, to ensure non-singularity on the global stiffness matrix when $\rho(\boldsymbol{z}) \rightarrow 0$. The exponent $p$ is the penalization parameter and $\tilde{\boldsymbol{x}}(\boldsymbol{z})$ is called the material interpolation function.

The relation between unpenalized Elasticity tensor $\mathcal{C}_{I J K L}^{0}$ and the penalized one $\mathcal{C}_{I J K L}$ is written, in index notation, as

$$
\mathcal{C}_{I J K L}\left(z_{e}\right)=\tilde{x}_{e}\left(z_{e}\right) \mathcal{C}_{I J K L}^{0}
$$

for $\mathcal{C}_{I J K L}^{0}$ being characterized by the Young's modulus $E_{0}$ and the Poisson's coefficient $\nu$.

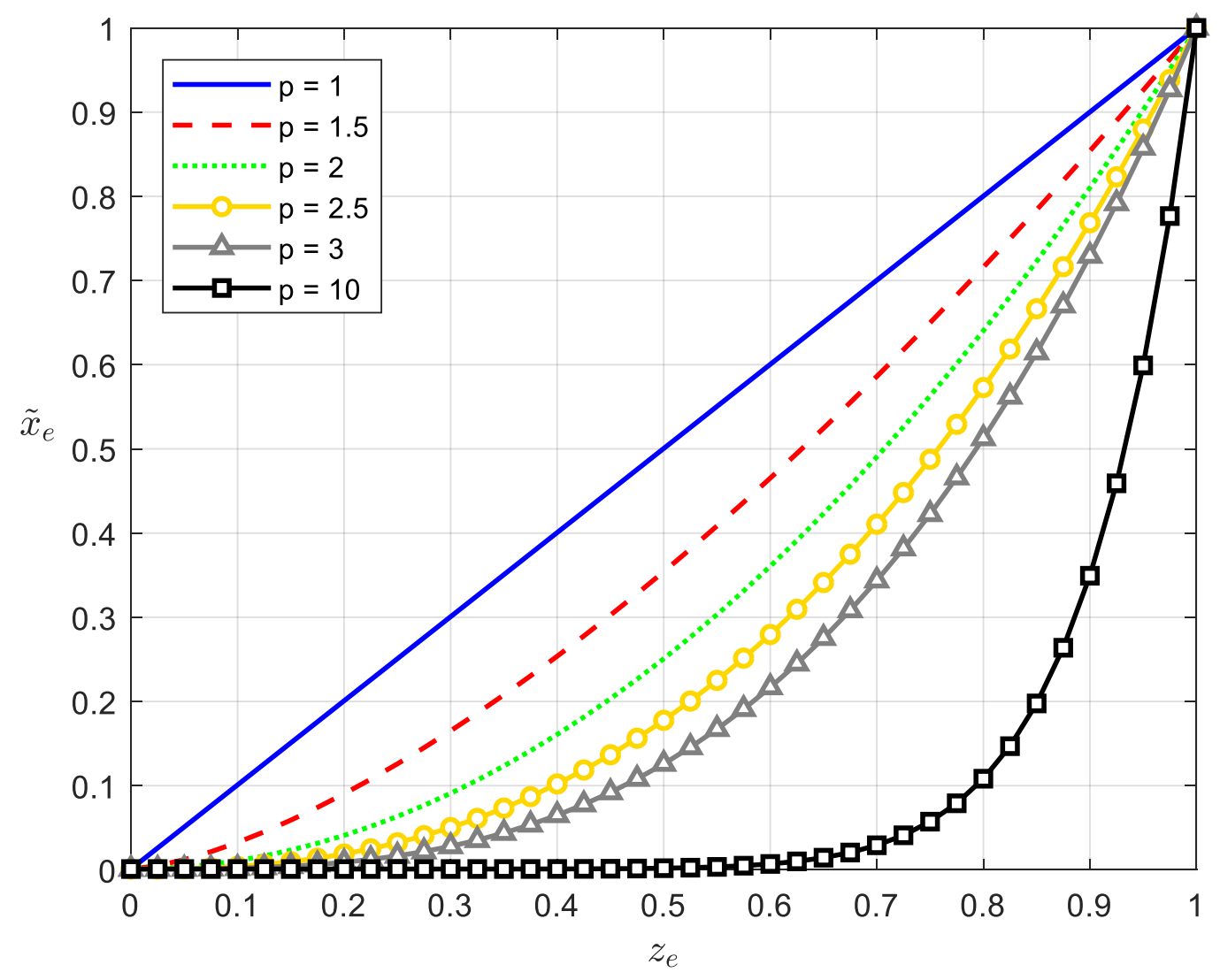

Figure 3.2: Influence of different penalization exponents in SIMP method.

In Figure 3.2 are illustrated different penalization exponents for an element $e$. As one can see, for $p=1$ there is no penalization in the eth design variable. As $p$ increases, the element design variable $z_{e}$ tends to recover its binary nature. In topology optimization, the maximum magnitude of $p$ is 
usually set in the range of $[3,5]$. Higher values approximates Equation (3.1) to the unitary step function $(p \rightarrow \infty)$, which steers the solution to discrete formulation and bring back all the difficulties associated to it.

\subsection{2}

\section{Continuation Scheme}

Most of topology optimization problems are non-convex, i.e., the solution is not guaranteed to converges to the global minimum. The idea of continuation is to gradually change an artificially convex (continuous) problem to the original non-convex (discrete), thus avoiding convergence to an undesirable local point (Sigmund \& Petersson, 1998).

In SIMP approach, the penalization parameter is, gradually, increased from $p=1$ to a certain limit, in this work $p=3$, by an increment $\Delta p$ :

$$
p_{i+1}=p_{i}+\Delta p
$$

Figure 3.3 illustrates how this method works. Function $f(\rho(\boldsymbol{z}))$ is convex, while $f\left(\rho(\boldsymbol{z})^{3}\right)$ has multiples local minima. Starting from a small value of $p$ increases the chances to obtain a final topology near to the global minimum.

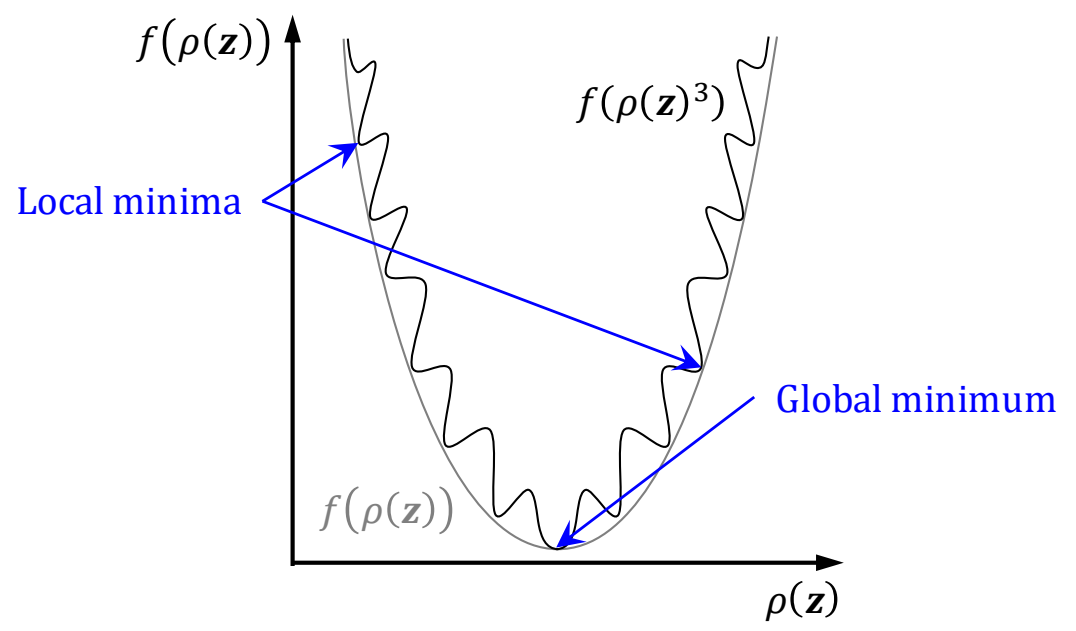

Figure 3.3: Schematization of continuation method (adapted from Lahuerta (2012)).

\section{3}

\section{Complications}

Three significantly important issues affect the computational results of topology design: gray scale, checkerboard layout and mesh-dependency. In this section these points will be discussed. 


\subsection{1}

\section{Gray Scale}

In the continuous formulation for topology optimization, the relaxation on the design variable introduce intermediate density values. These densities appear in the final layout as gray scales, cf. Figure 3.4. The grays tones are consistent with the applied formulation but, in most of the engineering problems, they have no applicability and should be eliminated from the final solution throughout techniques such as SIMP and projection methods.

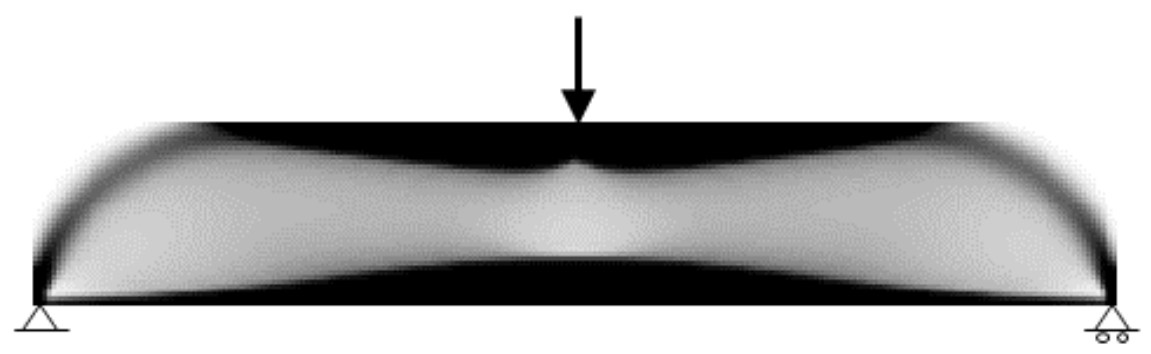

Figure 3.4: The MBB beam with gray scale. Result obtained without continuation or any penalization method.

\subsection{2}

\section{Checkerboard Pattern}

The pattern alternating solid and void material in checkerboard like fashion, cf. Figure 3.5, is known as checkerboard problem. The origin of this phenomenon is related to features of the finite element approximations that maximizes the strain energy (Diaz \& Sigmund, 1995). This layout has an overestimated artificially high stiffness which results in the optimum material arrangement in minimum compliance problems discretized by Q4-displacement elements.

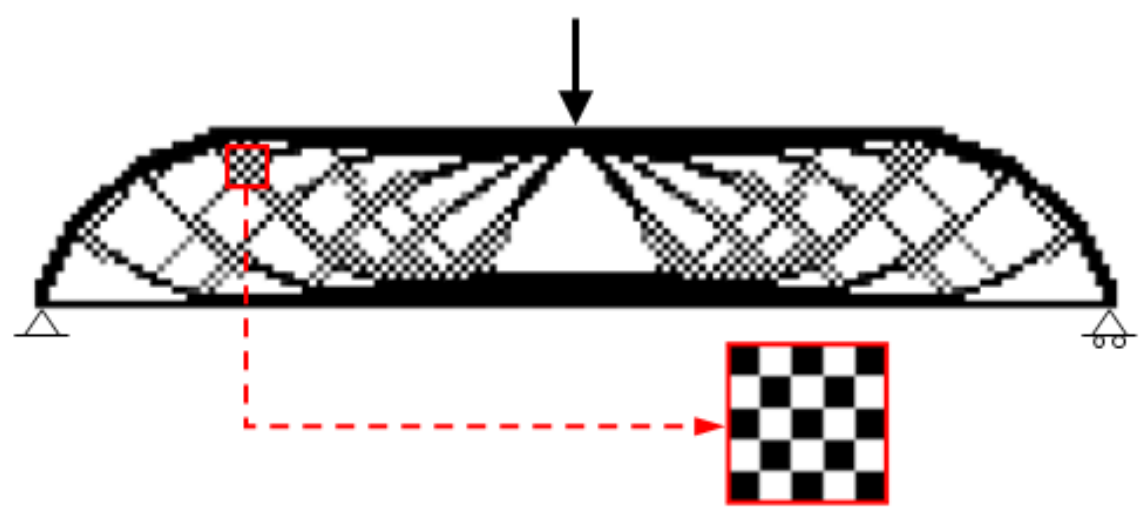

Figure 3.5: Checkerboard pattern demonstrated in the MBB beam.

The topology optimization is a two-field problem: densities $\rho(\boldsymbol{z})$ and displacements $\boldsymbol{u}$. Jog \& Haber (1996) reported that the checkerboard layout 
can be addressed to numerical instabilities caused by an inadequate choice of function spaces representing the fields of displacements and densities. This is similar to problems of fluid mechanics that violates the Ladyzenskaja-BabuskaBrezzi (LBB) condition (Talischi et al., 2014).

One way to avoid checkerboard-type pattern is to use higher-order elements. Four node elements are linearly interpolated; 8 or 9 node elements, for instance, are build by quadratic functions. Therefore, the application of higher-order elements increases the cost to evaluate CPU-time.

Polygonal elements have demonstrated to be very effective in dealing with checkerboards, as pointed by Talischi et al. (2009). Nevertheless, polygons contain functions of non-polynomial nature in their finite element spaces, which typically requires a large number of quadrature points to reduce consistency errors. For modeling nonlinear elasticity, the larger number of integration points required makes such approach less attractive from a practical point of view. On the other hand, the so-called Virtual Element Method (VEM) has been recently introduced in the literature (Chi et al., 2017). VEM is capable of handling general polygonal elements, including concave ones and, unlike FEM, the shape functions in VEM are constructed implicitly. In other words, for linear elements, only one integration point per element is required.

\subsection{3}

\section{Mesh-Dependency and Non-uniqueness of Solution}

Sigmund \& Petersson (1998) define: "Mesh-refinement should result in a better finite element modeling of the same optimal and a better description of boundaries - not in more detailed and qualitatively different structure".

The above statement is self-explanatory. When the refined mesh results in a different optimum structure the optimization procedure does not guarantee the uniqueness of solution.

Mesh-dependency is related to non-uniqueness of solution and meshrefinement. When refining the mesh, if mesh-independency is not guaranteed, the final topology will be dependable of the problem discretization, cf. Figure 3.6, where an improved finite element discretization results in a much more detailed structure. This means the final solution is not unique.

Some authors, as Bendsøe \& Sigmund (2003), refer to the fact of meshrefinement resulting in different responses as the non-existence of solutions. This is because by refining the mesh more and more, different optimum solutions are obtained, to the point where one no longer knows when the optimization procedure should stop. 


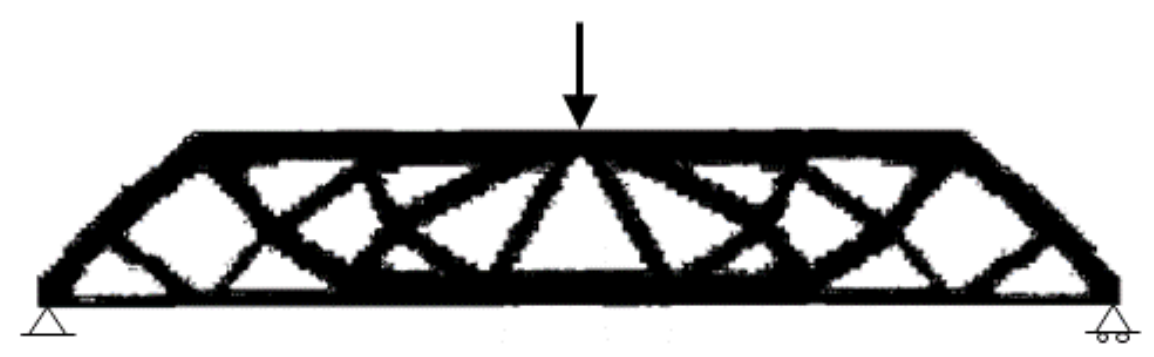

3.6(a): 2700 elements.

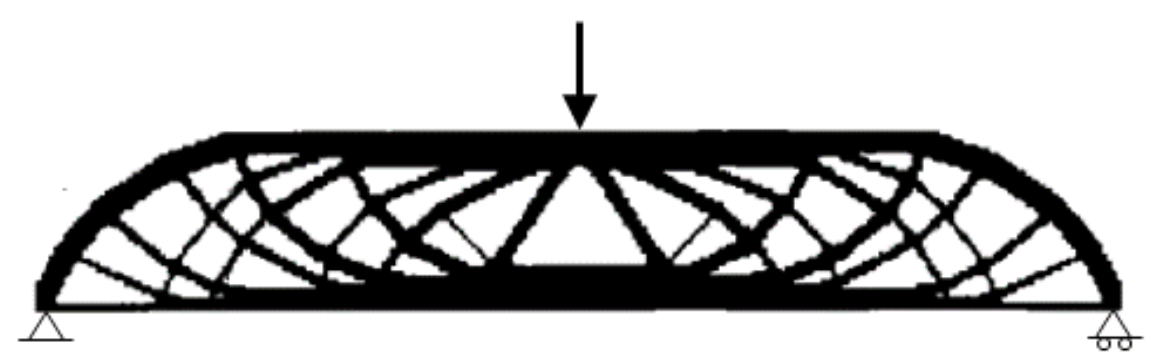

3.6(b): 4800 elements.

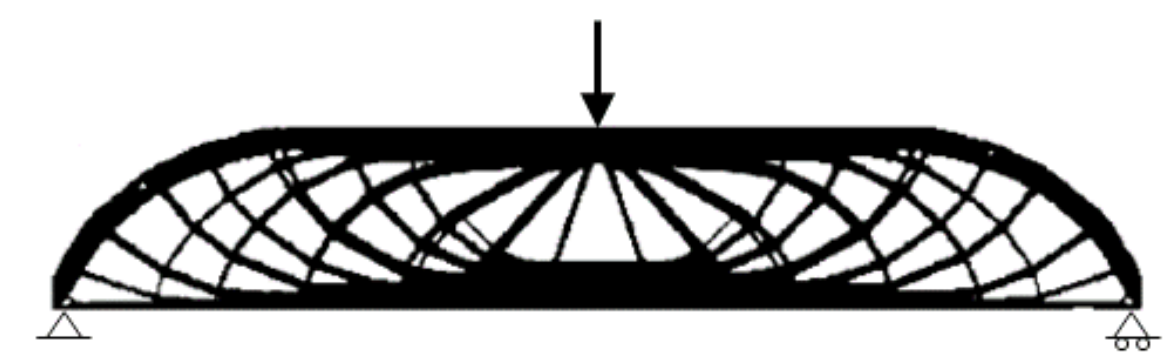

3.6(c): 17200 elements

Figure 3.6: Dependency of the optimal topology on mesh-refinement for the MBB beam for different mesh discretizations (adapted from Bendsøe \& Sigmund (2003)).

\section{4}

\section{Regularization Techniques}

In general, the introduction of more holes within the design domain, without changing the structural volume, will increase the efficiency of a given structure. Bendsøe \& Sigmund (2003) points out that in the limit of this process (of introducing more holes) one obtains structural variations in the form of microstructures that have an improved use of the material.

Regularization methods are employed to reduce the space of admissible designs by some sort of global or local restriction on the variation of design variable, thus difficulting the possibility of fine structures to formate. Those have been demonstrated very effective in yielding mesh-independent and checkerboard-free topologies. 


\subsection{1}

\section{Restriction Methods}

Restriction methods are those who impose a new constraint to the optimization problem (Bendsøe \& Sigmund, 2003). This is the case of perimeter control and gradient constraint, which eliminate checkerboards, at the same time the convergence to a final definitive solution is guaranteed. Nonetheless, for example, in problems where the volume is constrained, if such a restriction is not met, these methods become inadequate.

\subsubsection{1}

\section{Perimeter Control}

The perimeter control (Haber et al., 1996) imposes one constraint in the optimization problem. The total perimeter is restricted allowing to control the number of admissible holes within the design domain.

As this method enforces a global constraint, this is still susceptible to local problems, as the formation of very thin bars. Furthermore, the determination of the perimeter itself is empirical: a small value of it may not satisfy a certain constraint, e.g., the volume, and a high value will introduce more holes than the necessary. This makes difficult to apply this method to practical problems, where the final layout is unknown.

\subsubsection{2}

\section{Local Gradient Constraint}

In the slope constrained optimization (Petersson \& Sigmund, 1998), restrictions in the gradient are added to the optimum topology design problem. The idea is to restrict the partial derivatives.

This method allows controlling the minimum length scale based on two adjacent elements and one can choose the parameter control based on the element size (da Senhora, 2017). Nevertheless, it introduces too many extra constraints which slow down the optimization process and is, in general, considered unfeasible in practical cases.

\subsection{2}

\section{Linear Filter}

Filters can deal with checkerboards and mesh-dependency. Mainly, two filters have been used: the sensitivity and the design variable filter.

The sensitivity filter modifies the derivatives in a heuristic way, thus there is no mathematical proof. Since these derivatives are not consistent with the problem formulation, it cannot be established what is the optimization 
problem to be solved. But, Bendsøe \& Sigmund (2003) point out it has shown good results, specially to ensure mesh-independency results.

Considering the theoretical basis for the sensitivity filter still not yet understood the design variable filter (Bruns \& Tortorelli, 2001) appear as an alternative. Also know as the density filter, it acts directly modifying the densities of a certain element $z_{e}$, based on weighted average of the element densities in a defined neighborhood. It allows local control on material distribution and ensures a smooth transition of the density field.

For the discrete case and the design variable $\bar{z}_{e}$ located in the centroid of element $e$, the filter is

$$
y_{e}=\frac{\sum_{i \in N_{e}} w_{i}\left(\bar{z}_{e}, \bar{z}_{i}\right) z_{i}}{\sum_{i \in N_{e}} w_{i}\left(\bar{z}_{e}, \bar{z}_{i}\right)}
$$

where $y_{e}$ is the filtered design variable, $N_{e}$ is the number of elements in the domain $\Omega$ and $\bar{z}_{i}$ is the location of the $i$ th design variable. The weight distribution $w$ is stated as

$$
w_{i}\left(\bar{z}_{e}, \bar{z}_{i}\right)=\max \left(1-\frac{d_{i}}{r_{\min }}, 0\right),
$$

for $d_{i}$ being the distance from element $e$ to element $i$, that is, $d_{i}=\left\|\bar{z}_{e}-\bar{z}_{i}\right\|$, and $r_{\min }$ the filter radius, which is independent of the mesh.

Basically, a loop is scanned by checking whether $\bar{z}_{i}$ is within the radius $r_{\text {min }}$ defined by $\bar{z}_{e}$. Values greater than or equal to $r_{\text {min }}$ are null and the smaller ones are aggregated according to the weight $w_{i}$ in Equation (3.5), that works as a convolution operator.

The Equation (3.4) can be written in the matrix form

$$
\boldsymbol{y}=\boldsymbol{M z}
$$

on what $\boldsymbol{z}$ is the vector of design variables, $\boldsymbol{y}$ is the corresponded "filtered" vector and $\boldsymbol{M}$ is the matrix of weighted indices defined by

$$
M_{e i}=\frac{w_{i}\left(\bar{z}_{e}, \bar{z}_{i}\right)}{\sum_{i \in N_{e}} w_{i}\left(\bar{z}_{e}, \bar{z}_{i}\right)}
$$

This filter is also known as linear hat kernel since the weight function decays linearly with the distance from element $e$ : it has magnitude 1 at element 
centroid to 0 at $r_{\min }$ similar to a birthday party hat, as illustrated in Figure 3.7. Other weight distributions can be used, such as parabolic and Gaussian, but this technique will no longer remember this hat.

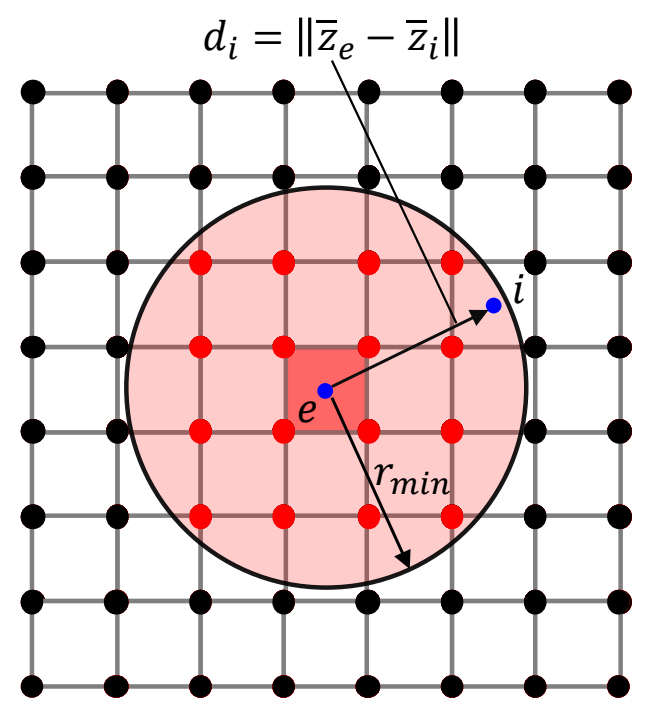

3.7(a): Top view.

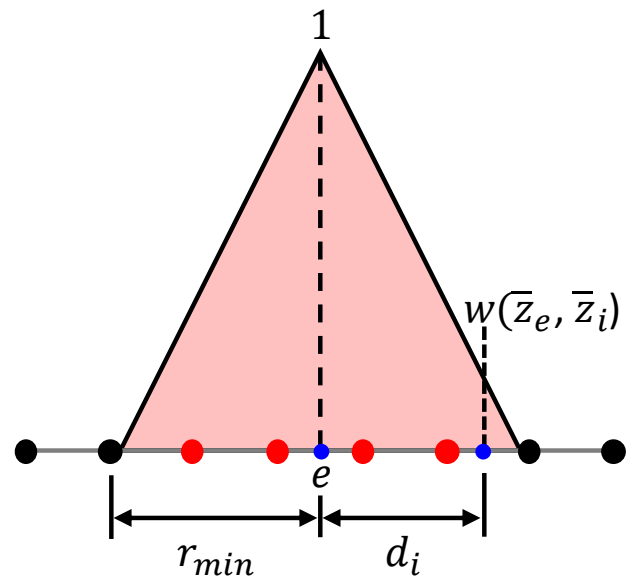

3.7(b): Frontal view.

Figure 3.7: Two-dimensional linear density filter: (a) application in a regular quadrilateral element mesh; (b) conical form alike to a birthday party hat.

One drawback of these filtering methods has been the transition regions between solids and voids in the contours of the optimal geometry. These intermediate densities though can be alleviated using nonlinear projection techniques or removing the filter effect after the optimization process has ended. 


\subsection{3}

\section{Nonlinear Projection Techniques}

Linear filters are inevitably submitted to fading effect that occurs along the edges of structural members. Guest et al. (2004) stated that this phenomenon is inherent to linear projections schemes and cannot be prevented through penalization methods. As emphasized by Talischi et al. (2012b), nonlinear projection functions can be combined to SIMP scheme to modify the material interpolation function. Then, this combination can be applied to the optimization procedure in such way that it deals with grays in the boundaries of structural members. Here, two of them are presented: exponential and tanh Heavisidebased functions.

\subsubsection{1}

\section{Exponential Projection Function}

The exponential projection function gives a continuous and smooth transition between solids and voids. It was introduced by Guest et al. (2004) to reduce gray scales on the edge of structural parts after the filtering process defined in Equation (3.4). In fact, it controls the minimum length scale of structural elements, that is, this function allows to control the size of solid members in the design domain. Being $\beta_{1}$ a parameter dictating this transition, the exponential function is

$$
g\left(y_{e}\right)=1-e^{-\beta_{1} y_{e}}+y_{e} e^{-\beta_{1}}
$$

where the term $y_{e}$ is the "filtered" design variable of an element $e$. This value can be conditioning in an array of projected densities $g(\boldsymbol{y})$. Doing that and recalling $\boldsymbol{y} \in[0,1]$, this array is penalized by SIMP, which can be rewritten in the form of:

$$
\tilde{\boldsymbol{x}}(\boldsymbol{y})=\epsilon+(1-\epsilon)(g(\boldsymbol{y}))^{p}, \quad \text { for } p \geqslant 1
$$

The projection function is linear for $\beta_{1}=0$ and approaches the Heaviside step function when $\beta_{1} \rightarrow \infty$. For the element $e$, Figure 3.8 demonstrates its behavior for different values of $\beta_{1}$.

Since many problems are solved using continuation, $\beta_{1}$ parameter is raised as the penalty exponent $p$ increases, until the fading areas are eliminated. For the exponential function, Guest et al. (2004) recommend starting with a low value of $\beta_{1}$, for example $\beta_{1}=1$, to reduce the probability of converging 
to a local minimum. This function, however, is susceptible to generate hinges, i.e., node-to-node connections.

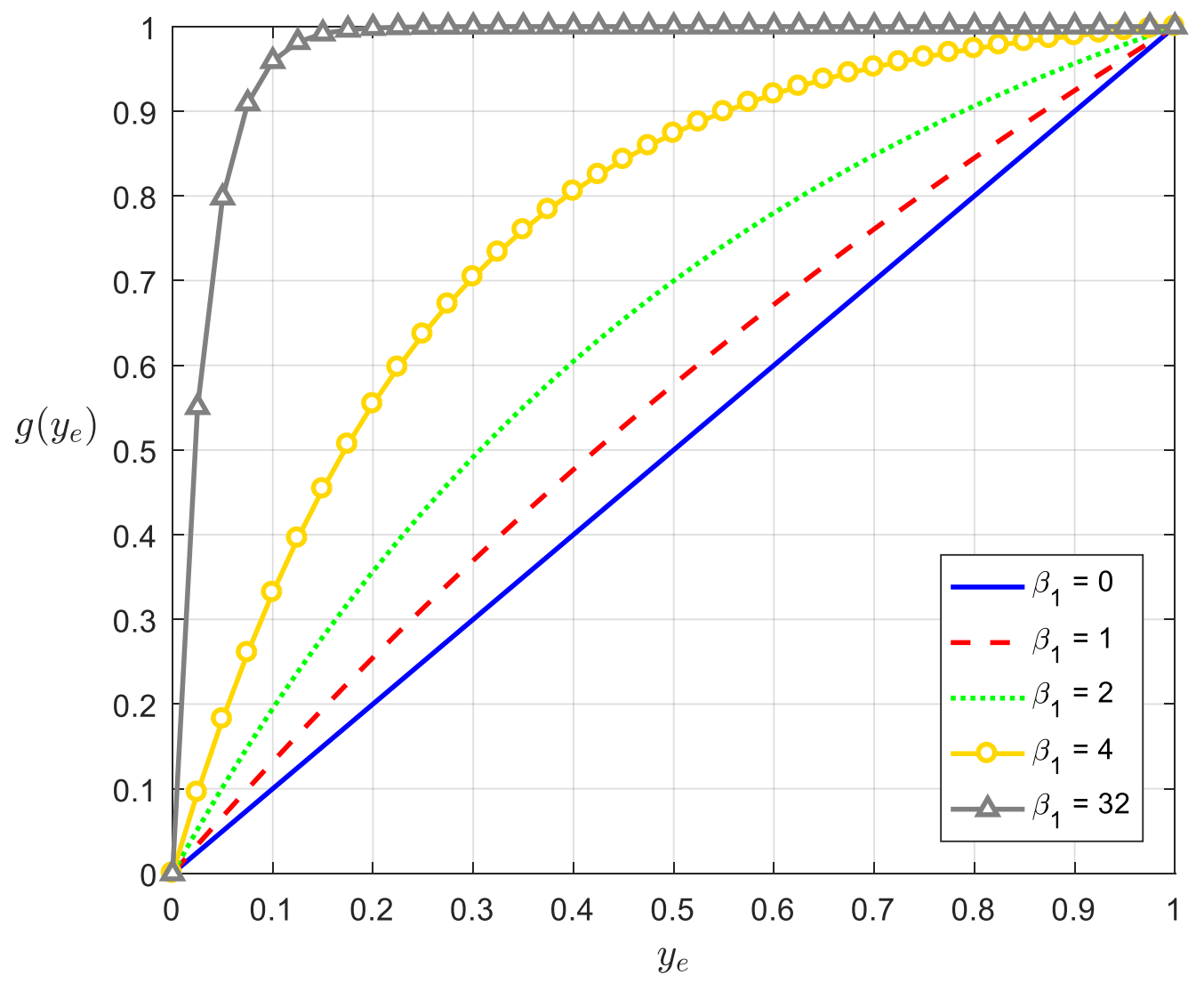

Figure 3.8: Regularized exponential function for several magnitudes of $\beta_{1}$.

\subsubsection{2}

\section{Hyperbolic Tangent (tanh) Projection Function}

Another nonlinear Heaviside-based projection function was studied by Wang et al. (2011):

$$
g\left(y_{e}\right)=\frac{\tanh \left(\beta_{2} \eta\right)+\tanh \left(\beta_{2}\left(y_{e}-\eta\right)\right)}{\tanh \left(\beta_{2} \eta\right)+\tanh \left(\beta_{2}(1-\eta)\right)}
$$

The variable $\eta$ is kept constant, $\eta=0.5$ as recommended by Wang et al. (2014), and $\beta_{2}$, responsible for the sharpness of the projection, is doubled every 10 iterations starting at 4 and ending at 64. It is easy to see from Equation (3.9) and Figure 3.9 that $\beta_{2}=1$ represents the linear behavior and the higher $\beta_{2}$, the closer it gets to Heaviside's function.

As procedure for the exponential function, an array of $g\left(y_{e}\right), g(\boldsymbol{y})$, can be combined with SIMP method as in Equation (3.8). In some analysis conducted in this thesis, this function was implemented alongside SIMP method acting 
when the penalization exponent $p=3$, so it will not influence on the behavior of low-stiffness elements.

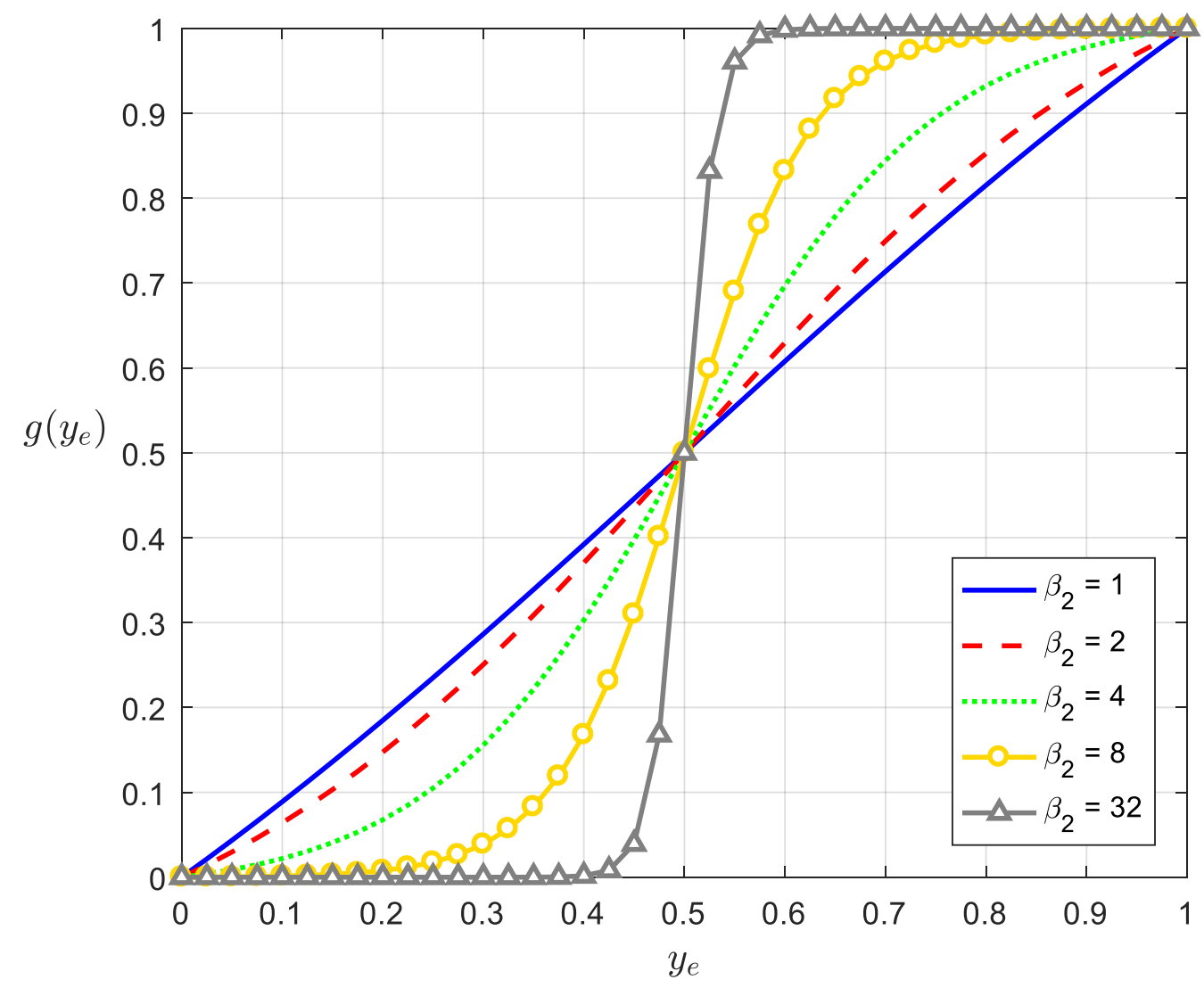

Figure 3.9: Hyperbolic tangent function for several magnitudes of $\beta_{2}$. 


\section{4 \\ Topology Optimization Under Geometric Nonlinearity}

\section{1}

\section{Introduction}

This chapter is divided into two parts: optimization problem and nonlinear methods. In the former, the "end-compliance" minimization problem is formalized, as well as the interpolation scheme - applied to deals with the large distortions in low-stiffness elements within the domain - and the sensitivity analysis, fundamentally necessary for gradient-based optimizers. In the latter, the nonlinear solution methods of load control, arc-length and generalized displacement control are reviewed. Those are formulated according to an unified library discussed by Leon et al. (2011). At the end, a flowchart for the optimization procedure is presented.

\section{2}

\section{Optimization Problem}

The studied optimization problem is the minimization of "end-compliance" $F_{c}$ defined by:

$$
\begin{array}{ll}
\min _{\boldsymbol{z}} & F_{c}=\boldsymbol{l}^{T} \boldsymbol{u} \\
\text { s.t.: } & \sum_{e=1}^{N_{e}} \frac{V\left(z_{e}\right)}{V_{0}}-V_{\text {frac }} \leqslant 0 \\
& 0 \leqslant \boldsymbol{z} \leqslant 1 \\
\text { with } & \boldsymbol{r}(\boldsymbol{z}, \boldsymbol{u})=\boldsymbol{l}-\boldsymbol{f}(\boldsymbol{z}, \boldsymbol{u}) \approx \mathbf{0} .
\end{array}
$$

The objective is defined by Equation (4.1a), where $\boldsymbol{l}, \boldsymbol{f}$ and $\boldsymbol{u}$ are, respectively, vectors of external and internal forces and displacements.

The amount of material is controlled by the volume fraction $V_{\text {frac }}$, representing the ratio between the final and initial volume: $V_{\text {frac }}=V_{f} / V_{0}$. This ratio controls the percentage of maximum material allowed in the final 
topology. The basic definition for the volume restriction is given as function of the density $\rho$ by

$$
\int_{\Omega} \rho \mathrm{d} V \leqslant V_{f}
$$

In optimization problem this restriction is discretized by Equation (4.1b) for $N_{e}$ elements in the design domain $\Omega . V\left(z_{e}\right)$ corresponds to the actual volume of the design variable $z_{e}$.

Equation (4.1c) limits the range of the design variable $\boldsymbol{z}$. The difference between external and internal forces, indicated in Equation (4.1d), corresponds to the structural equilibrium in which $\boldsymbol{r}$ is the residual force vector. In static or quasi-static analysis this expression must be zero.

\section{3}

\section{Modeling of Void Regions}

To reduce the convergence issues in geometrically nonlinear topology optimization routine, Pajot (2006) proposed a simply interpolation scheme. As the source of the numerical instabilities are associated to large displacements, the concept of this method is based on remodeling the kinematic relations (geometric nonlinearities). To do so, the element residual force $\boldsymbol{r}_{e}$ is written as a combination of linear and nonlinear terms:

$$
\boldsymbol{r}_{e}=\gamma_{e}^{h} \boldsymbol{r}_{e}^{N L}+\left(1-\gamma_{e}^{h}\right) \boldsymbol{r}_{e}^{L}, \quad 0 \leqslant \gamma_{e}^{h} \leqslant 1
$$

for $\gamma_{e}^{h}$ being the homotopic parameter and

$$
\begin{gathered}
\boldsymbol{r}_{e}^{L}=\boldsymbol{l}_{e}-\boldsymbol{f}_{e}^{L} \\
\boldsymbol{r}_{e}^{N L}=\boldsymbol{l}_{e}-\boldsymbol{f}_{e}^{N L},
\end{gathered}
$$

where $\boldsymbol{l}_{e}$ is the element external force vector and $\boldsymbol{f}_{e}^{L}$ and $\boldsymbol{f}_{e}^{N L}$ are, respectively, the linear and nonlinear terms of the element internal force vector.

Nevertheless, Wang et al. (2014) observed, when elements undergo large deformations, the approach of Equation (4.3) can still result in numerical instabilities for $0<\gamma_{e}^{h}<1$. As consequence, the authors proposed a different interpolation approach called energy interpolation scheme. The element elastic energy is interpolated between the large deformation theory and the small one. 
Ideally, if modeled by sufficiently low density, void elements do not influence the solid (structural) parts of the domain $\Omega$. With this concept in mind, lowdensity regions can be modeled according to linear strain energy theory and the structural regions by the nonlinear concepts of strain energy.

For the discrete case, this energy interpolation scheme is conceptualized as

$$
\phi_{e}\left(\boldsymbol{u}_{e}\right)=\left[\phi\left(\gamma_{e} \boldsymbol{u}_{e}\right)-\phi_{L}\left(\gamma_{e} \boldsymbol{u}_{e}\right)+\phi_{L}\left(\boldsymbol{u}_{e}\right)\right] E_{0 e}
$$

where $E_{0 e}$ and $\boldsymbol{u}_{e}$ are, respectively, the Young's modulus and the vector of displacements of element $e$. The parameter $\phi$ is the stored elastic energy density function for the base material with unit Young's modulus and $\phi_{L}$ is the stored elastic energy density under small deformations also with $E_{0 e}=1$. The element behavior is controlled by the interpolation factor $\gamma_{e}$, which assumes the value of 0 for voids or 1 for solids.

In this thesis the Pajot's formulation is combined with the interpolation factor described by Wang et al. (2014). Therefore, the residual is

$$
\begin{aligned}
\boldsymbol{r}_{e} & =\gamma_{e} \boldsymbol{r}_{e}^{N L}+\left(1-\gamma_{e}\right) \boldsymbol{r}_{e}^{L} \\
& =\gamma_{e}\left(\boldsymbol{l}_{e}-\boldsymbol{f}_{e}^{N L}\right)+\left(1-\gamma_{e}\right)\left(\boldsymbol{l}_{e}-\boldsymbol{f}_{e}^{L}\right) \\
& =\gamma_{e} \boldsymbol{l}_{e}-\gamma_{e} \boldsymbol{f}_{e}^{N L}+\boldsymbol{l}_{e}-\boldsymbol{f}_{e}^{L}-\gamma_{e} \boldsymbol{l}_{e}+\gamma_{e} \boldsymbol{f}_{e}^{L} \\
& =\boldsymbol{l}_{e}-\left(\gamma_{e} \boldsymbol{f}_{e}^{N L}+\left(1-\gamma_{e}\right) \boldsymbol{f}_{e}^{L}\right)
\end{aligned}
$$

The tangent stiffness matrix is derived directly from Equation (4.7):

$$
\begin{aligned}
\frac{\partial \boldsymbol{r}_{e}}{\partial \boldsymbol{u}} & =\frac{\partial}{\partial \boldsymbol{u}}\left(\boldsymbol{l}_{e}-\boldsymbol{f}_{e}\right) \\
& =-\left(\gamma_{e} \frac{\partial \boldsymbol{f}_{e}^{N L}}{\partial \boldsymbol{u}}+\left(1-\gamma_{e}\right) \frac{\partial \boldsymbol{f}_{e}^{L}}{\partial \boldsymbol{u}}\right) \\
& =-\left(\gamma_{e} \boldsymbol{K}_{e}^{N L}+\left(1-\gamma_{e}\right) \boldsymbol{K}_{e}^{L}\right) .
\end{aligned}
$$

There is only one modification to be added in Equations (4.7) and (4.8): the design variable $\tilde{x}_{e}$, responsible to assign existence of material (or not) in the 
design domain (see Sections 3.2.1 and 3.4.2). Then, the interpolation procedure is defined by

$$
\begin{gathered}
\boldsymbol{f}_{e}=\tilde{x}_{e}\left[\gamma_{e} \boldsymbol{f}_{e}^{N L}+\left(1-\gamma_{e}\right) \boldsymbol{f}_{e}^{L}\right] \\
\boldsymbol{K}_{e}=\tilde{x}_{e}\left[\gamma_{e} \boldsymbol{K}_{e}^{N L}+\left(1-\gamma_{e}\right) \boldsymbol{K}_{e}^{L}\right],
\end{gathered}
$$

for $\boldsymbol{K}_{e}$ and $\boldsymbol{f}_{e}$ being, respectively, the tangent stiffness matrix and the internal force vector of eth element. $\boldsymbol{K}_{e}^{L}, \boldsymbol{K}_{e}^{N L}, \boldsymbol{f}_{e}^{L}$ and $\boldsymbol{f}_{e}^{N L}$ are presented in Section 4.3.1.

On the implemented interpolation method, to associate linear behavior on small "densities" $\tilde{x}_{e}$, the interpolation factor $\gamma_{e}$ is

$$
\gamma_{e}\left(\tilde{x}_{e}\right)=\frac{\tanh (\alpha \bar{\rho})+\tanh \left(\alpha\left(\tilde{x}_{e}-\bar{\rho}\right)\right)}{\tanh (\alpha \bar{\rho})+\tanh (\alpha(1-\bar{\rho}))}
$$

In Equation (4.11), $\alpha$ controls the sharpness of the interpolation factor and $\bar{\rho}$ is a threshold to determine the element behavior. The parameter $\alpha$ must assume a higher value to guaranty a smooth transition between linear and nonlinear cases. The concerning variable is $\bar{\rho}$ : a low value, $\bar{\rho} \rightarrow 0$, imposes a linear behavior on the elements, what does not reproduce the mechanics of a large deformation problem. Besides, for high values $(\bar{\rho} \rightarrow 1)$, the void elements assume large displacements due to nonlinear behavior and the distortion associated to them will retard or result in non-convergence of the topology optimization process. Based on numerical experiments, the best pair of values capable of reducing the distortion of void regions is

$$
\alpha=500 ; \quad \bar{\rho}=0.01,
$$

as suggested by Wang et al. (2014) in the reference paper.

\subsection{1}

\section{Tangent Stiffness Matrices and Internal Force Vectors}

The element tangent stiffness matrix $\boldsymbol{K}_{e}$ under small deformation theory is

$$
\boldsymbol{K}_{e}^{L}=\int_{\Omega_{e}} \boldsymbol{B}_{L 0}^{T} \boldsymbol{D}^{0} \boldsymbol{B}_{L 0} \mathrm{~d} V
$$

and for large deformations it is 


$$
\begin{aligned}
\boldsymbol{K}_{e}^{N L} & =\boldsymbol{K}_{e}^{L}+\boldsymbol{K}_{e}^{D}+\boldsymbol{K}_{e}^{S} \\
& =\int_{\Omega_{e}}\left[\left(\boldsymbol{B}_{L 0}+\boldsymbol{B}_{L 1}\right)^{T} \boldsymbol{D}^{0}\left(\boldsymbol{B}_{L 0}+\boldsymbol{B}_{L 1}\right)+\boldsymbol{B}_{N L}^{T} \tilde{\boldsymbol{S}} \boldsymbol{B}_{N L}\right] \mathrm{d} V .
\end{aligned}
$$

The element internal force $\boldsymbol{f}_{e}$ for small deformations is

$$
\boldsymbol{f}_{e}^{L}=\boldsymbol{K}_{e}^{L} \boldsymbol{u}_{e}
$$

and for large deformations it is

$$
\boldsymbol{f}_{e}^{N L}=\int_{\Omega_{e}}\left(\boldsymbol{B}_{L 0}+\boldsymbol{B}_{L 1}\right)^{T} \hat{\boldsymbol{S}} \mathrm{d} V
$$

For further detail on these matrices and the finite element theory, one can go back to Section 2.3 or consult Bathe (2014), among other classical readings.

\subsection{2}

\section{C-shaped Benchmark Problem}

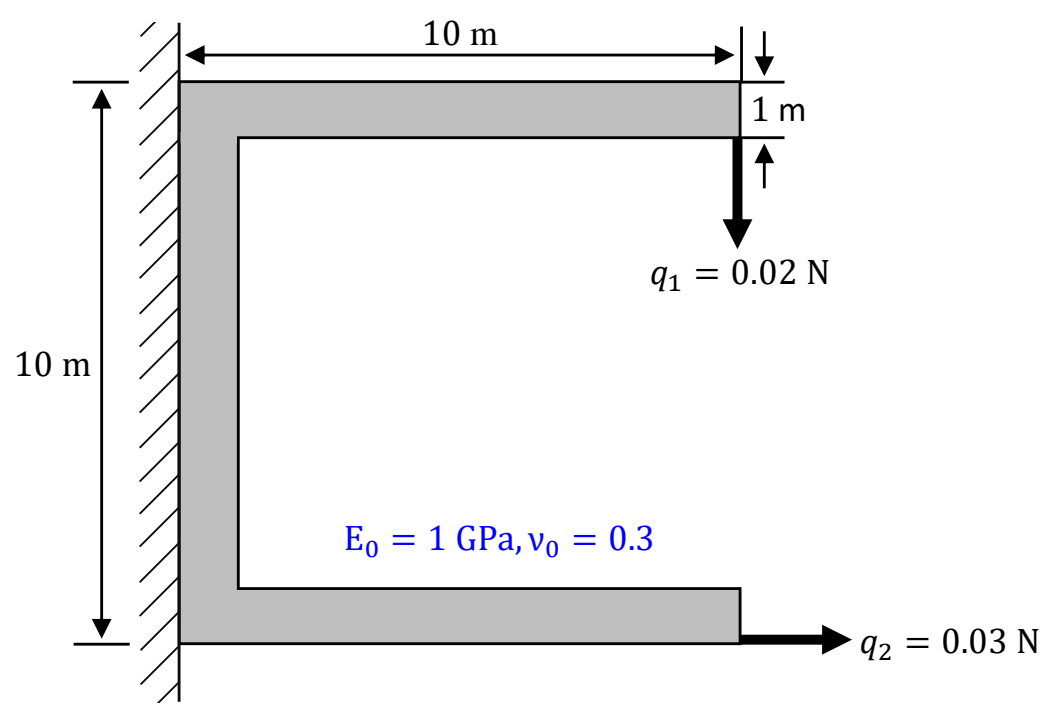

Figure 4.1: C-shape.

The C-shape is a benchmark example proposed by Yoon \& Kim (2005a) to study large distortions in void elements of the finite element mesh, which helps clarifying the idea and the benefits behind the interpolation technique in study. As shown in Figure 4.1, the C-shape is fixed on the left side and it is submitted to two different loads $q_{i}, i=1,2$. This geometry has unitary Young's modulus $E_{0}$ and Poisson's coefficient $\nu_{0}=0.3$. 
Figure 4.2(a) illustrates the C-shape geometry, discretized by Q4 elements, and Figure 4.2(b) displays the Euclidean norm of displacement $\|\boldsymbol{u}\|$ for a linear analysis. To represent the linear behavior, the magnitude of loads are set as $10 \%$ of those indicated in Figure 4.1 and a plane stress state (depth $d=1 \mathrm{~m}$ ) is assumed for both linear and nonlinear analysis conducted in this section.

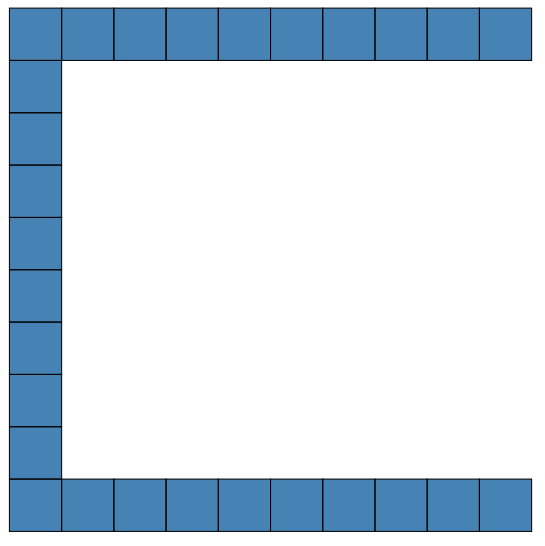

4.2(a): Geometry representation.

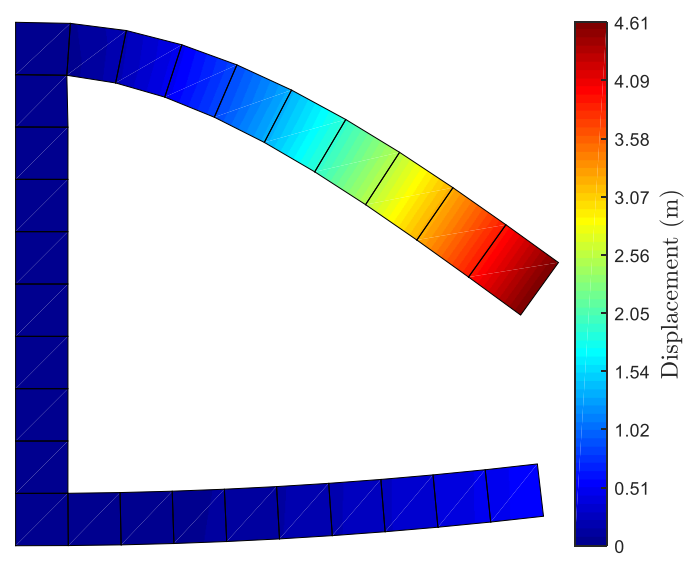

4.2(b): Deformation and displacement field.

Figure 4.2: C-shape geometry when modeling only solid elements.

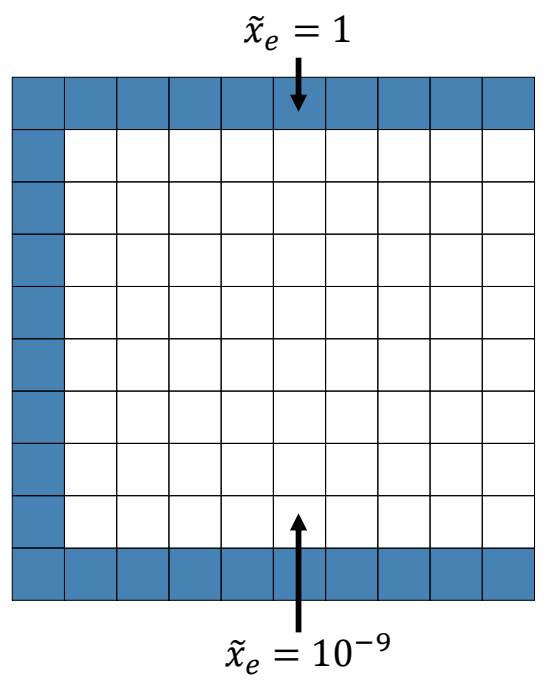

4.3(a): Geometry representation.

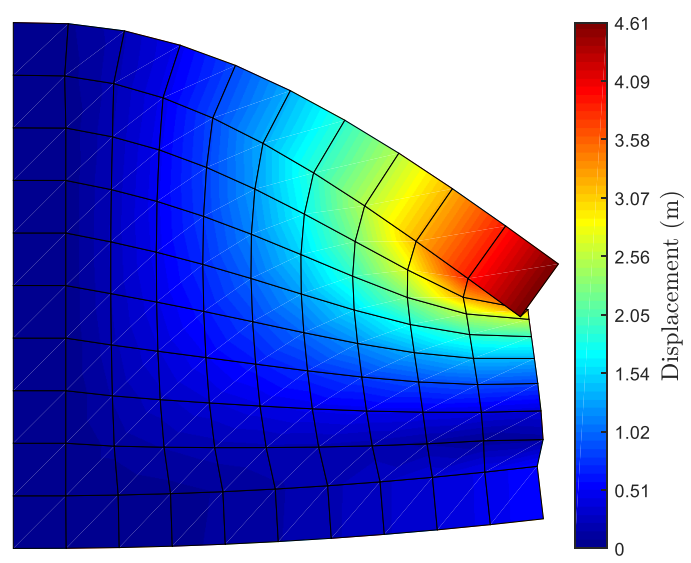

4.3(b): Deformation and displacement field.

Figure 4.3: C-shape linear analysis when modeling solid (light blue) and void (white) elements in a fictitious domain representation.

To investigate the difficulties imposed to the nonlinear solution methods, the C-shape is modeled using fictitious domain approach (Ramiere et al., 2007), cf. Figure 4.3(a). The domain is discretized by $10 \times 10$ quadrilateral elements. The idea of using this representation, here, is to reproduce a possible result from topology optimization. Following this approach, the solid elements are 
modeled for a design variable $\tilde{x}_{e}=1$, while voids are represented by small values, e.g., $\tilde{x}_{e}=10^{-9}$. For the linear case, both the displacement field and the deformation of the solid regions in Figure 4.2(b) and its fictitious domain representation in Figure 4.3(b) are the same, which indicates that the behavior of the structural members does not depend on the way the voids are modeled.

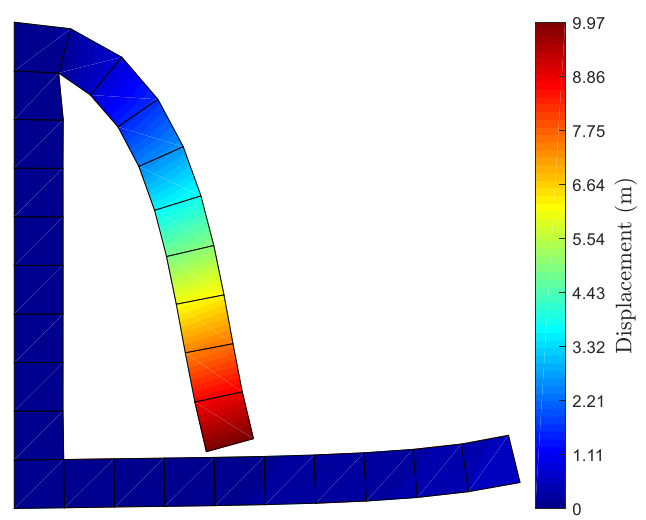

4.4(a): Modeling only solid elements by the large deformation theory.
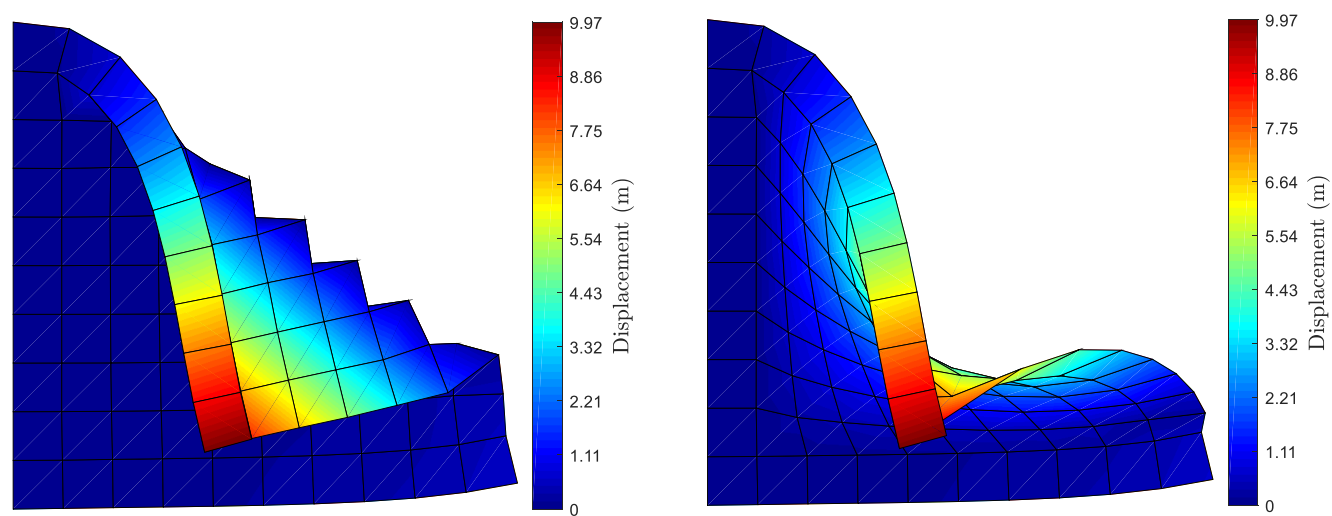

4.4(b): Modeling solids and voids by the large 4.4(c): Modeling solid elements by the large deformation theory. deformation theory and voids by the small one.

Figure 4.4: C-shape nonlinear analysis. Deformation and displacement field for the indicated cases.

Regarding the nonlinear case, Figure 4.4(a) illustrates the deformation for the simple C-shape - only the solids are modeled. Assuming the same domain of Figure 4.3(a), Figure 4.4(b) represents the deformation when the elements' bevavior are modeled considering the large deformation theory. In Figure 4.4(c) the elements' behavior are interpolated by means of the interpolation scheme defined in Equations (4.9) and (4.10).

In Figure 4.4(c) the voids have a smooth fashion deformation than those in Figure 4.4(b) and, despite they still inverted, this is not seen by the solver, due to the linear behavior attributed to them. The investigated interpolation method facilitates the convergence as it reduces the number of 
iterations taken by the nonlinear solution method. Without this interpolation approach the algorithm took 501 iterations in the Newton-Raphson method. In turn, interpolating the elements' behavior only 180 were needed. Thus, this interpolation technique required $36 \%$ of the iterations in the Newton-Raphson approach of those needed in the nonlinear case to converge.

\section{4}

\section{Sensitivity Analysis of the Objective}

Assuming the equilibrium has been reached, the residual force vector $\boldsymbol{r}$ is zero and the Equation (4.1a) can be rewritten in function of the adjoint vector $\boldsymbol{\psi}$ :

$$
F_{c}=\boldsymbol{l}^{T} \boldsymbol{u}+\boldsymbol{\psi}^{T} \boldsymbol{r}
$$

The external force $\boldsymbol{l}$ is independent on the (element) design variable $\tilde{x}_{e}$, but the residual force depends on both design variable and displacement, therefore the sensitivity of Equation (4.16) is given as

$$
\frac{d F_{c}}{d \tilde{x}_{e}}=\boldsymbol{l}^{T} \frac{\partial \boldsymbol{u}}{\partial \tilde{x}_{e}}+\boldsymbol{\psi}^{T}\left(\frac{\partial \boldsymbol{r}}{\partial \tilde{x}_{e}}+\frac{\partial \boldsymbol{r}}{\partial \boldsymbol{u}} \frac{\partial \boldsymbol{u}}{\partial \tilde{x}_{e}}\right)
$$

where $\partial \boldsymbol{r} / \partial \boldsymbol{u}$ is obtained by differentiating equation (4.1d) for a fix design variable,

$$
\frac{\partial \boldsymbol{r}}{\partial \boldsymbol{u}}=-\frac{\partial \boldsymbol{f}}{\partial \boldsymbol{u}}=-\boldsymbol{K}
$$

and isolating the term $\partial \boldsymbol{u} / \partial \tilde{x}_{e}$ in Equation (4.17) results in:

$$
\frac{d F_{c}}{d \tilde{x}_{e}}=\left(\boldsymbol{l}^{T}-\boldsymbol{\psi}^{T} \boldsymbol{K}\right) \frac{\partial \boldsymbol{u}}{\partial \tilde{x}_{e}}+\boldsymbol{\psi}^{T} \frac{\partial \boldsymbol{r}}{\partial \tilde{x}_{e}}
$$

Since the adjoint vector is arbitrary, it can be employed to eliminate the implicit response $\partial \boldsymbol{u} / \partial \tilde{x}_{e}$. To do so, the first term on the right-hand side of Equation (4.19) should vanishes and the natural choice for the adjoint vector is

$$
\boldsymbol{\psi}=\boldsymbol{K}^{-1} \boldsymbol{l}
$$

Substituting the nonlinear state equation of Equation (4.1d) into Equation (4.19), the sensitivity of the objective with respect to the design variable 
becomes

$$
\frac{d F_{c}}{d \tilde{x}_{e}}=-\psi^{T} \frac{\partial \boldsymbol{f}}{\partial \tilde{x}_{e}}
$$

The derivative of internal force $\boldsymbol{f}_{e}$ for the eth element is

$$
\frac{\partial \boldsymbol{f}_{e}}{\partial \tilde{x}_{e}}=\frac{\partial}{\partial \tilde{x}_{e}}\left[\tilde{x}_{e}\left(\gamma_{e} \boldsymbol{f}_{e}^{N L}+\left(1-\gamma_{e}\right) \boldsymbol{f}_{e}^{L}\right)\right]
$$

and, after few manipulations, one obtains

$$
\frac{\partial \boldsymbol{f}_{e}}{\partial \tilde{x}_{e}}=\gamma_{e} \boldsymbol{f}_{e}^{N L}+\left(1-\gamma_{e}\right) \boldsymbol{f}_{e}^{L}+\tilde{x}_{e} \frac{\partial \gamma_{e}}{\partial \tilde{x}_{e}}\left(\boldsymbol{f}_{e}^{N L}-\boldsymbol{f}_{e}^{L}\right)
$$

in which the derivative of the interpolation factor is

$$
\frac{\partial \gamma_{e}}{\partial \tilde{x}_{e}}=\frac{\alpha \operatorname{sech}^{2}\left(\alpha\left(\tilde{x}_{e}-\bar{\rho}\right)\right)}{\tanh (\alpha(1-\bar{\rho}))+\tanh (\alpha \bar{\rho})}
$$

Finally, the sensitivity of the residual force $\boldsymbol{r}$, with respect to the design variable $z_{e}$, is calculated by the following chain rule, taken on the $N_{e}$ elements in the mesh,

$$
\frac{\partial \boldsymbol{r}}{\partial z_{e}}=-\frac{\partial \boldsymbol{f}}{\partial z_{e}}=-\sum_{j=1}^{N_{e}}\left(\frac{\partial \boldsymbol{f}_{j}}{\partial \tilde{x}_{j}}+\frac{\partial \boldsymbol{f}_{j}}{\partial \gamma_{j}} \frac{\partial \gamma_{j}}{\partial \tilde{x}_{j}}\right) \frac{\partial \tilde{x}_{j}}{\partial y_{j}} \frac{\partial y_{j}}{\partial z_{e}}
$$

where is immediately to observe that the term in parenthesis corresponds to the expression in Equation (4.23) and

$$
\frac{\partial y_{a}}{\partial z_{b}}=M_{a b}
$$

as stated in Equation (3.6). The derivative of $\tilde{x}_{j}$ with respect to $y_{j}$ depends on the design variable penalty method and whether or not projection function is applied. That is,

$$
\frac{\partial \tilde{x}_{j}}{\partial y_{j}}= \begin{cases}p(1-\epsilon) y_{j}^{p-1} & \text { for SIMP method } \\ p(1-\epsilon) g_{1}\left(y_{j}\right)^{p-1} \frac{\partial g_{1}}{\partial y_{j}} & \text { for exponential projection function } \\ p(1-\epsilon) g_{2}\left(y_{j}\right)^{p-1} \frac{\partial g_{2}}{\partial y_{j}} & \text { for tanh projection function }\end{cases}
$$


with:

$$
\begin{gathered}
g_{1}\left(y_{j}\right)=1-e^{-\beta_{1} y_{j}}+y_{j} e^{-\beta_{1}} \\
\frac{\partial g_{1}}{\partial y_{j}}=\beta_{1} e^{-\beta_{1} y_{j}}+e^{-\beta_{1}} \\
g_{2}\left(y_{j}\right)=\frac{\tanh \left(\beta_{2} \eta\right)+\tanh \left(\beta_{2}\left(y_{j}-\eta\right)\right)}{\tanh \left(\beta_{2} \eta\right)+\tanh \left(\beta_{2}(1-\eta)\right)} \\
\frac{\partial g_{2}}{\partial y_{j}}=\frac{-\beta_{2}\left(\tanh \left(\beta_{2}\left(\eta-y_{j}\right)\right)^{2}-1\right)}{\tanh \left(\beta_{2} \eta\right)-\tanh \left(\beta_{2}(\eta-1)\right)} .
\end{gathered}
$$

The comparison between the analytical sensitivity, discussed above, and the sensitivity calculated via finite differences is presented in appendix B.

\section{5}

\section{Nonlinear Solution Schemes}

When solving nonlinear problems the algorithm has to be able to trace complex equilibrium paths. These paths feature critical points, in which structure loses stability.

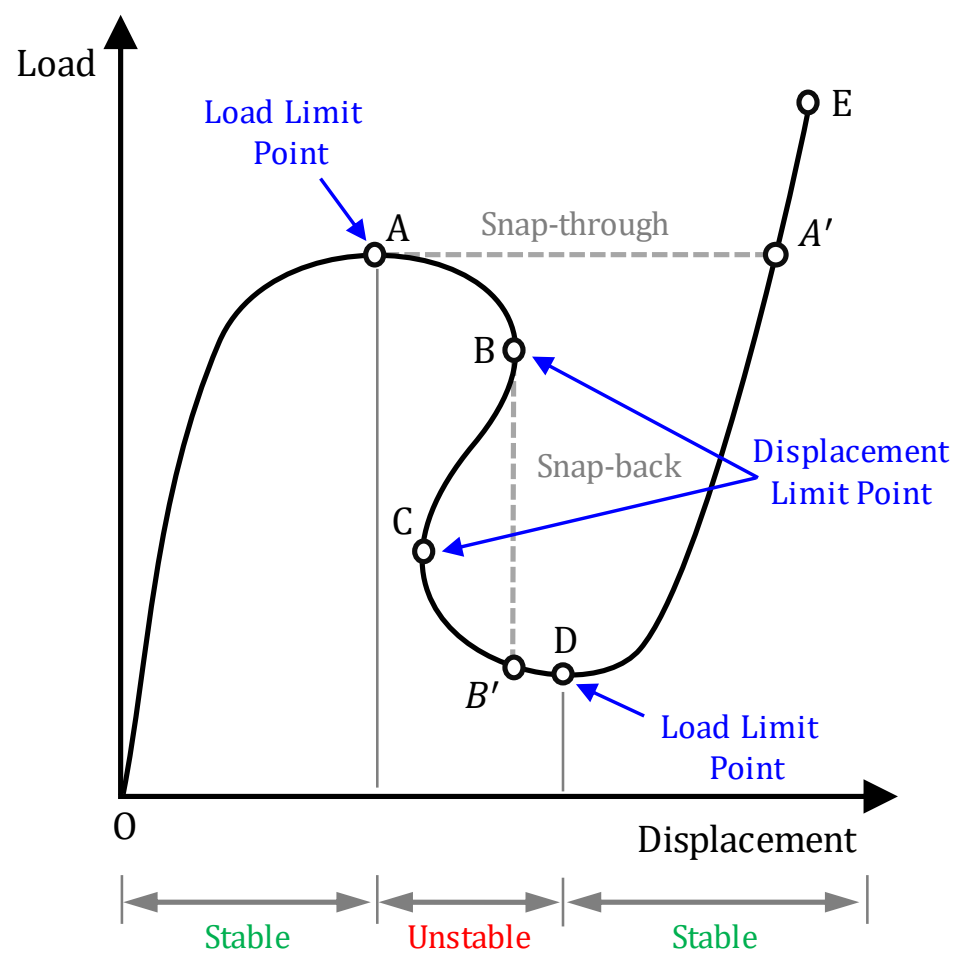

Figure 4.5: Representative equilibrium path.

Consider the schematic path illustrated in Figure 4.5. Load limit points occur at local maxima or minima. In one-dimension problem, it is characterized 
by a horizontal tangent (points $A$ e $D$ ); displacement limit points occur in verticals tangents (points $C$ e $C$ ). Nonlinear schemes which fails to trace beyond load limit points present the snap-through phenomenon. The ones capable of passing the displacement limit points are said to capture snap-back behavior.

Equilibrium curves can be traced by applying either a purely incremental procedure or an incremental-iterative procedure. In the purely incremental method, the load is applied in relatively small steps. It is assumed that each incremental step exhibits linear behavior. Despite the ease of implementation, this method tends to diverge from the real path, since it does not ensure equilibrium at every solution step.

In the incremental-iterative method, a series of iterations are performed at each incremental step until a certain convergence criterion is satisfied. Among many criteria in literature, a simple one is adopted: $\|\delta \boldsymbol{u}\| \leqslant C O N V$, where $C O N V=10^{-6}$ is the specified convergence tolerance. This convergence checking, applied in small increments, assures that the correct equilibrium path is traced. The nonlinear solution schemes discussed in this thesis are incremental and iterative, cf. Figure 4.6.

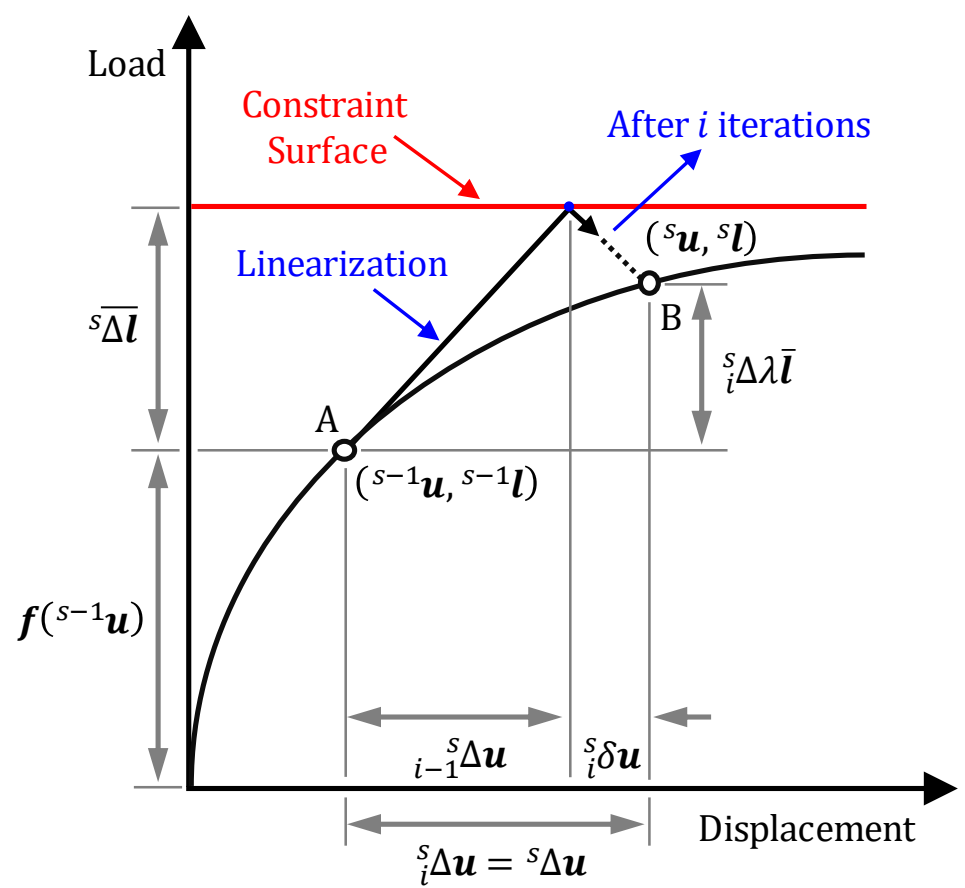

Figure 4.6: Incremental-iterative procedure.

To represent the incremental-iterative solution the following notation is proposed:

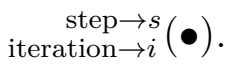


The left superscript $s$ represents the incremental step (or, simply, step or increment) and left subscript $i$ is the iteration. This representation allows the reader to distinguish it from the index notation, often used in Continuum Mechanics, and from the analysis taken on elements - denoted by the right subscript $e$.

In nonlinear problems, the internal forces ${ }_{i}^{s} \boldsymbol{f}$ are functions of displacements ${ }_{i}^{s} \boldsymbol{u}$ and so, ${ }_{i}^{s} \boldsymbol{f} \equiv \boldsymbol{f}\left({ }_{i}^{s} \boldsymbol{u}\right)$. These forces are not necessarily in equilibrium with the external ones, so an unbalanced or residual force ${ }_{i}^{s} \boldsymbol{r}={ }_{i}^{s} \boldsymbol{l}-\boldsymbol{f}\left({ }_{i}^{s} \boldsymbol{u}\right)$ is generated.

The displacements and external forces at step $s$ are computed by adding the contributions from the previously converged step, $s-1$, and the incremental update at the $i$ th iteration of the $s$ th incremental step:

$$
\begin{gathered}
{ }_{i}^{s} \boldsymbol{u}={ }^{s-1} \boldsymbol{u}+{ }_{i}^{s} \Delta \boldsymbol{u} \\
{ }_{i}^{s} \boldsymbol{l}={ }^{s-1} \boldsymbol{l}+{ }_{i}^{s} \Delta \boldsymbol{l} .
\end{gathered}
$$

In turn, the incremental update vectors of displacement and external force at step $s$ are reckoned by adding, respectively, the contributions on the previous $(i-1)$ th iteration and the iterative updates from the current $i$ th iteration. Thus:

$$
\begin{gathered}
{ }_{i}^{s} \Delta \boldsymbol{u}={ }_{i-1}^{s} \Delta \boldsymbol{u}+{ }_{i}^{s} \delta \boldsymbol{u} \\
{ }_{i}^{s} \Delta \boldsymbol{l}={ }_{i-1}^{s} \Delta \boldsymbol{l}+{ }_{i}^{s} \delta \boldsymbol{l},
\end{gathered}
$$

where ${ }_{i-1}^{s} \Delta \boldsymbol{u}=0$ for $i=1$, since there are no previous iterations at the first iteration of sth step. The vectors ${ }_{i}^{s} \delta \boldsymbol{u}$ and ${ }_{i}^{s} \delta \boldsymbol{l}$ are, respectively, the iterative displacement and force vectors at iteration $i$ and incremental step $s$.

Recalling Equations (4.31) to (4.34), the residual force can be written as

$$
{ }_{i}^{s} \boldsymbol{r}={ }^{s-1} \boldsymbol{l}+{ }_{i}^{s} \Delta \boldsymbol{l}-\boldsymbol{f}\left({ }^{s-1} \boldsymbol{u}+{ }_{i}^{s} \Delta \boldsymbol{u}\right)
$$

and the equilibrium equation for nonlinear systems is:

$$
{ }_{i-1}^{s} \boldsymbol{K}_{i}^{s} \delta \boldsymbol{u}={ }_{i}^{s} \boldsymbol{l}-{ }_{i-1}^{s} \boldsymbol{f}
$$

The tangent stiffness matrix ${ }_{i-1}^{s} \boldsymbol{K}$ can be evaluated by either the stan- 
dard or the modified method. In the former, the stiffness is evaluated at each iteration for every step. In the latter, the tangent matrix is calculated only at the first iteration of the sth step, i.e., ${ }_{i-1}^{s} \boldsymbol{K}={ }_{0}^{s} \boldsymbol{K}$.

In the finite element analysis, building the tangent stiffness matrix and solving the matrix equation are computationally expensive tasks. The modified method appears as an option to reduce the costs since it avoids the need to reformulate the tangent stiffness matrix at each iteration. In addition, this can also reduce the computational time required for solving the matrix equation. On the other hand, this technique usually presents a slower (linear) convergence, while standard method converges quadratically. As here the main objective is not about the algorithm performance, no further discussion is presented and the standard method is applied in all analyses.

\subsection{1}

\section{Unified Library}

By unified library, one can understand it as an unique set of equations in which certain parameters will be adjusted according to the required nonlinear solution method. Among many libraries proposed in literature, here, the nonlinear methods are formulated according to the unified solution discussed by Leon et al. (2011).

In the unified library an extra dimension is introduced in the solution space. The load parameter ${ }_{i}^{s} \delta \lambda$ is brought into Equation (4.34) by replacing ${ }_{i}^{s} \delta \boldsymbol{l}$ with ${ }_{i}^{s} \delta \lambda \overline{\boldsymbol{l}}$, in which $\overline{\boldsymbol{l}}$ is the reference force vector. Ergo, from Equation (4.32), the external force can be written as:

$$
{ }_{i}^{s} \boldsymbol{l}={ }^{s-1} \boldsymbol{l}+{ }_{i-1}^{s} \Delta \boldsymbol{l}+{ }_{i}^{s} \delta \lambda \overline{\boldsymbol{l}} .
$$

Substituting Equation (4.37) into (4.36) and comparing this result to Equation (4.35) leads to the system of governing equations:

$$
{ }_{i-1}^{s} \boldsymbol{K}_{i}^{s} \delta \boldsymbol{u}={ }_{i-1}^{s} \boldsymbol{r}+{ }_{i}^{s} \delta \lambda \overline{\boldsymbol{l}} .
$$

The above expression has $n$ variables, referred to the $n$ degree's of freedom ${ }_{i}^{s} \delta \boldsymbol{u}$, plus the load parameter ${ }_{i}^{s} \delta \lambda$. Therefore, this system has $n+1$ unknowns, but only $n$ equations. To solve it, an additional constraint is added to the system. The nonlinear solution schemes differs only in the use of different control parameters to set the incremental steps and perform the iterations. Thus, this restriction can be expressed in terms of general parameters, which 
are adjusted according to the desired nonlinear scheme. Yang \& Kuo (1994) suggest to write this constraint equation in the form of

$$
{ }_{i}^{s} \boldsymbol{a} \cdot{ }_{i}^{s} \delta \boldsymbol{u}+{ }_{i}^{s} b{ }_{i}^{s} \delta \lambda={ }_{i}^{s} c,
$$

where the effectiveness and reliability of the nonlinear solution technique depend directly on the choice of the constants, ${ }_{i}^{s} \boldsymbol{a}$ and ${ }_{i}^{s} b$ and the increment parameter ${ }_{i}^{s} c$.

Equation (4.39) can be viewed as the constraint for an optimization problem. Leon et al. (2011) assembles this system in a matrix form:

$$
\left[\begin{array}{cc}
{ }_{i-1}^{s} \boldsymbol{K} & -\overline{\boldsymbol{l}} \\
\left({ }_{i}^{s} \boldsymbol{a}\right)^{T} & { }_{i}^{s} b
\end{array}\right]\left\{\begin{array}{c}
{ }_{i}^{s} \delta \boldsymbol{u} \\
{ }_{i}^{s} \delta \lambda
\end{array}\right\}=\left\{\begin{array}{c}
{ }_{i-1}^{s} \boldsymbol{r} \\
{ }_{i}^{s} c
\end{array}\right\}
$$

Such augmented system is no longer symmetric and the solution of it, in general, is impractical due to the high computational cost for both storage and efficiency. To overcome this problem, the iterative displacement vector is often decomposed into the terms ${ }_{i}^{s} \delta \boldsymbol{u}_{l}$ and ${ }_{i}^{s} \delta \boldsymbol{u}_{r}$,

$$
{ }_{i}^{s} \delta \boldsymbol{u}={ }_{i}^{s} \delta \lambda{ }_{i}^{s} \delta \boldsymbol{u}_{l}+{ }_{i}^{s} \delta \boldsymbol{u}_{r},
$$

and the Equation (4.38) turn into:

$$
\begin{gathered}
{ }_{i-1}^{s} \boldsymbol{K}_{i}^{s} \delta \boldsymbol{u}_{l}=\overline{\boldsymbol{l}} \\
{ }_{i-1}^{s} \boldsymbol{K}_{i}^{s} \delta \boldsymbol{u}_{r}={ }_{i-1}^{s} \boldsymbol{r} .
\end{gathered}
$$

One can observe this set of equations is mathematically equivalent to the expression in Equation (4.38). The stiffness matrix is the same in both expressions and it maintains its original properties, e.g., the symmetry.

To determine ${ }_{i}^{s} \delta \boldsymbol{u}$, the load parameter has to be calculated. This is easily done substituting Equation (4.41) into Equation (4.39):

$$
{ }_{i}^{s} \delta \lambda=\frac{{ }_{i}^{s} c-{ }_{i}^{s} \boldsymbol{a} \cdot{ }_{i}^{s} \delta \boldsymbol{u}_{r}}{{ }_{i}^{s} \boldsymbol{a} \cdot{ }_{i}^{s} \delta \boldsymbol{u}_{l}+{ }_{i}^{s} b} .
$$

Hereafter, by means of an unified library, three nonlinear solution schemes will be discussed: load control method, arc-length method and generalized displacement control method. The first and the third were indeed implemented 
in the nonlinear topology optimization code. The arc-length procedure is the one often available in commercial finite element softwares. Its response, investigated in $\mathrm{ANSYS}^{\circledR}$, was used to validate the nonlinear solution scheme in the Lee-Frame structure (see Appendix A).

\subsubsection{1}

\section{Load Control Method}

The Newton-Raphson Procedure (NRP) is one of the oldest and most popular iterative nonlinear method. Most of techniques for solving nonlinear equations are derived from this scheme. It directly imposes the load parameter ${ }_{i}^{s} \delta \lambda$ and because of that, the NRP is also known as Load Control Method (LCM). For each step $s$, the load parameter is computed in the first iteration, $i=1$, and held constant over the remaining iterations in the sth step, cf. Figure 4.7.

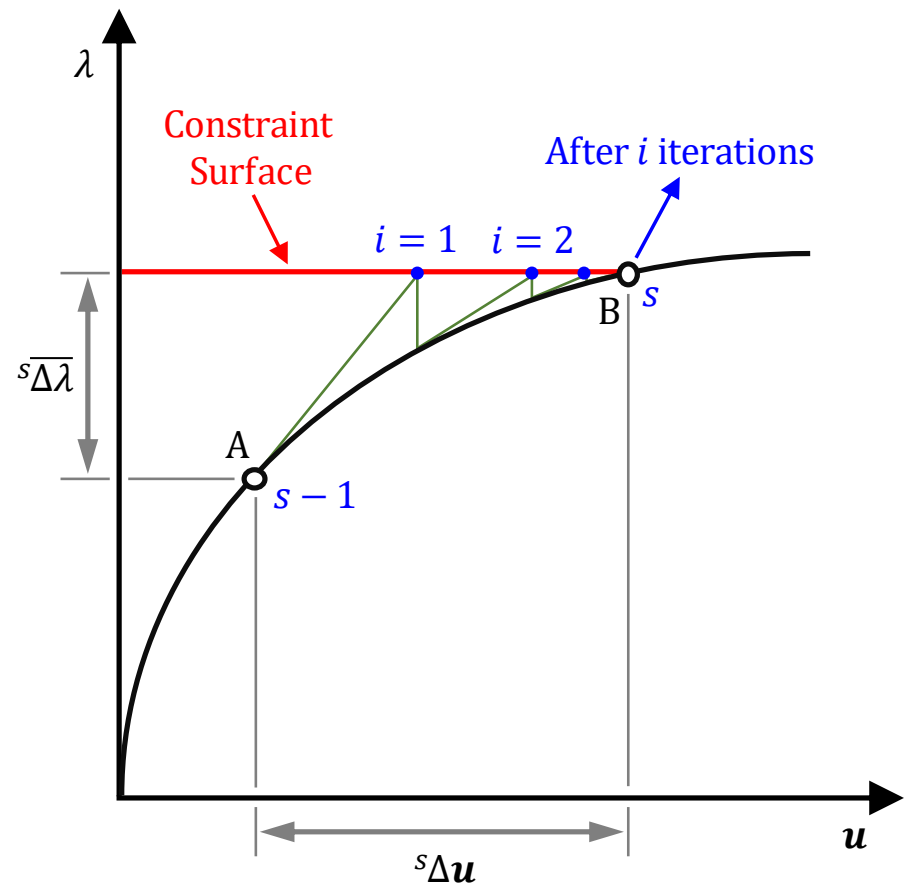

Figure 4.7: Load control method.

Looking at Equation (4.43), the unknown parameters of constraint Equation (4.39) can be defined as:

$$
\begin{gathered}
{ }_{i}^{s} \boldsymbol{a}=\mathbf{0} \\
{ }_{i}^{s} b=1 \\
{ }_{i}^{s} c={ }_{i}^{s} \delta \lambda= \begin{cases}{ }^{s} \overline{\Delta \lambda} & \text { for } i=1 \\
0 & \text { for } i \geqslant 2 .\end{cases}
\end{gathered}
$$


The term ${ }^{s} \overline{\Delta \lambda}$ is a prescribed initial load factor. This value can be interpreted as a percentage of the reference force $\overline{\boldsymbol{l}}$ and it does not have to be constant, i.e., its value can be changed according to the level of nonlinearity presented in the equilibrium path.

The total load factor ${ }_{i}^{s} \lambda$, after sth incremental steps, corresponds to the summation of the load parameter in the previous step, $s-1$, and the incremental load factor. Then,

$$
{ }_{i}^{s} \lambda={ }^{s-1} \lambda+{ }_{i-1}^{s} \Delta \lambda+{ }_{i}^{s} \delta \lambda={ }^{s-1} \lambda+{ }^{s} \overline{\Delta \lambda}
$$

following the same idea of Equations (4.31) to (4.34), in which the term ${ }_{i-1}^{s} \Delta \lambda$ is null for $i=1$.

However, the LCM fails to trace nonlinear equilibrium paths through load limits points, because, in the vicinity of these points, the stiffness matrix becomes singular, and the iteration procedure diverges as ${ }_{i}^{s} \delta \boldsymbol{u} \rightarrow \infty$, i.e., the iterative updated displacement vector tends to be unbounded. This is a consequence of the load factor being the parameter control, and, wherefore, this divergence is inherent to this method (Yang \& Kuo, 1994).

\subsubsection{2}

\section{Arc-Length Method}

The Arc-Length Method (ALM), Crisfield (1981), is one of the most traditional nonlinear solution schemes, capable of handling with both load and displacement limit points. Mathematically, this method can be viewed as the trace of a single equilibrium curve in a space spanned by the nodal displacement variables $\boldsymbol{u}$ and the total load factor $\lambda$, cf. Figure 4.8. 


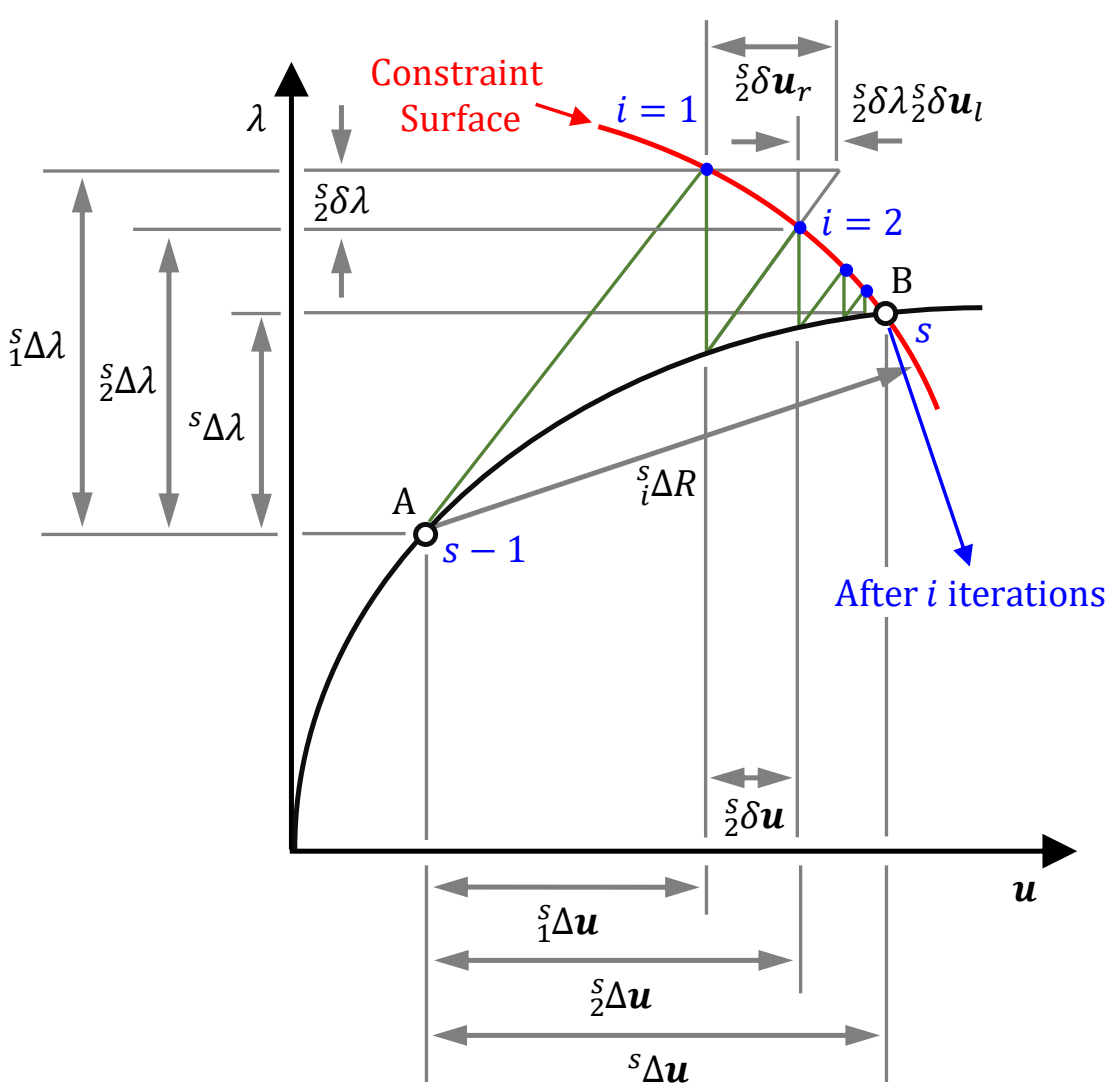

Figure 4.8: Arc-length method.

The general constraint equation for ALM, written with respect to the iterations, is

$$
{ }_{1}^{s} \delta \boldsymbol{u} \cdot{ }_{i}^{s} \delta \boldsymbol{u}+\beta_{1}^{2 s} \delta \lambda{ }_{i}^{s} \delta \lambda=\left({ }_{i}^{s} \Delta R\right)^{2},
$$

for ${ }_{i}^{s} \Delta R$ being the arc-length, i.e., is the radius of curvature, and $\beta$ is a nonnegative real parameter $\left(\beta \in \mathbb{R}_{+}\right)$. The square in $\beta$ is to make it compatible with classical literature, e.g., Forde \& Stiemer (1987). As summarized in Table 4.1, the value assigned for $\beta^{2}$ defines which version of arc-length constraints the solution path.

Table 4.1: Versions of arc-length method.

\begin{tabular}{c|c|c}
$\beta^{2}$ & Version & Equation \\
\hline 1 & Spherical & ${ }_{1}^{s} \delta \boldsymbol{u} \cdot{ }_{i}^{s} \delta \boldsymbol{u}+{ }_{1}^{s} \delta \lambda{ }_{i}^{s} \delta \lambda=\left({ }_{i}^{s} \Delta R\right)^{2}$ \\
\hline 0 & Cylindrical & ${ }_{1}^{s} \delta \boldsymbol{u} \cdot{ }_{i}^{s} \delta \boldsymbol{u}=\left({ }_{i}^{s} \Delta R\right)^{2}$ \\
\hline $\mathbb{R}_{+}^{*}-\{1\}$ & Elliptical & Equation $(4.46)$
\end{tabular}


By, simply inspection, it is possible the determine the restriction terms in Equation (4.39):

$$
\begin{gathered}
{ }_{i}^{s} \boldsymbol{a}={ }_{1}^{s} \delta \boldsymbol{u}={ }_{1}^{s} \delta \lambda_{1}^{s} \delta \boldsymbol{u}_{l} \\
{ }_{i}^{s} b=\beta_{1}^{2 s} \delta \lambda \\
{ }_{i}^{s} c=\left({ }_{i}^{s} \Delta R\right)^{2}= \begin{cases}\left({ }^{s} \overline{\Delta R}\right)^{2} & \text { for } i=1 \\
0 & \text { for } i \geqslant 2 .\end{cases}
\end{gathered}
$$

In the above set of equation, ${ }^{s} \overline{\Delta R}$ is the prescribed initial arc-length value, which does not have to be fixed for all incremental steps: it can assume different values in different increments according to the degree of nonlinearity involved in the equilibrium path, but it will be always defined in the first iteration of the sth step.

Since each incremental step $s$ begins at a previously converged step $s-1$, the unbalanced forces vector is null at the first iteration of step $s$. Therefore the iterative displacement residual portion ${ }_{i}^{s} \delta \boldsymbol{u}_{r}$ will also vanish according to Equation (4.42b). It follows from Equation (4.41) that

$$
{ }_{1}^{s} \delta \boldsymbol{u}={ }_{1}^{s} \delta \lambda{ }_{1}^{s} \delta \boldsymbol{u}_{l}
$$

and isolating the load factor in Equation (4.46) results in:

$$
{ }_{i}^{s} \delta \lambda= \begin{cases} \pm \frac{{ }^{s} \overline{\Delta R}}{\sqrt{{ }_{1}^{s} \delta \boldsymbol{u}_{l} \cdot{ }_{1}^{s} \delta \boldsymbol{u}_{l}+\beta^{2}}} & \text { if } i=1 \\ -\frac{{ }_{1}^{s} \delta \boldsymbol{u} \cdot{ }_{1}^{s} \delta \boldsymbol{u}_{r}}{{ }_{1}^{s} \delta \boldsymbol{u} \cdot{ }_{i}^{s} \delta \boldsymbol{u}_{l}+\beta_{1}^{2 s} \delta \lambda} & \text { otherwise. }\end{cases}
$$

It is worth to emphasize that the general constraint equation is quadratic in terms of ${ }_{i}^{s} \delta \lambda$. To solve it a set of rules must be adopted to treat real or complex roots and to define which of these roots will be used to evaluate the next point in the equilibrium solution.

This scheme has the ability to identify the change of direction in the load factor: the plus sign refers to loading and the minus sign means unloading. Nevertheless, one drawback of ALM has been the lack of information for determining the change in the sign of ${ }_{i}^{s} \delta \lambda$.

Another concerning fact is about the units of terms in Equation (4.49). The load factor ${ }_{i}^{s} \delta \lambda$ is a scalar, while displacements vectors ${ }_{i}^{s} \delta \boldsymbol{u}$ contain trans- 
lations and rotations, which are different in units and orders of magnitudes. Because of that, there is the possibility for the load parameter to be large so that the sign of it depends fully on the angle between ${ }_{1}^{s} \delta \boldsymbol{u}$ and ${ }_{i}^{s} \delta \boldsymbol{u}_{r}$. This is especially true in the vicinity of displacement limit points, where the gradient tends to be high. It follows that the sign of ${ }_{i}^{s} \delta \lambda$ may change improperly resulting in wrong iterative directions and ending in numerical divergence, i.e., the algorithm achieves the maximum number of allowed iterations without convergence. Leon et al. (2011) summarizes the difficulties find by researchers along the years and can be read for further discussion.

\subsubsection{3}

\section{Generalized Displacement Control Method}

The Generalized Displacement Control Method (GDCM) was proposed by Yang \& Shieh (1990) due to the limitations on the existing techniques. In its original formulation, based on ALM, the following constraint parameters were selected:

$$
\begin{aligned}
& { }_{i}^{s} \boldsymbol{a}={ }_{1}^{s} \delta \lambda^{s-1} \delta \boldsymbol{u}_{l} \\
& { }_{i}^{s} b=0 \\
& { }_{i}^{s} c= \begin{cases}\text { generalized displacement } & \text { for } i=1 \\
0 & \text { for } i \geqslant 2 .\end{cases}
\end{aligned}
$$

From equation (4.43), the load factor is

$$
{ }_{i}^{s} \delta \lambda=\frac{{ }_{i}^{s} c-{ }_{1}^{s} \delta \lambda\left({ }_{1}^{s-1} \delta \boldsymbol{u}_{l} \cdot{ }_{i}^{s} \delta \boldsymbol{u}_{r}\right)}{{ }_{1}^{s} \delta \lambda^{s-1} \delta \boldsymbol{u}_{l} \cdot{ }_{i}^{s} \delta \boldsymbol{u}_{l}},
$$

where the generalized displacement is prescribed on the first iteration of the incremental step (i.e., ${ }_{1}^{s} c=c$ ) and is unchanged at subsequent iterations. When $i=1,{ }_{i}^{s} \delta \boldsymbol{u}_{r}=\mathbf{0}$, and the load parameter can be written as:

$$
{ }_{i}^{s} \delta \lambda=\left\{\begin{array}{cl}
\frac{c}{{ }_{1}^{s} \delta \lambda^{s-1} \delta \boldsymbol{u}_{l} \cdot{ }_{1}^{s} \delta \boldsymbol{u}_{l}} & \text { if } i=1 \\
-\frac{{ }_{1}^{s-1} \delta \boldsymbol{u}_{l} \cdot{ }_{i}^{s} \delta \boldsymbol{u}_{r}}{{ }_{1} \delta \boldsymbol{u}_{l} \cdot{ }_{i}^{s} \delta \boldsymbol{u}_{l}} & \text { otherwise. }
\end{array}\right.
$$

Special treatment of Equation (4.52) is required for the first step because 
${ }_{1}^{0} \delta \boldsymbol{u}_{l}$ does not exist. Hence, the authors adopted ${ }_{1}^{0} \delta \boldsymbol{u}_{l}={ }_{1}^{1} \delta \boldsymbol{u}_{l}$, which results in

$$
c=(\overline{\delta \lambda})^{2}\left({ }_{1}^{1} \delta \boldsymbol{u}_{l} \cdot{ }_{1}^{1} \delta \boldsymbol{u}_{l}\right)
$$

for ${ }_{1}^{1} \delta \lambda=\overline{\delta \lambda}$ being the prescribed initial control factor. Ergo, the expression for ${ }_{1}^{s} \delta \lambda$ becomes:

$$
{ }_{1}^{s} \delta \lambda= \pm \overline{\delta \lambda}\left(\left|\frac{{ }_{1}^{1} \delta \boldsymbol{u}_{l} \cdot{ }_{1}^{1} \delta \boldsymbol{u}_{l}}{{ }^{s-1} \delta \boldsymbol{u}_{l} \cdot{ }_{1}^{s} \delta \boldsymbol{u}_{l}}\right|\right)^{\frac{1}{2}}
$$

The ratio in the parentheses is the modulus of a quantity known as generalized stiffness parameter,

$$
G S P=\frac{{ }_{1}^{1} \delta \boldsymbol{u}_{l} \cdot{ }_{1}^{1} \delta \boldsymbol{u}_{l}}{{ }_{1}^{s-1} \delta \boldsymbol{u}_{l} \cdot{ }_{1}^{s} \delta \boldsymbol{u}_{l}}
$$

which plays an important role in the change of load direction. GSP is positive for stiffening systems and negative for those presenting softening behavior. Its numerator represents the norm of iterative displacement at the first step, while the denominator is, approximately, the norm of iterative displacement at the current step. As consequence, GSP symbolizes the stiffness of a structure (in the current step referred to the first step). Another important feature is that the sign of GSP is positive, except immediately after load limit points, where $G S P=0$. The reason for that is because it depends purely of the scalar product ${ }_{1}^{s-1} \delta \boldsymbol{u}_{l} \cdot{ }_{1}^{s} \delta \boldsymbol{u}_{l}$.

Considering the characteristics of $G S P,{ }_{1}^{s} \delta \lambda$ can be written, perhaps in a more understandable and direct form, as

$$
{ }_{1}^{s} \delta \lambda=\operatorname{sign}(G S P) \overline{\delta \lambda}(|G S P|)^{\frac{1}{2}},
$$

where "sign" is the GSP sign.

The capability of changing the load sign in the correct points (only at load limit points) makes this method very robust and one of the best options to capture complex nonlinear behaviors. In addition, the load increment can be adjusted according to the nonlinearities of the structure and numerical stability is always achieved in regions near the critical points, i.e., both load factor and displacements are bounded. 


\section{6}

\section{Flowchart}

A flowchart for the optimization process is illustrated in Figure 4.9. It can be divided into three parts. The first one concerns to the mesh generation in which the geometry (length, height and thickness), the number of elements and the symmetry conditions (if applied) are defined. The mesh itself can be generated by commercial software, although it has been used the PolyMesher function (Talischi et al., 2012a) for that. This function returns nodal coordinates, applied loads and supports and a cell of element connectivities. Material properties such as Poisson's coefficient and Young's modulus also must be specified.

The second part is about the nonlinear solution. The original PolyTop code (Talischi et al., 2012b), regarded only for linear analysis, was modified to solve geometrical nonlinear problems for the methods of LCM or GDCM. The algorithm, implemented in Matlab ${ }^{\circledR}$ (MATLAB, 2017), also includes shape functions for Q4 and Q8 elements. In this part, one of these schemes is set alongside with the maximum number of steps, the maximum number of iterations to be taken by each step, the convergence tolerance $(C O N V)$ and the initial load factor $\bar{s} \Delta \lambda$. Those parameters are summarized in Table 4.2.

The last portion considers the optimization parameters. The user defines the filter radius, the volume fraction, $\beta_{1}$ or $\beta_{2}$ value, the design variable tolerance TOL, the value of $p$ used in the continuation scheme and the optimizer, OC or MMA, and its input parameters. The maximum number of iterations allowed Iter $_{\max }$ may remain fixed during the optimization process, but it also may vary as $p$ is increased (see Chapter 5).

Table 4.2: Input data for the Newton-Raphson procedure.

\begin{tabular}{c|c} 
Parameter & Value \\
\hline Max. steps allowed & 20 \\
\hline Max. iterations allowed & 200 \\
\hline Reference load $(\overline{\boldsymbol{l}})$ & $\boldsymbol{q}^{\dagger}$ \\
\hline Initial load factor $\left(\overline{s^{\Delta} \lambda}\right)$ & $\begin{array}{c}\text { Cantilever beam: } 0.05\|\boldsymbol{q}\| \\
\text { Clamped beam: } 0.5\|\boldsymbol{q}\|\end{array}$ \\
\hline Convergence tolerance $(C O N V)$ & $\left\|{ }_{i}^{s} \delta \boldsymbol{u}\right\|=10^{-6}$
\end{tabular}

${ }^{\dagger} \boldsymbol{q}$ is the applied load vector 


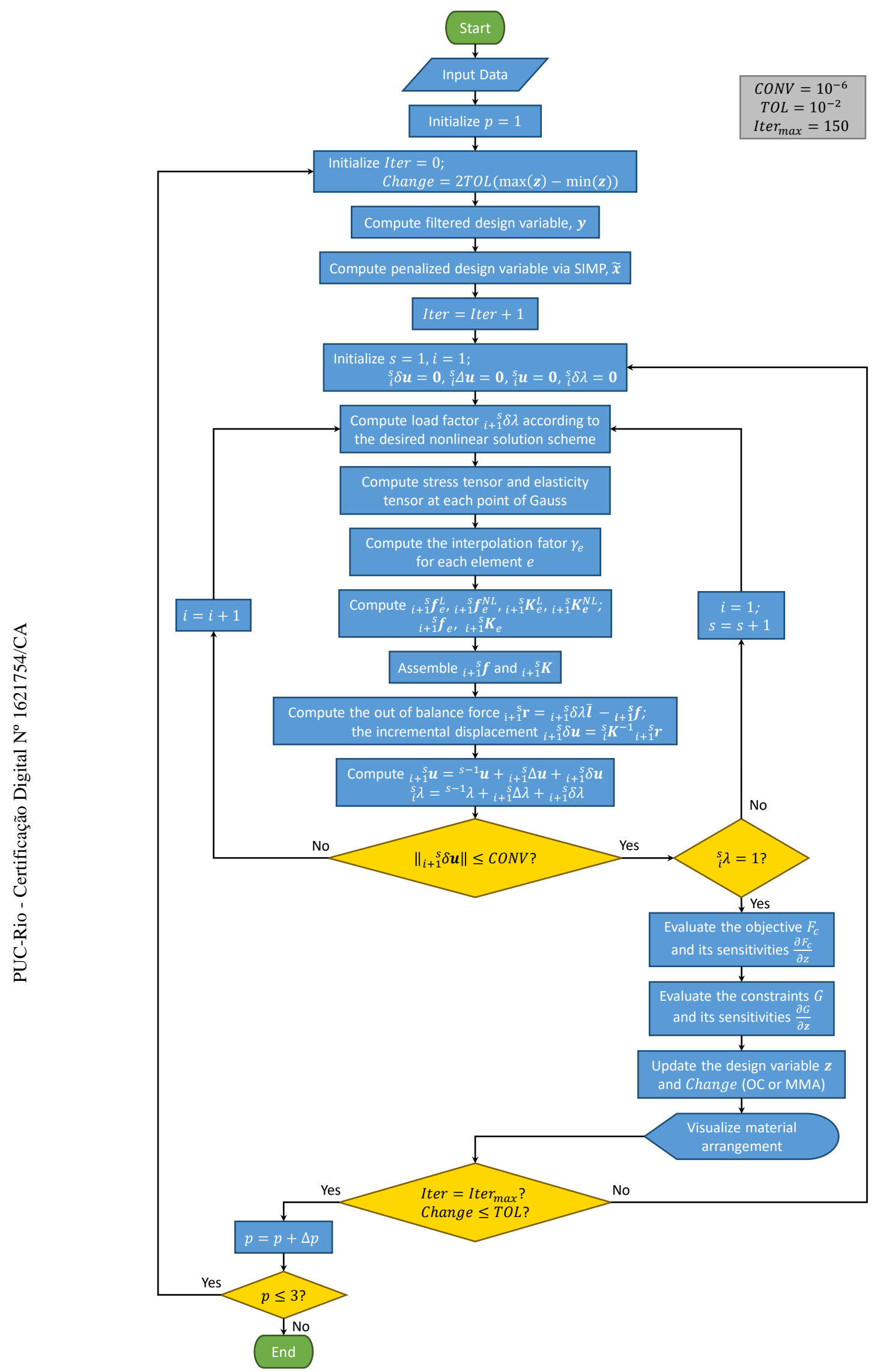

Figure 4.9: Flowchart for geometric nonlinear topology optimization. 


\section{5 \\ Results}

\section{1}

\section{Introduction}

This chapter is dedicated to illustrate examples using the interpolation scheme discussed in Section 4.3. Two structures are investigated: the cantilever and the clamped beams. All results are conducted for a plane strain state ${ }^{1}$, considering, as the objective function, the the "end-compliance" at the load application point. Different load levels are considered for the same structure and the nonlinear state equation is solved by the load control method at each step of the optimization. The implemented algorithm always ensure that the external force reaches its maximum value during the optimization process. The MMA solver (Svanberg, 1987) is applied to solve the topology optimization problem.

\section{2}

\section{Cantilever Beam}

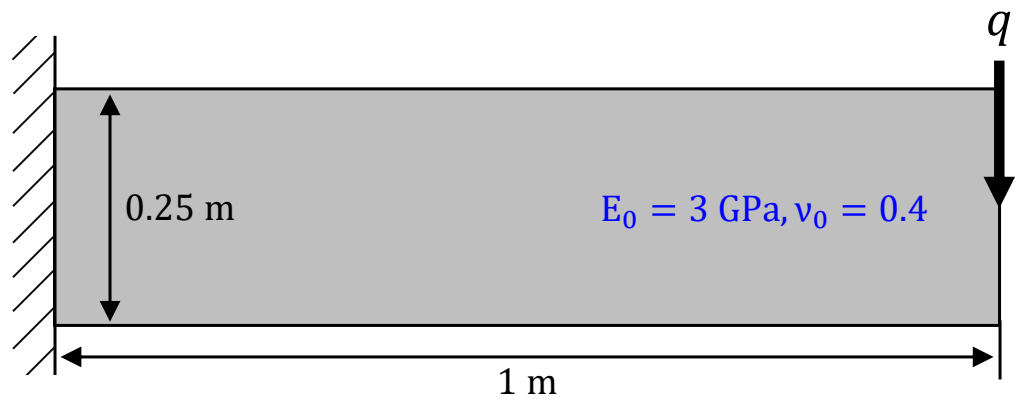

Figure 5.1: Cantilever beam domain.

The first structure is the cantilever beam (Buhl et al., 2000), shown in Figure 5.1. The beam is $1 \mathrm{~m}$ long $(L), 0.25 \mathrm{~m}$ height $(H)$ and $0.1 \mathrm{~m}$ depth $(d)$, the Young's modulus $E_{0}$ is $3 \mathrm{GPa}$ and the Poisson's coefficient $\nu_{0}$ is 0.4 . This model is fixed on the left edge and loaded downwards at the midpoint of the

\footnotetext{
${ }^{1}$ Beams are often studied under the plane stress state assumption, but most of the works in literature treats these examples as plane strain. So, the plane strain state was adopted in all analysis in order to perform a fair comparison to the literature.
} 
right edge by a force of magnitude $q$. The design domain is discretized by 120 x 30 Q4 elements and the volume fraction is $V_{\text {frac }}=0.5$.

The penalization exponent starts at $p=1$ and is increased by $\Delta p=0.05$ until it reaches a maximum value of 3 following the continuation method. The same procedure adopted by Wang et al. (2014) is considered here: until $p<2$ the optimization iterations are restricted to 2 ; from $2 \leqslant p<3$, a maximum of 5 iterations are allowed. When $p=3$, the optimization algorithm stops only if it converges or reaches the maximum iterations allowed Iter $_{\max }=150$. The filter radius is $r_{\min }=H / 8$.

The SIMP method is employed until the penalization factor achieves its maximum value, i.e., $p=3$, then the tanh projection function is combined with it, as defined in Equations (3.8) and (3.9). In this manner, the material interpolation, given by the combination of SIMP and tanh function, does not influence the convergence of the nonlinear solution method. And more, it plays a crucial role to reduce the compliance value since it diminish the grays on the boundaries of the optimized design. Not combining (nonlinear) projection functions to SIMP will result in similar structures, but the large presence of intermediate densities in their layouts tends to make the final topology stiffer than those presented in here.

Table 5.1 compares the optimal topologies for the linear and nonlinear cases without employing the interpolation scheme. For small magnitudes of loads, such as $6 \mathrm{kN}$ and $12 \mathrm{kN}$, one can observe the similiarities between the small and large deformation theories. For instance, the difference in the compliance $F_{c}$ is very small and can be considered irrelevant. The higher the force, the higher is the difference between these formulations. This is perceptible due to the loss of symmetry in higher loads: as the force increases, the responses in the displacements also increase and the sensitivity values also change.

When the interpolation scheme is employed, the optimal design for the loads of 240 and $300 \mathrm{kN}$ - which the solver was not capable to determine assuming nonlinear behavior due to numerical instabilities - now could be achieved as printed out in Figure 5.2. Loads of 12, 60, 96 and $144 \mathrm{kN}$ presents very similar topologies to those obtained without evoking the interpolation scheme (Table 5.1), demonstrating that this technique does not influence on the behavior of structural elements.

The element's behavior for a force of $300 \mathrm{kN}$ is exhibited in Figure 5.3. As expected, the structural elements are characterized by large displacements and the low-stiffness ones by small displacements. 
Table 5.1: Cantilever beam optimal topologies and compliance values for several load levels considering linear and nonlinear analyses.

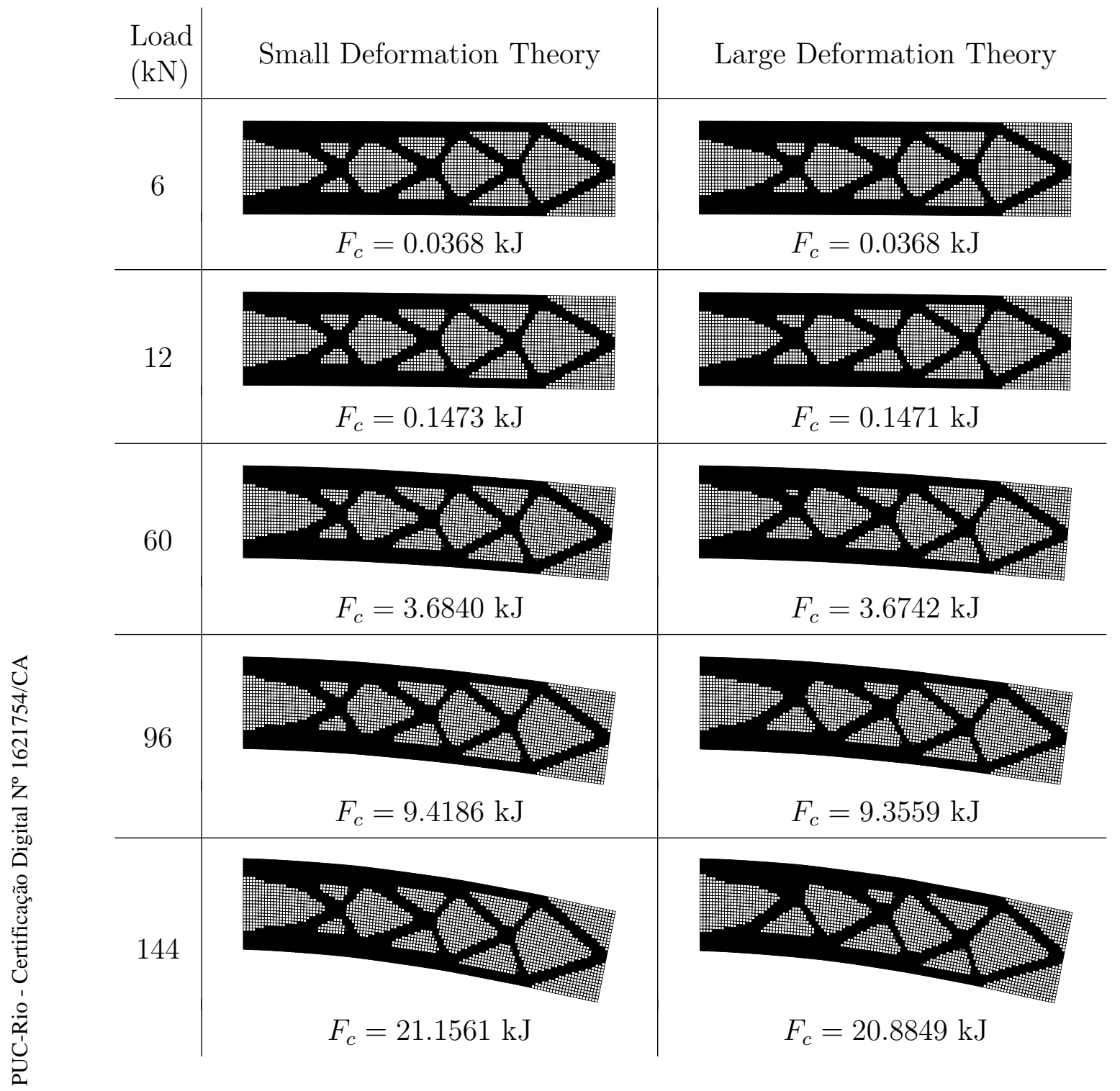




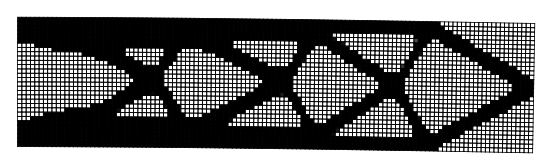

$5.2(\mathrm{a}): 12 \mathrm{kN}, F_{c}=0.1469 \mathrm{~kJ}$.

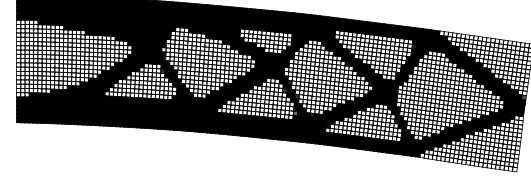

$5.2(\mathrm{c}): 96 \mathrm{kN}, F_{c}=9.3525 \mathrm{~kJ}$.

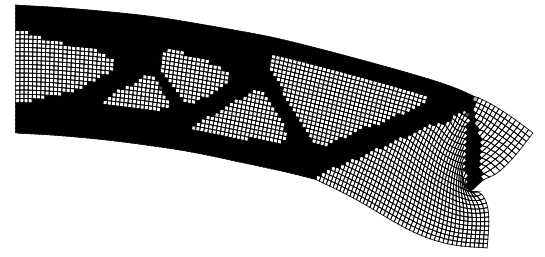

$5.2(\mathrm{e}): 240 \mathrm{kN}, F_{c}=56.3350 \mathrm{~kJ}$.

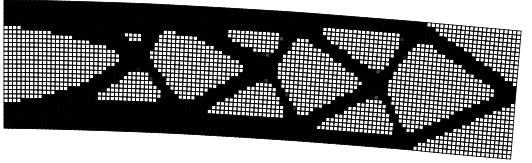

$5.2(\mathrm{~b}): 60 \mathrm{kN}, F_{c}=3.6702 \mathrm{~kJ}$.

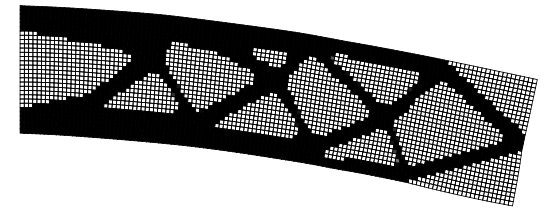

$5.2(\mathrm{~d}): 144 \mathrm{kN}, F_{c}=20.8792 \mathrm{~kJ}$.

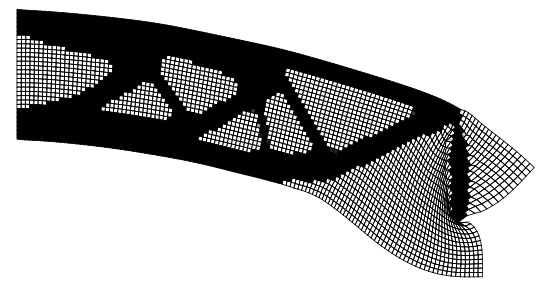

$5.2(\mathrm{f}): 300 \mathrm{kN}, F_{c}=84.8276 \mathrm{~kJ}$.

Figure 5.2: Optimal topologies and corresponded compliance values for the indicated loads obtained using the interpolation scheme.

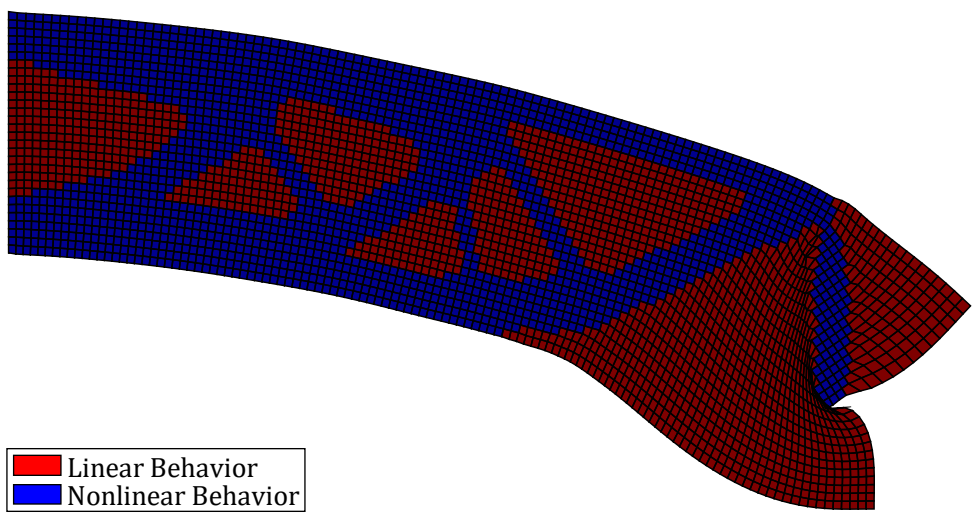

Figure 5.3: Element's behavior at the end of the optimization process for an applied force of $300 \mathrm{kN}$.

\subsection{1}

\section{Comparison with Literature}

In this section, the values of the "end-compliance" and the optimal topologies are compared to the results in the recent literature concerning the optimization of geometric nonlinear structures. For this, two references are considered: Lahuerta et al. (2013) and Wang et al. (2014).

The comparison is taken for the appiled forces of 60,144 and $300 \mathrm{kN}$, cf. Table 5.2. In each load level, there are similarities on both topologies and objective values. 
Table 5.2: Comparison of optimized topologies for the cantilever beam for three different loads.

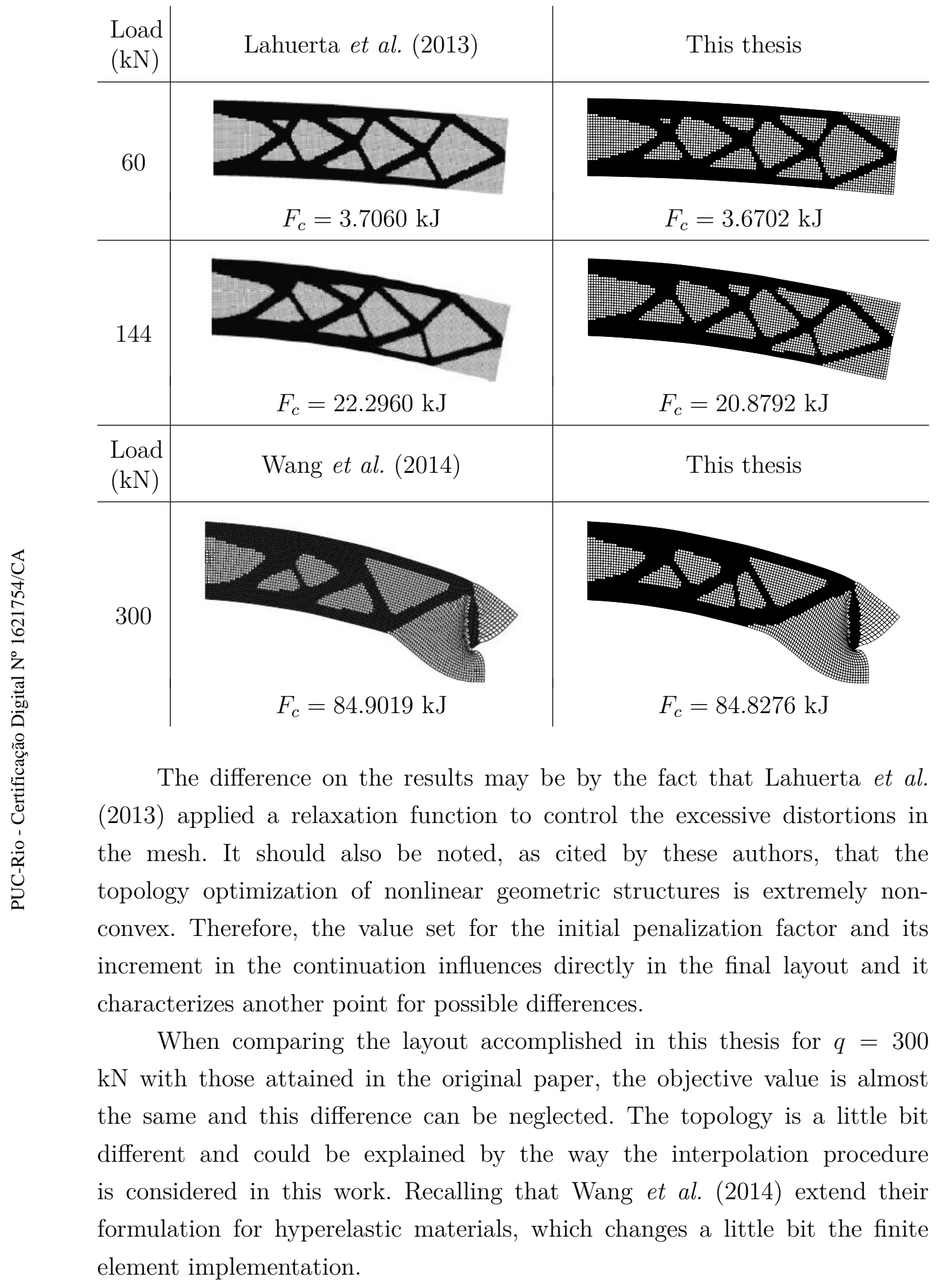




\section{3}

\section{Clamped Beam}

The second example is the clamped beam (Buhl et al., 2000). This beam is encastred and the force of magnitude $q$ is applied at midpoint on the upper edge, cf. Figure 5.4. The geometry is $L \times H \times d=3 \times 1 \times 0.1 \mathrm{~m}^{3}$, the Young's modulus $E_{0}$ and the Poisson's coefficient $\nu_{0}$ are, respectively, $3 \mathrm{GPa}$ and 0.4 . The design domain is discretized by $120 \times 40$ Q4 elements and the final volume $V_{f}$ is set be $10 \%$ of the initial volume $V_{0}$.

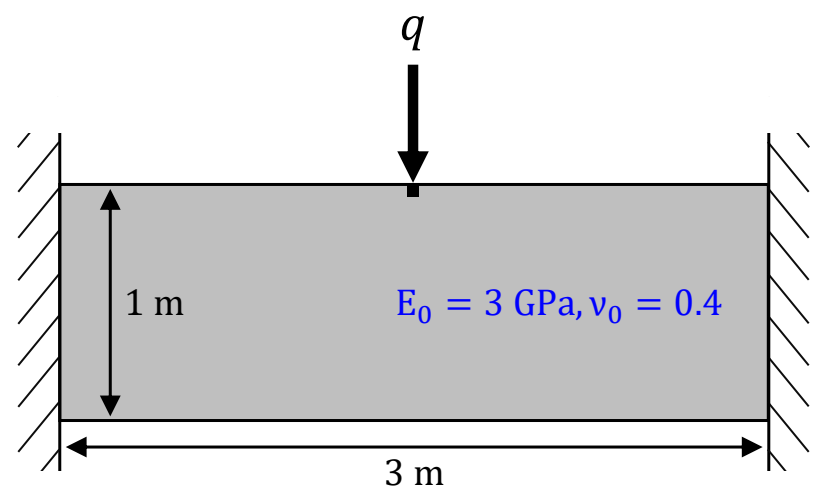

Figure 5.4: Clamped beam domain.

This structure exhibits large distortions on elements around the point of load application, because the volume fraction is small $\left(V_{\text {frac }}=0.1\right)$. To relax the mesh distortion in the vicinity of the loading point, the force is uniformly distributed over the nodes of four adjacent elements, as indicated by the black square box below the load point. This is a common practice in the literature, e.g., Luo et al. (2015). For the cantilever example, this load distribution is not necessary, because the volume fraction will not cause immediately instability in low-density regions, as this structure allows a higher amount of volume in the optimal solution.

The continuation strategy applied for the cantilever beam is the same adopted in this example, except by the increment $\Delta p$, which is set to 0.1 . It is possible to start the penalization factor from a higher value, e.g., $p=2$, as done by Luo \& Tong (2016) and Wallin et al. (2018). This will prevent the algorithm to former the topology of linear analysis initially. However, to apply the interpolation method and allow it to penalize the low-density elements, the continuation scheme must starts at $p=1$.

A different strategy is considered to reduce the grayish tones. The number of iterations taken for the load of $10 \mathrm{kN}$ is very small and does not justify the use of projection functions in the same manner as described for the cantilever beam. So, at the end of optimization, the effect of the filter is eliminated. In 
short, the optimization procedure is conducted using the SIMP method and a filter of radius $r_{\min }=H / 20$. When $p=3$ the filter influence is removed and the algorithm runs until the solution converges.

The topologies for the clamped beam are displayed in Table 5.3 for analysis considering small deformations and the interpolation scheme. The similarities in the optimal layouts for the load of $10 \mathrm{kN}$, that has a linear behavior, and the differences for $230 \mathrm{kN}$, characterized by large displacements, are notables.

Table 5.3: Clamped beam optimal topologies and compliance values for two load levels considering linear analysis and the interpolation scheme.

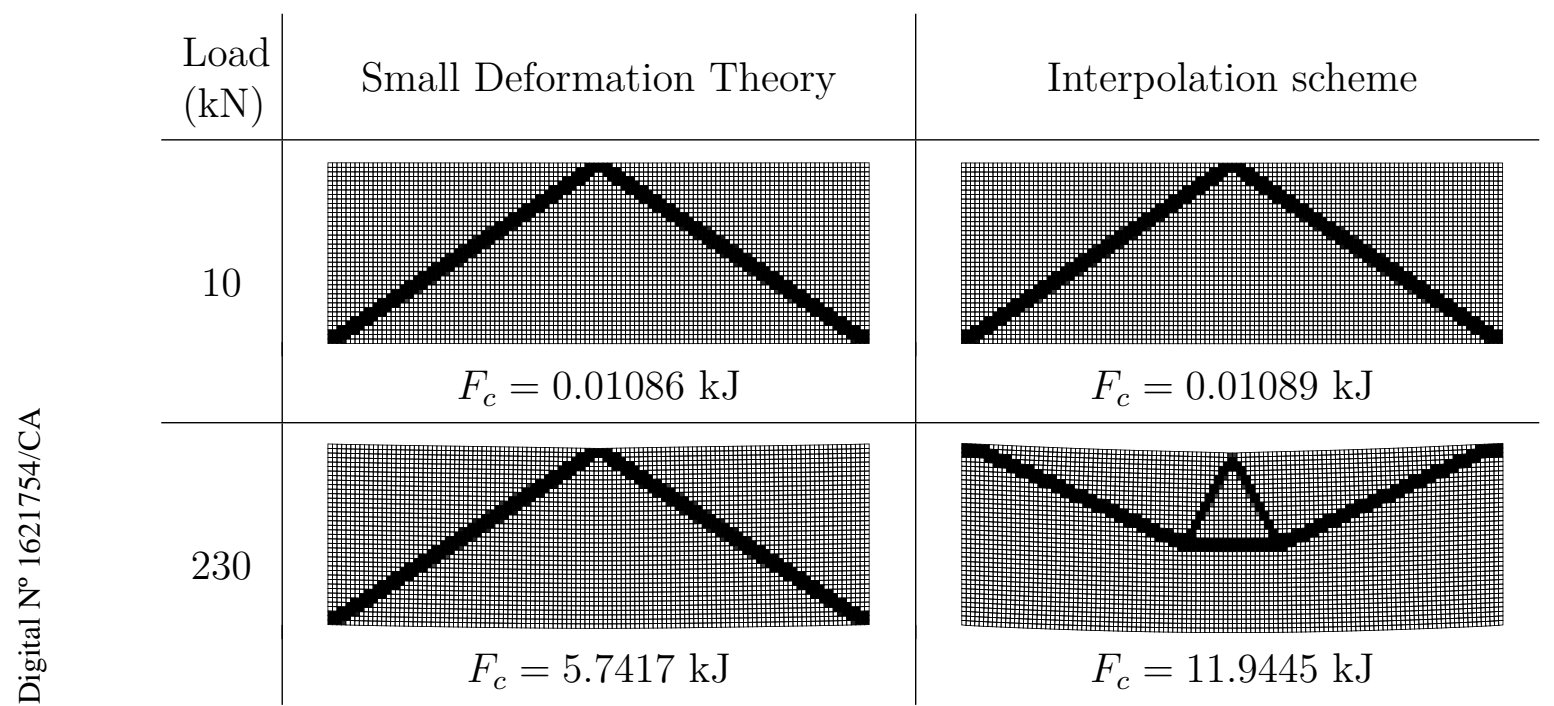

It is worth to mention that all these responses were achieved through simulations and the objective values have precision of four or five decimal places. So for these reasons, in the linear analysis, for $230 \mathrm{kN}$ the compliance indicated in Table 5.3 presents a small difference from the expected value: $23^{2} \cdot 0.01086=5.74494 \mathrm{~kJ}$.

\subsection{1}

\section{Comparison with Literature}

The values of the objective function and the optimal topologies are compared to the results from Lahuerta et al. (2013) and Wang et al. (2014) for the load of $230 \mathrm{kN}$. As can be seen in Table 5.4, the topologies and the compliance $F_{c}$ are very similar.

The evaluated objective is greater than the one indicated in the work of Wang et al. (2014). This is explained by the use of a different procedure to reduce the intermediate stiffness in the final solution. By simple inspection, it is possible to say that eliminating the filtering influence, as done here, leaves approximately twice as many elements with intermediate densities as compared 
to the original paper, where the tanh projection function was considered. Another distinction is related to the force distribution, which seems not to be adopted by those authors, yet it leads to a different material arrangement in the vicinity of the load application point.

In relation to the paper of Lahuerta et al. (2013), the difference, not only in the objective value but also in the optimal layout, can be explained by the mesh discretization. In their analysis, the authors considered 60,000 Q4 elements. The investigated interpolation approach has demonstrated to be sensitive to mesh-refinement and simulations employing refined meshes like that may recover the undesirable numerical instabilities.

Table 5.4: Comparison of optimized topologies for the clamped beam for a load of $230 \mathrm{kN}$.

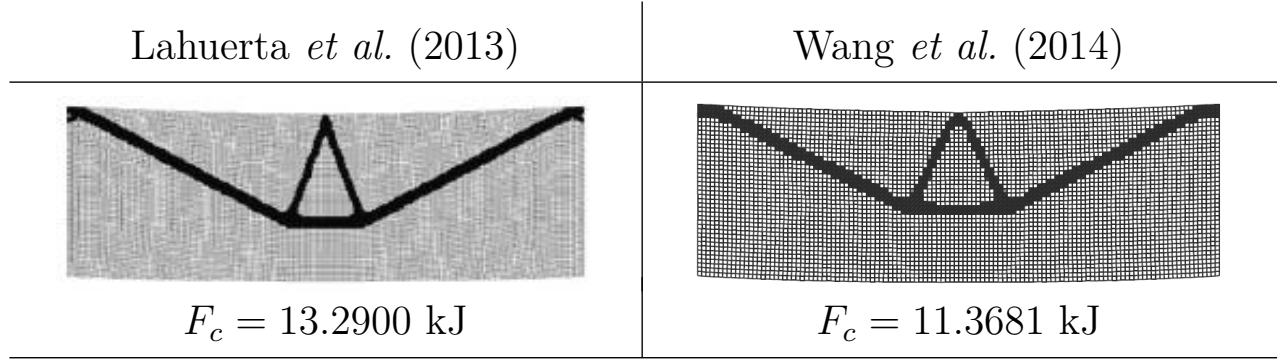

This thesis

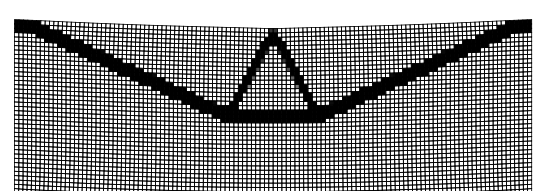

$F_{c}=11.9445 \mathrm{~kJ}$ 


\section{6 \\ Conclusions}

In this thesis, an interpolation scheme (Section 4.3) was considered. The idea is to alleviate numerical instabilities in low-density (void) regions during the topology optimization of structures undergoing large displacements. Inspired by the fact that the stored elastic energy density function can be interpolated between the small and the large deformation theories, this scheme was implemented in such a way that it can be seen as a simple modification on the element stiffness matrix and internal force vector.

The interpolation method was validated throughout two common examples in the study of topology optimization regarding geometric nonlinearities. By means of cantilever and clamped beams this approach alleviated the numerical difficulties in low-stiffness regions within the design domain and the convergence to a final layout was possible.

Although the penalty function (SIMP model) penalizes intermediate densities, some gray regions occur due to the filter operation - the boundaries of the optimal topology still characterized by shades of gray. These intermediate densities are reduced by composing the tanh projection function with SIMP, which modifies the material interpolation equation. Nevertheless, it had been observed, in the earlier steps of continuation, that this composition may still result in numerical instabilities of low-density areas. This was a direct consequence from changing the material interpolation function, which also modified the element densities and, thus, the material distribution. For this reason, the tanh function was only applied at the last step of the continuation, where the density of elements was no longer susceptible to large variations and the material arrangement was closer to the optimal solution.

As could be seen in the clamped beam case, turning off the filter in the final continuation was also an effective way of reducing the grays on the topology boundaries. This is a more generic technique, without the restrictions and difficulties aforementioned for the tanh projection function.

Regarding the nonlinear solution method, should be mentioned that the LCM can be easily replaced by the GDCM in the implemented algorithm. This allows capturing topologies whose applied loads are in the unstable part 
of equilibrium path or in structures where buckling is a relevant issue. The convergence criterium for these methods involves the norm of incremental displacement, which avoids low-density elements to interfere in the convergence. For example, as explained by Lahuerta (2012), when the convergence criterium is based on residual forces, the internal force vector can be modified by large displacements of void elements and may result in a fake convergence, impacting in the optimization procedure.

\section{1}

\section{Suggestions for Future Works}

Ending research with some questions in mind is not ideal, but principally due to the complexity involved in these problems and the amount of time expended in each analysis, there are some points that can be explored in further studies. To the best of author's knowledge, up to the date of this thesis' conclusion, the works in the literature concerning nonlinear topology optimization did not came up with a definitive solution to control the instabilities in void regions. Mainly, because the proposed methods either depend on a series of parameters or they are not robust in the sense that small changes in the initial conditions of the problem may result in non-convergence of the optimization process.

The work on this thesis can be extended to investigate compliant mechanisms, which is a tendency in the recent literature about topology optimization of nonlinear problems. Other objective functions can be analyzed, as the strain energy or the complementary work. The research in the field of nonlinear topology optimization can be extended to consider polygonal meshes or to include tridimensional studies or even to evaluate the plasticity effect. Beyond that, stress constraints can be added to the proposed optimization problem.

The MMA solver demonstrated an oscillatory behavior, and it should be considered a more in deep look on it to better understand and manipulate all their input variables and controlling parameters, allowing to improve the code efficiency.

One way of improving the robustness of the nonlinear topology optimization is using hyperelastic material models, as the neo-Hookean. The recent reference literature has evoked these models, which are known to better represent the physics of a body under large compressions (Lahuerta, 2012). Despite the pure application of these models tends not to solve the convergence issues completely, combining them with specific techniques - as the interpolation debated here - allows the algorithm to treat more complex problems where the deformations are not properly described by the SVK model.

The interpolation scheme discussed in this thesis has alleviated numerical 
instabilities in low-density elements. Nonetheless, the convergence of the geometric nonlinear topology optimization process is extremely mesh-dependent. Refining the mesh will reduce the elements average size and their degrees of freedom will be relatively larger (to themselves), possibly resulting in large distortions of voids and recovering the undesired numerical instabilities. This enforces the need for exploring different approaches in the nonlinear topology optimization problems. Instead of penalizing the displacements or the density of elements, perhaps a technique that considers deformations (Luo et al., 2015) is a way out: as the strains are relative to the body configuration, it can be modified at every step of the optimization accordingly to the observed instabilities. This mesh-dependence can be listed as one of the reasons for this theme still drawing attention from researchers: topology optimization of geometrically nonlinear structures is still an open problem. 


\section{Bibliography}

APDL. 2017. ANSYS ${ }^{\circledR}$, Help System, Release 18.2. ANSYS, Inc. ANSYS ${ }^{\circledR}$ Mechanical APDL Product Launcher.

Ball, J.M. 1976. Convexity conditions and existence theorems in nonlinear elasticity. Archive for Rational Mechanics and Analysis, 63(4), 337-403.

Bathe, K.J. 2014. Finite Element Procedures. 2nd edn. Upper Saddle River, NJ: Prentice Hall.

Bendsøe, M.P. 1989. Optimal shape design as a material distribution problem. Structural and Multidisciplinary Optimization, 1(4), 193-202.

Bendsøe, M.P., \& Kikuchi, N. 1988. Generating optimal topologies in structural design using a homogenization method. Computer Methods in Applied Mechanics and Engineering, 71(2), 197-224.

Bendsøe, M.P., \& Sigmund, O. 1999. Material interpolation schemes in topology optimization. Archive of Applied Mechanics, 69(9), 635-654.

Bendsøe, M.P., \& Sigmund, O. 2003. Topology Optimization: Theory, Methods and Applications. 2nd edn. Berlin, Germany: Springer-Verlag Berlin Heidelberg.

Bendsøe, M.P., \& Sigmund, O. 2007. Topology Optimization. Chap. 6, pages 161-194 of: Jasbir, S.A. (ed), Optimization of Structures and Mechanical Systems. Toh Tuck, Singapure: World Scientific Publishing.

Bonet, J., \& Wood, R.D. 2008. Nonlinear Continuum Mechanics for Finite Element Analysis. 2nd edn. New York, NY: Cambridge University Press.

Bruns, T.E., \& Tortorelli, D.A. 1998. Topology optimization of geometrically nonlinear structures and compliant mechanisms. Pages 1874-1882 of: Proceedings of the 7th AIAA/USAF/NASA/ISSMO Symposium on Multidisciplinary Analysis and Optimization.

Bruns, T.E., \& Tortorelli, D.A. 2001. Topology optimization of non-linear elastic structures and compliant mechanisms. Computer Methods in Applied Mechanics and Engineering, 190(26-27), 3443-3459. 
Bruns, T.E., \& Tortorelli, D.A. 2003. An element removal and reintroduction strategy for the topology optimization of structures and compliant mechanisms. International Journal for Numerical Methods in Engineering, 57(10), 1413-1430.

Buhl, T., Pedersen, C.B.W., \& Sigmund, O. 2000. Stiffness design of geometrically nonlinear structures using topology optimization. Structural and Multidisciplinary Optimization, 19(2), 93-104.

Chen, Q., Zhang, X., \& Zhu, B. 2018. A 213-line topology optimization code for geometrically nonlinear structures. Structural and Multidisciplinary Optimization, 1-17.

Chi, H., da Veiga, L.B., \& Paulino, G.H. 2017. Some basic formulations of the virtual element method (VEM) for finite deformations. Computer Methods in Applied Mechanics and Engineering, 318, 148-192.

Crisfield, M.A. 1981. A fast incremental/iterative solution procedure that handles "snap-through". Pages 55-62 of: Computational Methods in Nonlinear Structural and Solid Mechanics. Elsevier.

da Senhora, F.V. 2017. Topology Optimization with Stress Constraints: an Aggregation-Free Approach. Master Dissertation (in Portuguese), Department of Mechanical Engineering, Pontifical Catholic University of Rio de Janeiro, Rio de Janeiro, Brazil.

Diaz, A., \& Sigmund, O. 1995. Checkerboard patterns in layout optimization. Structural optimization, 10(1), 40-45.

Forde, B.W.R., \& Stiemer, S.F. 1987. Improved arc length orthogonality methods for nonlinear finite element analysis. Computers \& structures, $27(5), 625-630$.

Gea, H.C., \& Luo, J. 2001. Topology optimization of structures with geometrical nonlinearities. Computers \& Structures, 79(20), 1977-1985.

Gomes, F.A.M., \& Senne, T.A. 2014. An algorithm for the topology optimization of geometrically nonlinear structures. International Journal for Numerical Methods in Engineering, 99(6), 391-409.

Guest, J.K., Prévost, J.H., \& Belytschko, T. 2004. Achieving minimum length scale in topology optimization using nodal design variables and projection functions. International journal for numerical methods in engineering, 61(2), 238-254. 
Haber, R.B., Jog, C. S, \& Bendsøe, M.P. 1996. A new approach to variabletopology shape design using a constraint on perimeter. Structural optimization, 11(1-2), 1-12.

Holtzer, B.P.F. 2017. Topology Optimization of Geometrically Nonlinear Structures. Master Dissertation, Department of Precision and Microsystems Engineering, Delft University of Technology, Delft, Netherlands.

Huang, X., \& Xie, Y.M. 2008. Topology optimization of nonlinear structures under displacement loading. Engineering Structures, 30(7), 2057-2068.

Jog, C. 1996. Distributed-parameter optimization and topology design for non-linear thermoelasticity. Computer Methods in Applied Mechanics and Engineering, 132(1-2), 117-134.

Jog, C.S., \& Haber, R.B. 1996. Stability of finite element models for distributed-parameter optimization and topology design. Computer methods in applied mechanics and engineering, 130(3-4), 203-226.

Jung, D., \& Gea, H.C. 2004. Topology optimization of nonlinear structures. Finite Elements in Analysis and Design, 40(11), 1417-1427.

Kawamoto, A. 2009. Stabilization of geometrically nonlinear topology optimization by the Levenberg-Marquardt method. Structural and Multidisciplinary Optimization, 37(4), 429-433.

Kemmler, R., Lipka, A., \& Ramm, E. 2005. Large deformations and stability in topology optimization. Structural and Multidisciplinary Optimization, 30(6), 459-476.

Kim, N.H. 2015. Introduction to Nonlinear Finite Element Analysis. New York, NY: Springer.

Lahuerta, R.D. 2012. Design of Structures Considering the Effect of Geometric Nonlinearity Using the Topological Optimization Method. Master Dissertation (in Portuguese), Department of Mechatronics Engineering and Mechanical Systems, University of São Paulo, São Paulo, Brazil.

Lahuerta, R.D., Simões, E.T., Campello, E.M.B, Pimenta, P.M., \& Silva, E.C.N. 2013. Towards the stabilization of the low density elements in topology optimization with large deformation. Computational Mechanics, 52(4), 779-797. 
Lee, H.A., \& Park, G.J. 2012. Topology optimization for structures with nonlinear behavior using the equivalent static loads method. Journal of Mechanical Design, 134(3), 031004.

Leitão, A.X., \& Pereira, A. 2018. Energy interpolation scheme for topology optimization of nonlinear elastic structures. In: Proceedings of the $X$ Congresso Nacional de Engenharia Mecânica. Salvador, BA, Brazil.

Leitão, A.X., \& Pereira, A. 2019. Topology Optimization of Geometrically Nonlinear Structures Considering an Energy Interpolation Scheme. In: Proceedings of the 25th Brazilian Congress of Mechanical Engineering (in press). Uberlândia, MG, Brazil.

Leon, S.E., Paulino, G.H., Pereira, A., Menezes, I.F.M., \& Lages, E.N. 2011. A unified library of nonlinear solution schemes. Applied Mechanics Reviews, 64(4), 040803.

Long, Y.Q., Song, C., \& Long, Z.F. 2009. Advanced Finite Element Method in Structural Engineering. Beijing, China: Tsinghua University Press and Springer-Verlag Berlin Heidelberg.

Luo, Q., \& Tong, L. 2016. An algorithm for eradicating the effects of void elements on structural topology optimization for nonlinear compliance. Structural and Multidisciplinary Optimization, 53(4), 695-714.

Luo, Y., Wang, M.Y., \& Kang, Z. 2015. Topology optimization of geometrically nonlinear structures based on an additive hyperelasticity technique. Computer methods in applied mechanics and engineering, 286, 422-441.

Mase, G.T., \& Mase, G.E. 1999. Continuum Mechanics for Engineers. 2nd edn. Boca Raton, FL: CRC Press.

MATLAB. 2017. version 9.3.0,681905 (R2017b). Natick, MA: The MathWorks, Inc.

Pajot, J.M. 2006. Topology Optimization of Geometrically Nonlinear Structures Including Thermo-mechanical Coupling. Ph.D. Thesis, Department of Aerospace Engineering Science, University of Colorado at Boulder, Boulder, USA.

Parente, E.J., \& Vaz, L.E. 2001. Improvement of semi-analytical design sensitivities of non-linear structures using equilibrium relations. International Journal for Numerical Methods in Engineering, 50(9), 2127-2142. 
Petersson, J., \& Sigmund, O. 1998. Slope constrained topology optimization. International Journal for Numerical Methods in Engineering, 41(8), 1417-1434.

Ramiere, I., Angot, P., \& Belliard, M. 2007. A fictitious domain approach with spread interface for elliptic problems with general boundary conditions. Computer Methods in Applied Mechanics and Engineering, 196(4-6), 766-781.

Reddy, J. 2008. An introduction to Continuum Mechanics with Applications. New York, NY: Cambridge University Press.

Sigmund, O., \& Petersson, J. 1998. Numerical instabilities in topology optimization: a survey on procedures dealing with checkerboards, meshdependencies and local minima. Structural optimization, 16(1), 68-75.

Svanberg, K. 1987. The method of moving asymptotes-a new method for structural optimization. International journal for numerical methods in engineering, 24(2), 359-373.

Talischi, C., Paulino, G.H., \& Le, C.H. 2009. Honeycomb Wachspress finite elements for structural topology optimization. Structural and Multidisciplinary Optimization, 569-583.

Talischi, C., Paulino, G.H., Pereira, A., \& Menezes, I.F.M. 2012a. PolyMesher: a general-purpose mesh generator for polygonal elements written in Matlab. Structural and Multidisciplinary Optimization, 45(3), 309-328.

Talischi, C., Paulino, G.H., Pereira, A., \& Menezes, I.F.M. 2012b. PolyTop: a Matlab implementation of a general topology optimization framework using unstructured polygonal finite element meshes. Structural and Multidisciplinary Optimization, 45(3), 329-357.

Talischi, C., Pereira, A., Paulino, G.H., Menezes, I.F.M., \& Carvalho, M.S. 2014. Polygonal finite elements for incompressible fluid flow. International Journal for Numerical Methods in Fluids, 74(2), 134-151.

VTT subproject (SIMPRO). Computational methods in mechanical engineering product development. url: http://www.vtt.fi/sites/SIMPRO/ vtt-subproject. Acessed in: January, 2018.

van Dijk, N.P., Langelaar, M., \& van Keulen, F. 2014. Element deformation scaling for robust geometrically nonlinear analyses in topology optimization. Structural and Multidisciplinary Optimization, 50(4), 537-560. 
Wallin, M., Ivarsson, N., \& Tortorelli, D. 2018. Stiffness optimization of non-linear elastic structures. Computer Methods in Applied Mechanics and Engineering, 330, 292-307.

Wang, F., Lazarov, B.S., \& Sigmund, O. 2011. On projection methods, convergence and robust formulations in topology optimization. Structural and Multidisciplinary Optimization, 43(6), 767-784.

Wang, F., Lazarov, B.S., Sigmund, O., \& Jensen, J.S. 2014. Interpolation scheme for fictitious domain techniques and topology optimization of finite strain elastic problems. Computer Methods in Applied Mechanics and Engineering, 276, 453-472.

Yang, Y.B., \& Kuo, S.R. 1994. Theory and analysis of nonlinear framed structures. Englewood Cliffs, NJ: Prentice Hall PTR.

Yang, Y.B., \& Shieh, M.S. 1990. Solution method for nonlinear problems with multiple critical points. AIAA journal, 28(12), 2110-2116.

Yoon, G.H., \& Kim, Y.Y. 2005a. Element connectivity parameterization for topology optimization of geometrically nonlinear structures. International Journal of Solids and Structures, 42(7), 1983-2009.

Yoon, G.H., Noh, J.Y., \& Kim, Y. 2011. Topology optimization of geometrically nonlinear structures tracing given load-displacement curves. Journal of Mechanics of Materials and Structures, 6(1), 605-625.

Zhou, M., \& Rozvany, G.I.N. 1991. The COC algorithm, Part II: Topological, geometrical and generalized shape optimization. Computer Methods in Applied Mechanics and Engineering, 89(1-3), 309-336. 


\section{A \\ Verification of the Nonlinear Solution Scheme}

This appendix presents the nonlinear solution method for both Q4 and Q8 elements using the implemented code and the ANSYS ${ }^{\circledR}$ software (APDL, 2017).

The Lee frame model, showed in Figure A.1, is considered for this analysis. The structure exhibits load and displacement limit points which may cause, respectively, the snap-through and snap-back phenomena. Therefore, it is a good model to test the efficiency of the implemented code. The geometry is discretized into 480 elements, two of them in the thickness, so the average size of each element is, approximately, $1 \times 1 \mathrm{~cm}^{2}$ (Parente \& Vaz, 2001).

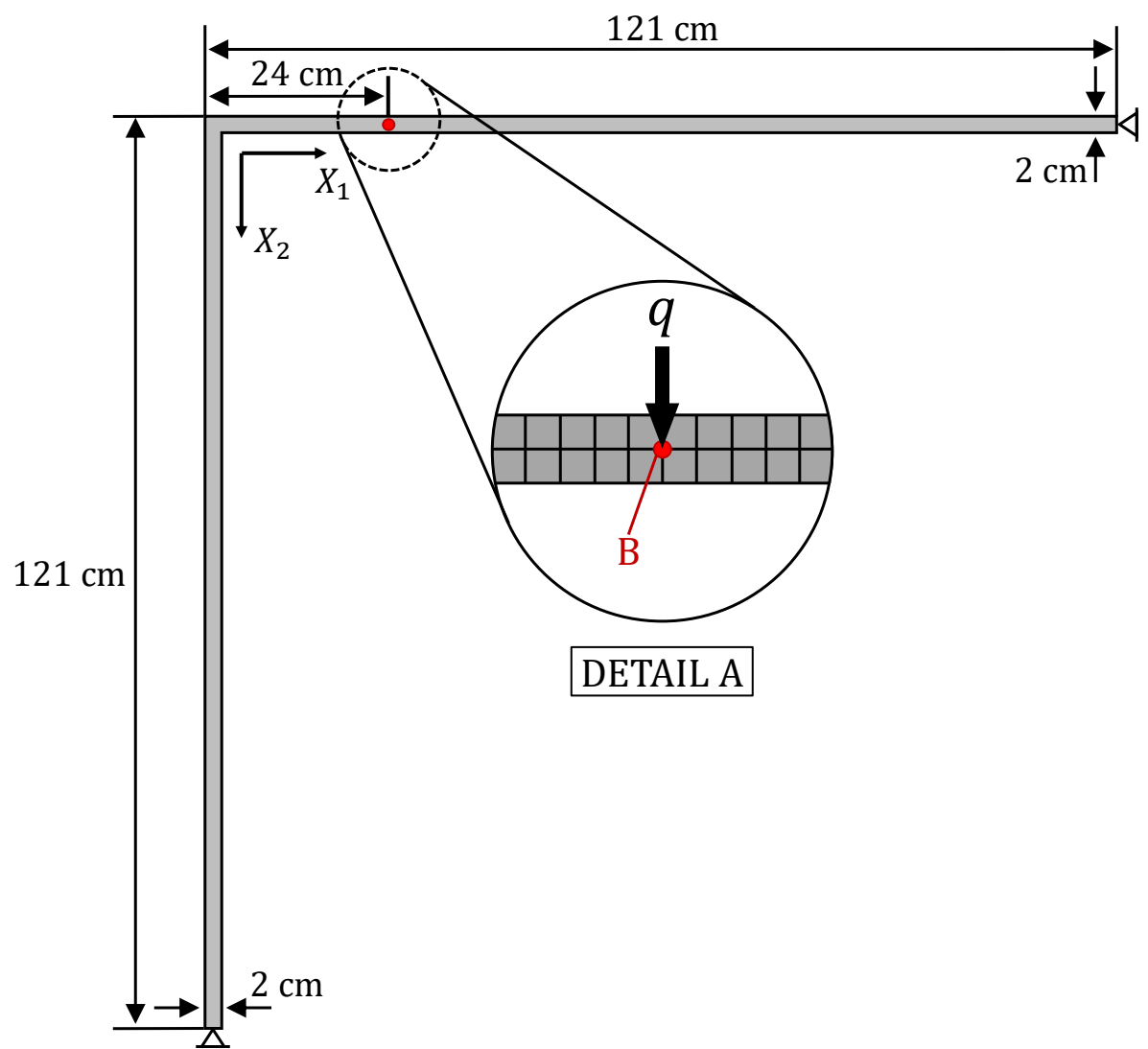

Figure A.1: Lee frame. "DETAIL A" shows the point where the load $\boldsymbol{q}$ is applied (point B). 
The material properties and model characteristics are listed below:

- Material model: SVK

- Configuration: Plane Stress

- Young's modulus $\left(E_{0}\right): 720 \mathrm{kN} / \mathrm{cm}^{2}$

- Poisson's coefficient $\left(\nu_{0}\right): 0.3$

- Depth $(d): 3 \mathrm{~cm}$

- Cross section area $(A): 6 \mathrm{~cm}^{2}$

- Applied load $(q): 2.5 \mathrm{kN}$

The static nonlinear analysis was implemented by means of the Generalized Displacement Control Method (GDCM) and according to the Total Lagrangian finite element formulation. On the other hand, the ANSYS theory is based on the Updated Lagrangian formulation and it allows only the application of the Arc-Length Method (ALM) via its parametric language, APDL (Ansys Parametric Design Language).

Figures A.2 and A.3 show the responses obtained by the developed algorithm and the ANSYS. For Q4 elements, the results are very similar: the absolute difference in the nodal displacements are less than $4 \%$. The response is even better when using Q8 elements in the analysis. In this case, the major difference in nodal displacements is less than 1\%, as observed in the Figure A.3. Figure A.4 illustrates the Lee frame deformed configuration at the points indicated in Figure A.3.

As the results of the proposed algorithm are very close from the ones obtained by the ANSYS $^{\circledR}$ Mechanical $^{\mathrm{TM}}$ APDL, for both types of elements examined, the code is numerically verified. 


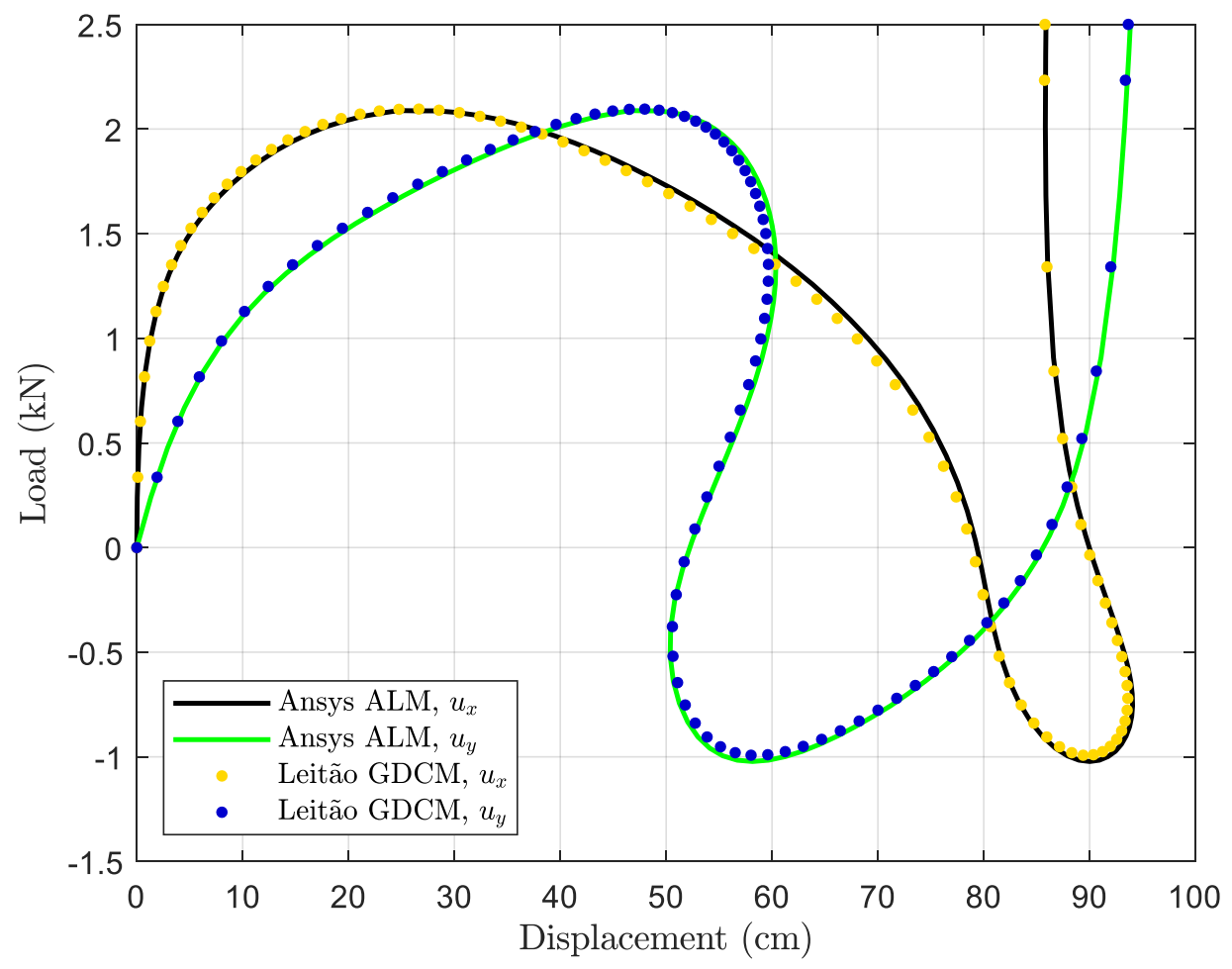

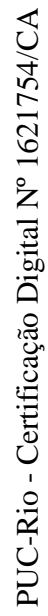

Figure A.2: Lee frame equilibrium path: responses for the implemented code and the APDL for Q4 elements.

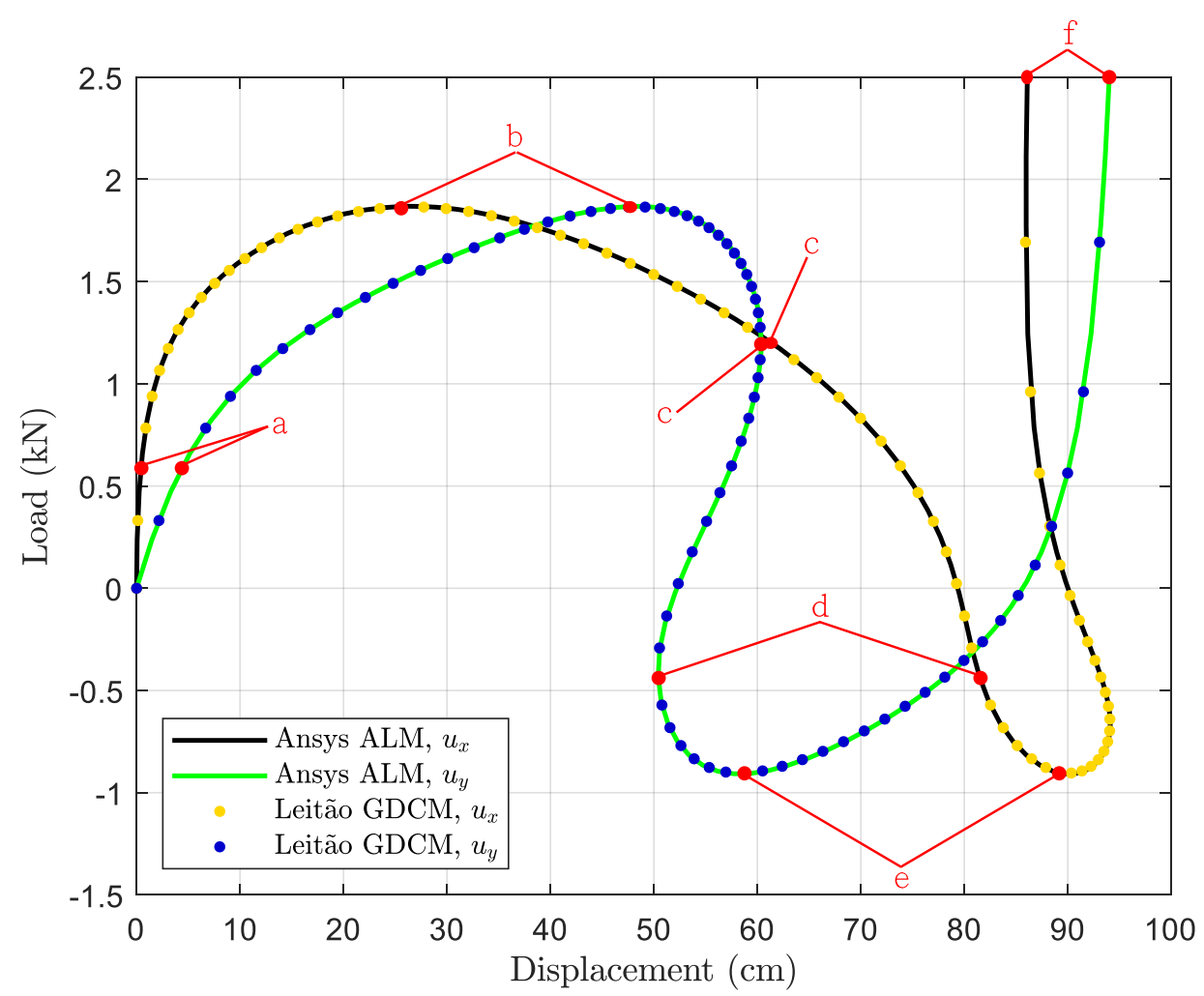

Figure A.3: Lee frame equilibrium path: responses for the implemented code and the APDL for Q8 elements. 


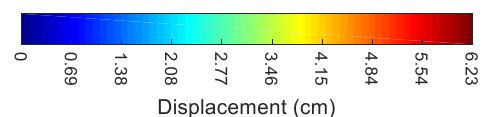

A.4(a): Point a.

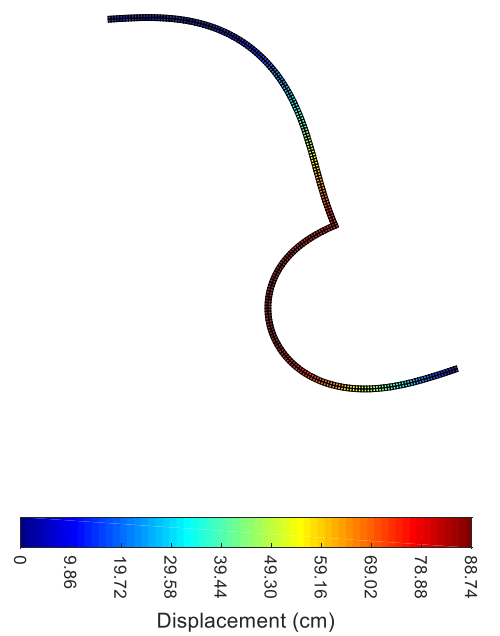

A.4(c): Point c.

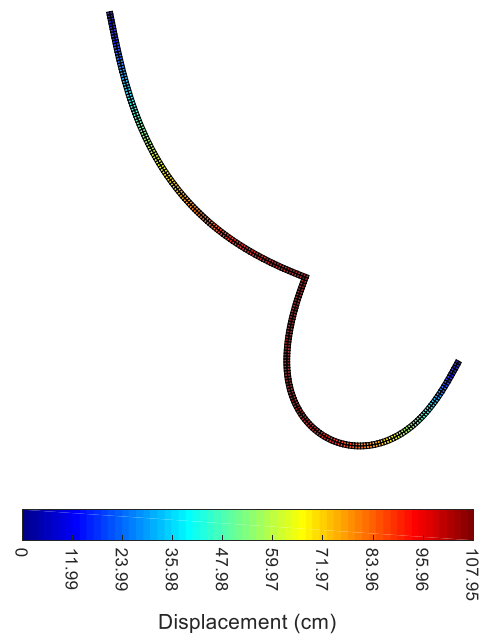

A.4(e): Point e.
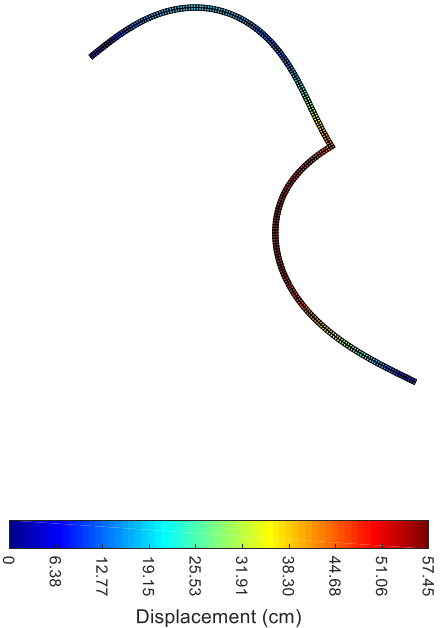

A.4(b): Point b.
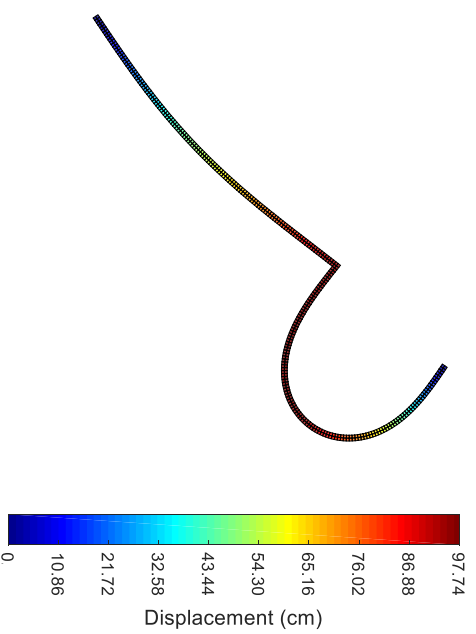

A.4(d): Point d.
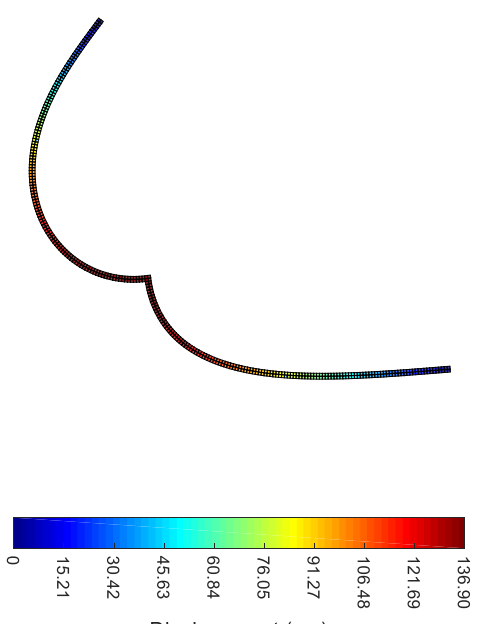

Displacement $(\mathrm{cm})$

A.4(f): Point f.

Figure A.4: Deformation at the points indicated in Figure A.3: (a) linear regime; (b) load limit point in traction; (c) first displacement limit point; (d) second displacement limit point; (e) load limit point in compression; (f) final configuration. 


\section{B \\ Validation of Sensitivity of Objective Function}

In order to verify the accuracy on the objective' sensitivities, the analytical result, computed in Section 4.4, is juxtaposed to the derivatives evaluated by finite difference. The central finite difference is defined by

$$
\frac{d f}{d a} \approx \frac{f(a+\Delta a)-f(a-\Delta a)}{2 \Delta a}
$$

where $\Delta a$ is a small perturbation on the variable $a$. Its value is set to $\Delta a=10^{-8}$. Figure B.1 schematics a fixed-free beam used to evaluate the derivatives. This model has the following properties:

- Material model: SVK

- Configuration: Plane Strain

- Young's modulus $\left(E_{0}\right): 3 \mathrm{GPa}$

- Poisson's coefficient $\left(\nu_{0}\right): 0.4$

- Depth $(d): 1 \mathrm{~m}$

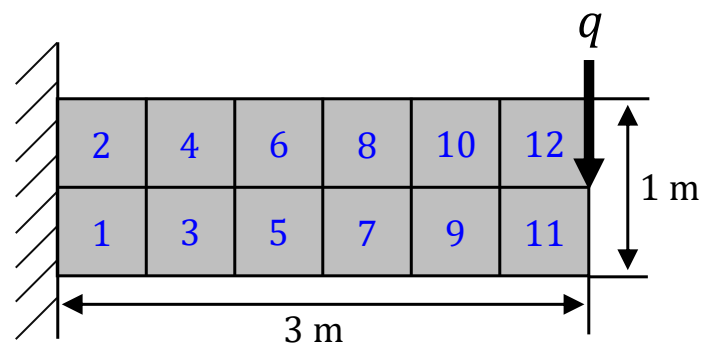

Figure B.1: Tested beam: design domain, boundary conditions, load and element numbering.

The analytical derivatives and those computed via finite differences are compared in Tables B.1 and B.2 for the loads of $15 \mathrm{kN}$ and $100 \mathrm{kN}$, respectively. The relative errors are very small in both cases, which indicates the analytical derivatives are correctly evaluated. 
Table B.1: Comparison between objective function derivatives calculated analytically and via finite differences for a load of $15 \mathrm{kN}$.

\begin{tabular}{c|c|c|c} 
Element No. & Analytical & Finite Difference & Relative Error $(\%)$ \\
\hline 1 & $-14,459.718$ & $-14,459.718$ & $2.735 \cdot 10^{-7}$ \\
\hline 2 & $-14,840.061$ & $-14,840.060$ & $9.892 \cdot 10^{-7}$ \\
\hline 3 & $-12,436.037$ & $-12,436.037$ & $7.938 \cdot 10^{-6}$ \\
\hline 4 & $-13,108.253$ & $-13,108.253$ & $8.901 \cdot 10^{-6}$ \\
\hline 5 & $-7,066.004$ & $-7,066.004$ & $6.768 \cdot 10^{-8}$ \\
\hline 6 & $-3,886.417$ & $-3,886.417$ & $2.161 \cdot 10^{-6}$ \\
\hline 7 & $-5,102.824$ & $-5,102.824$ & $7.337 \cdot 10^{-6}$ \\
\hline 8 & $-2,000.004$ & $-2,000.005$ & $1.603 \cdot 10^{-7}$ \\
\hline 9 & $-12,250.868$ & $-12,250.869$ & $2.460 \cdot 10^{-6}$ \\
\hline 10 & $-2,889.744$ & $-2,889.744$ & $1.665 \cdot 10^{-6}$ \\
\hline 11 & $-1,124.049$ & $-1,124.048$ & $4.712 \cdot 10^{-5}$ \\
\hline 12 & $-1,778.668$ & $-1,778.668$ & $4.574 \cdot 10^{-6}$
\end{tabular}

Table B.2: Comparison between objective function derivatives calculated analytically and via finite differences for a load of $100 \mathrm{kN}$.

\begin{tabular}{c|c|c|c} 
Element No. & Analytical & Finite Difference & Relative Error $(\%)$ \\
\hline 1 & $-422,551.196$ & $-422,551.192$ & $1.018 \cdot 10^{-6}$ \\
\hline 2 & $-496,659.944$ & $-496,659.938$ & $1.248 \cdot 10^{-6}$ \\
\hline 3 & $-336,560.520$ & $-336,560.516$ & $1.233 \cdot 10^{-6}$ \\
\hline 4 & $-454,789.904$ & $-454,789.896$ & $1.633 \cdot 10^{-6}$ \\
\hline 6 & $-206,174.065$ & $-206,174.065$ & $2.627 \cdot 10^{-7}$ \\
\hline 7 & $-393,049.000$ & $-393,048.998$ & $3.714 \cdot 10^{-7}$ \\
\hline 8 & $-231,437.913$ & $-231,437.910$ & $1.346 \cdot 10^{-6}$ \\
\hline 9 & $-415,542.719$ & $-415,542.722$ & $5.785 \cdot 10^{-7}$ \\
\hline 10 & $-328,693.269$ & $-328,693.260$ & $2.766 \cdot 10^{-6}$ \\
\hline 11 & $-452,584.229$ & $-452,584.226$ & $6.221 \cdot 10^{-7}$ \\
\hline 12 & $-379,898.474$ & $-379,898.466$ & $2.141 \cdot 10^{-6}$ \\
\hline
\end{tabular}




\section{C \\ Articles from this Thesis}

This thesis resulted in two papers (Figures C.1 and C.2): one published in the X National Congress of Mechanical Engineering (Leitão \& Pereira, 2018) and another accepted at the 25th Brazilian Congress of Mechanical Engineering (Leitão \& Pereira, 2019).

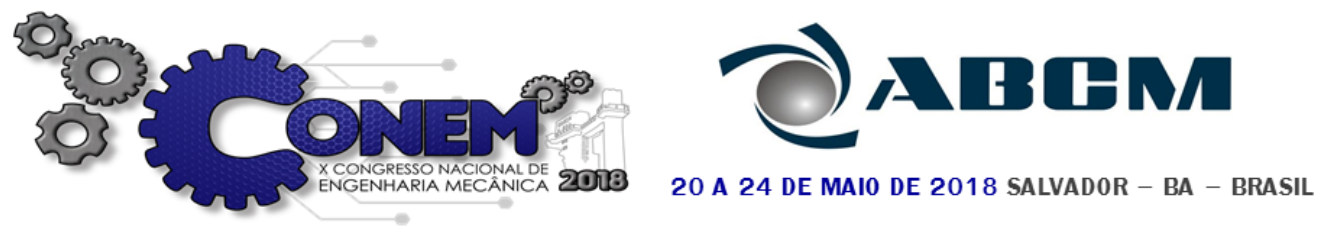

\section{ENERGY INTERPOLATION SCHEME FOR TOPOLOGY OPTIMIZATION OF NONLINEAR ELASTIC STRUCTURES}

André Xavier Leitão, andrex.leitao@aluno.puc-rio.br ${ }^{1}$

Anderson Pereira, anderson @ puc-rio.br ${ }^{1}$

${ }^{1}$ Pontifical Catholic University of Rio de Janeiro, Marquês de São Vicente Street, 225, Gávea - Rio de Janeiro, RJ. Zip code: 22451-900 - PO. Box: 38097

Abstract: Most topology optimization applications in elasticity problems are limited to linear elastic materials and small deformations. However, there are many problems which depict large displacements and a nonlinear theory is required. For loads which magnitudes are within the nonlinear regime, the final shape changes significantly when the optimization considers the nonlinear behavior. The present article aims to study an energy interpolation scheme based on elastic strain function to alleviate the convergence issues related to excessive distortions in void elements. The sensitivity analysis is calculated using the adjoint method. The generalized displacement control method (GDCM) is used to determine the equilibrium points at each step of the nonlinear solution, including the so-called limit points where the Newton-Raphson method fails. Accuracy and applicability of the interpolation scheme are demonstrated by means of a benchmark topology optimization example. The obtained results are in agreement with those in literature and show that the nonlinear theory plays an important role in the optimum design of structural systems, so they must be taken into account when the structure is subject to nonlinear regime.

Keywords: topology optimization, geometric nonlinear analysis, energy interpolation, finite elements, adjoint method.

Figure C.1: Article published in the X CONEM: title, abstract and keywords. 


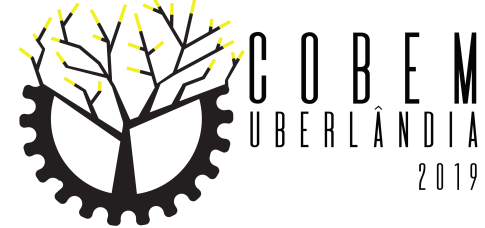

$25^{\text {th }} \mathrm{ABCM}$ International Congress of Mechanical Engineering October 20-25, 2019, Uberlândia, MG, Brazil

\section{COB-2019-1580 \\ TOPOLOGY OPTIMIZATION OF GEOMETRICALLY NONLINEAR STRUCTURES CONSIDERING AN ENERGY INTERPOLATION SCHEME}

\section{André Xavier Leitão}

Anderson Pereira

Pontifical Catholic University of Rio de Janeiro, Marquês de São Vicente Street, 225, Gávea - Rio de Janeiro, RJ. Zip code: 22451-900 andrex.leitao@aluno.puc-rio.br

anderson@puc-rio.br

Abstract. Topology optimization of geometrically nonlinear structures based on the finite element method suffers from numerical instabilities. This is caused by excessive distortions in low-density regions within the design domain which can jeopardize or even result in non-convergence of the optimization process. In this article, an energy interpolation scheme is studied to alleviate this convergence issue. The optimization is solved by the Method of Moving Asymptotes, that requires information on the gradients. These derivatives are computed employing the adjoint method. The NewtonRaphson approach is applied to determine the equilibrium points at each step of the solution during the optimization routine. Comparisons between two different nonlinear solution techniques are exhibited in the scope of the topology optimization method. Applicability and efficiency of this interpolation scheme are demonstrated by means of benchmark structures. The investigated energy interpolation scheme plays a crucial role in the geometric nonlinear analysis, allowing the optimization routine to converge and obtain the optimal material arrangement.

Keywords: Topology Optimization, Geometric Nonlinearity, Finite Elements, Energy Interpolation Scheme, Low-Density Elements

Figure C.2: Article accepted at the 25th COBEM: title, abstract and keywords. 